\title{
A UNIFIED TREATMENT OF TRANSFINITE CONSTRUCTIONS FOR FREE ALGEBRAS, FREE MONOIDS, COLIMITS, ASSOCIATED SHEAVES, AND SO ON
}

\author{
G.M. KELLY
}

Many problems lead to the consideration of "algebras", given by an object $A$ of a category $A$ together with "actions" $T_{k} A \rightarrow A$ on $A$ of one or more endofunctors of $A$, subjected to equational axioms. Such problems include those of free monads and free monoids, of cocompleteness in categories of monads and of monoids, of orthogonal subcategories (= generalized sheaf-categories), of categories of continuous functors, and so on; apart from problems involving the algebras for their own sake.

Desirable properties of the category of algebras - existence of free ones, cocompleteness, existence of adjoints to algebraic functors - all follow if this category can be proved reflective in some well-behaved category: for which we choose a certain comma-category T/A.

We show that the reflexion exists and is given as the colimit of a simple transfinite sequence, if $A$ is cocomplete and the $T_{k}$ preserve either colimits or unions of suitably-long chains of subobjects.

The article draws heavily on the work of earlier authors, unifies and simplifies this, and extends it to new problems. Moreover the reflectivity in $T / A$ is stronger than any earlier result, and will be applied in forthcoming articles, in an enriched version, to

Received 19 November 1979. 
the study of categories with structure.

\section{Introduction}

Many existence theorems in categorical algebra have been proved by transfinite-induction arguments, either leading directly to the universal object sought, or establishing a solution-set condition permitting the application of Freyd's general adjoint functor theorem.

Thus, in the late 1960s, both Ehresmann and Gabriel used such arguments to prove the reflectivity, among presheaves, of the algebras they were considering - namely functors sending chosen cones to limit-cones. In Gabriel and UImer [8] this becomes the theorem on the reflectivity, in a locally-presentable category $A$, of the full subcategory $K^{\perp}$ given by the objects $A$ which are orthogonal to, or sheaves for, each map $k$ of a small set $K$; in the sense that each $A(k, A)$ is an isomorphism. This was later generalized by Freyd and Kelly [7], both in respect to the conditions on $A$ and the size of $K$.

Again, both Schubert [16] and Barr [2], at about the same time and independently, gave such arguments for the existence of colimits in the category of algebras for a monad: under suitable conditions, those of Barr being the weaker. In the same paper, Barr proved under similar conditions the existence of a free monad on a given endofunctor; and later Dubuc did the same for free monoids in any monoidal category - but only under stronger conditions like those of Schubert. Much more recently, Bousfield [5] used such arguments to show, under strong conditions, that a prefactorization system is a factorization system.

Freyd and Kelly remarked in [7] that "Our results seem to bear some relation, not too well understood, to those of Barr and Schubert". The connexion was made clear by Wolff [17], who showed that $\stackrel{\perp}{K}$ consisted of the algebras for a pointed endofunctor $T$ on $A$; since Barr had constructed the free monad on an endofunctor $H$ by constructing the free $H$-algebra, which is also the free $T$-algebra for the pointed endofunctor $T=1+H$, the problems were now closely related.

This was pointed out by the present author in [9], along with a 
further unification and generalization: namely, the category T-Alg of algebras for $T$, whether $T$ is a monad or merely a pointed endofunctor, is a full subcategory of the well-behaved comma category $T / A$; and the desired results all follow by proving it reflective. This reflectivity moreover played an essential role in the thesis of Blackwell [4], who was dealing with a 2-monad $T$ and comparing the strict and lax morphisms of its algebras.

However it still remained true that there were two different kinds of argument for the reflectivity: one in the strong conditions of Gabriel and UImer, Schubert, or Dubuc, which gave the reflexion directly as the colimit of a transfinite sequence, and one in the weaker conditons of Barr or Freyd and Kelly, which used a transfinite argument to get a solution set.

In the meantime, the question of algebras for an endofunctor, especially in its relation to automata theory, had attracted considerable attention from the Prague school; their advances in particular aspects of the question are summed up in Reiterman [15] and Adámek [1], where further references can be found. More recently, Koubek and Reiterman [13] have shown that, even under the weaker type of conditions, the free algebras for an endofunctor $H$ are obtained directly by the convergence of the appropriate transfinite sequence, with no necessity for an appeal to the general adjoint functor theorem. They do not consider pointed endofunctors, although they do give a modification covering the algebras for a monad. Nor do they use the setting of [9] embedding the algebras into $T / A$; instead they use an embedding into "generalized partial $H$-algebras", which would not answer the needs of Blackwell's argument.

These results are now spread over an enormous body of literature, many of the articles in fact being written in ignorance of one another. There is also a scattering of mistakes: not in the statements of major theorems, but rather in incomplete or false proofs. It seems to the present author an appropriate time to write a unified and simplified account, that may serve as a fairly full reference; and at the same time to complete the above work in some important respects. Besides the "constructive" existence in the pointed-endofunctor case, and the reflectivity in $T / A$ which will be used by Blackwell and the author in a forthcoming paper, there are important aspects of the existence of free monoids, and of colimits in categories of monoids, which do not seem to have been 
discussed, but which we include in the present account.

In arranging the account we obtain a conceptual simplification by the following observation. The algebras for a well-pointed endofunctor on $A$ - by which we mean a pointed endofunctor $\sigma: I \rightarrow S$ satisfying $S \sigma=\sigma S$ form a full subcategory of $A$; and in this case the transfinite construction leading to the free algebras, and hence to the reflexion into the algebras, is particularly simple. Then, for a general pointed $T$ (which may be $1+H$ for an unpointed $H$ ), there is a well-pointed endofunctor $S$ on $T / A$ whose algebras form precisely the full subcategory $T$-Alg whose reflectivity we seek; and $S$ is as well-behaved as $T$.

Our desire for completeness, involving us in the discussion of special cases and of counter-examples, has made the technical first chapter a little long; the reader may wish to skim through this and start with Chapter II.

\section{BASIC TECHNICAL RESULTS}

\section{Factorization systems}

1.1. We identify cardinals with initial ordinals, and an ordinal with the set of lower ones. We suppose there is an inaccessible cardinal $\infty$, and call cardinals and ordinals less than $\infty$ small; in future when we say simply " $\alpha$ is an ordinal", we mean a small one. A set is small if its cardinal is small; a category $A$ is small if its set of maps is small, and has small hom-sets if each $A(A, B)$ is small. It is cocomplete if it admits all small colimits. The results are easily adapted to any other view of the foundations.

1.2. By a factorization system $(E, M)$ on $A$ we mean one in the sense of Freyd and Kelly [7], where most of the important properties of such systems are given: one property we use often is that any pushout of an $E$ is an $E$. Another important property, noted by Bousfield [5], only special cases of which are given in [7], is the following: for a natural transformation $\alpha: T \rightarrow S: K \rightarrow A$, if each $\alpha K \in E$, then colim $\alpha: \operatorname{colim} T \rightarrow \operatorname{colim} S$ lies in $E$, whenever these colimits exist. In future we shall write " $\alpha \in E$ " for "each $\alpha K \in E$ ", using the same letters $(E, M)$ for the factorization system on the functor-category 
$[K, A]$ induced pointwise from the system $(E, M)$ on $A$.

Recall that the factorization system $(E, M)$ is said to be proper if every $E$ is an epimorphism and every $M$ a monomorphism; and that this is not required in general. Recall that among the proper ones there are two extreme ones given by (extremal epimorphisms, all monomorphisms) and by (all epimorphisms, extremal monomorphisms), at least if $A$ is a cocomplete category admitting all cointersections of epimorphisms.

1.3. It follows from [7] that every fibred coproduct of maps in $E$ is again in $E$. It is not possible that all such fibred coproducts, of whatever size, should exist, unless every $E$ is an epimorphism. For let $f \in E$, let its cokernel-pair be $u, v$, let $w$ be the unique map with $w u=w v=I$, and let $e$ be the idempotent $v w$. Then $u, v \in E$ as pushouts of $f, w \in E$ since $u$ and $w u$ do, and hence $e \in E$. To prove $f$ epimorphic is to prove $e=1$; suppose the contrary. For some cardinal $\theta$, let the fibred coproduct of $\left(e_{i}: A \rightarrow A_{i}\right)_{i \in \theta}$, where each $A_{i}$ is $A$ and each $e_{i}$ is $e$, be $\left(g_{i}: A_{i} \rightarrow B\right)_{i \in \theta}$. Then any family $h=\left(h_{i}: A_{i}+A\right)_{2 \in \theta}$, where each $h_{i}$ is 1 or $e$, has the form $h_{i}=k g_{i}$ for a unique $k: B \rightarrow A$. Since there are $2^{\theta}$ such families, we have $\operatorname{card} A(B, A) \geq 2^{\theta}$, which is a contradiction if $\theta$ is as big as the cardinal of the set of maps of $A$.

Nor is it possible for those $E$ with a given domain always to form a small set (to within isomorphism), if $A$ is cocomplete and has small homsets, unless every $E$ is an epimorphism; for then in the above argument, since $g_{i} \in E, B$ is one of a small set $\left\{B_{j}\right\}$ of objects depending only on $A$, and we get the contradiction already for a small $\theta$, namely $\max _{j}$ card $A\left(B_{j}, A\right)$. This was first pointed out by Adámek [1].

We call the factorization system $(E, M)$ cocomplete if all fibred coproducts of maps in $E$ do exist; and we now make a blanket assumption to hold throughout this paper (except in an occasional definition or remark where it is clearly irrelevant): the category $A$ is cocomplete, and so is any factorization system $(E, M)$ or $\left(E^{\prime}, M^{\prime}\right)$ on $A$ that we actually use (as distinct from those we merely discuss in $\$ 11$ ). So every $E$ will be an epimorphism; however $(E, M)$ need not be proper, an important case being 
(isomorphisms, all maps).

The E-quotients of an object $A$ (that is, the isomorphism-classes of maps in $E$ with domain $A$ ) then form an ordered set which is a complete lattice (with $I_{A}$ as its greatest element). There is now no distinction between the fibred coproduct of a family of such quotients and that of the corresponding set; it is appropriately called their cointersection. We say that $A$ is E-cowellpowered if each object has only a small set of such quotients; this is by far the most usual case in practice.

1.4. A (not necessarily small, but possibly empty) family of maps $\left(f_{i}: A \rightarrow B_{i}\right)$ is jointly in $M$, or simply in $M$, if given any $p: C \rightarrow D$ in $E$, and any maps $u: A \rightarrow C$ and $v_{i}: B_{i} \rightarrow D$ such that $v_{i} p=f_{i} u$ for all $i$, there is a "unique diagonal fill-in" $t: D \rightarrow A$ with $t p=u$ and $f_{i} t=v_{i}(c f .[7], p .177)$. In our case, since $p$ is epimorphic, we only need some $t$ with $t p=u$; the other commutativity and the uniqueness are then automatic. Since we are supposing pushouts to exist, we can simplify the criterion still further, to that in the following proposition: whose easy proof we leave to the reader.

PROPOSITION 1.1. (i) $\left(f_{i}: A \rightarrow B_{i}\right)$ is in $M$ if and only if, whenever each $f_{i}$ factorizes through $t: A \rightarrow C$ with $t \in E$, then $t$ is an isomorphism.

(ii) If $\left(f_{i}: A \rightarrow B_{i}\right)$ is in $M$ and each $f_{i}$ factorizes through $t: A \rightarrow C$, then $t \in M$.

(iii) If $\left(f_{i}: A \rightarrow B_{i}\right)_{i \in I}$ is in $M$ so is any bigger family $\left(f_{i}: A \rightarrow B_{i}\right)_{i \in J}$ with $J \supset I$.

(iv) If $\left(f_{i}: A \rightarrow B_{i}\right)$ is in $M$ and each $\left(g_{i j}: B_{i} \rightarrow C_{i j}\right)$ is in $M$ then the family $\left(g_{i j} f_{i}: A \rightarrow c_{i j}\right)$ is in $M$.

PROPOSITION 1.2. Any family $\left(g_{i}: D \rightarrow B_{i}\right)$ factorizes voniquely as some $p: D \rightarrow A$ in $E$ followed by a family $\left(f_{i}: A \rightarrow B_{i}\right)$ in $M$.

Proof. Let $\left\{q_{j}\right\}$ be the totality of maps in $E$, with domain $A$, 
through which each $g_{i}$ factorizes, and let $p$ be their cointersection. Then each $g_{i}$ factorizes through $p$ as $f_{i} p$, say; and $\left(f_{i}\right)$ is in $M$ since there is no further $E$ through which all the $f_{i}$ factorize. The uniqueness is clear from the "unique diagonal fill-in".

REMARK. Even without the cocompleteness of A, it is easy to see that the truth of Proposition 1.2 implies, conversely, the cocompleteness of $(E, M)$.

REMARK. When the $g_{i}$ in Proposition 1.2 are in $E, p$ is their union in the lattice of quotient-objects of $D$. This is more familiar in the dual case of $M$-subobjects, when every $M$ is a monomorphism, in which it was introduced in [7], and in which it will be used, for small families, in $\S 2.2$ below.

1.5. The following result is well known in the case of a proper factorization, at least in the cowellpowered case. It use in the present context was suggested by Barr [3].

If $B$ is a full subcategory of $A$, define its $M$-closure to consist of those $A \in A$ admitting some family $\left(f_{i}: A \rightarrow B_{i}\right)$ in $M$ with each $B_{i} \in B$. By Proposition I.I (iii), it comes to the same thing to say that the family $(A, B)$, consisting of alz $f: A+B$ with $B \in B$, is in $M$. Say that $B$ is $M$-closed if it is equal to its $M$-closure; by Proposition 1.1 (iv), the M-closure of $B$ is M-closed. Say that a reflective full subcategory of $A$ is E-reflective if the reflecting maps are in $E$.

PROPOSITION 1.3. A full subcategory $B$ of $A$ is M-closed if and only if it is replete and E-reflective. Then $B$ is itself cocomplete, and $(E, M)$ restricts to a cocomplete factorization system on $B$.

Proof. Let $B$ be E-reflective, with reflexion $K A: A \rightarrow K A$, and let $A$ belong to the M-closure of $E$. Then the family $(A, B)$ factorizes through $K A$, which is therefore in $M$ by Proposition 1.1 (ii), since $(A, B)$ is in $M$. Since $K A$ belongs to $E$, it is an isomorphism, so that $A \in B$ if $B$ is replete.

Conversely, if $B$ is M-closed, and we factorize the family $(A, B)$ as in Proposition 1.2, it is immediate that the $E$-part $K A: A \rightarrow K A$ of the factorization is a reflexion of $A$ into $B$. 
The factorization system restricts to $B$ in the sense that, if a map. $B \rightarrow C$ in $B$ has the factorization $B \rightarrow D \rightarrow C$, then $D \in B$; for $B$ is $M-c l o s e d$ and $D \rightarrow C$ is in $M$.

\section{The effect of endofunctors on cones and colimits}

2.1. A diagram (that is, a functor) $X: K \rightarrow A$ will be called an $\alpha$-chain if the category $K$ is the (small) ordinal $\alpha$, and will be called a sequence if $K=\infty$. For a general $K$ we shall write $X_{B}$ for the value of $X$ on objects, and $X_{\phi}: X_{\beta} \rightarrow X_{\gamma}$ for the value on maps; but mostly we deal with chains or sequences, and then we write the connecting maps as $X_{\beta}^{\gamma}: X_{\beta} \rightarrow X_{\gamma}$ for $\beta \leq \gamma$. We say that a sequence $X$ converges if the $X_{\beta}^{\gamma}$ are isomorphisms for $\gamma \geq \beta \geq$ some $\delta$.

An inductive cone $r=\left(r_{\beta}: X_{\beta} \rightarrow N\right)$ over $X$, with vertex $N$ and generators $r_{\beta}$, may be called a $k$-cone, or an $\alpha$-cone when $k=\alpha$. It factorizes through the colimit-cone $q$ as

$$
r_{\beta}: X_{\beta} \underset{q_{B}}{\longrightarrow} \operatorname{colim} X \underset{\bar{r}}{\longrightarrow} N \text {, }
$$

where in our applications $\bar{r}$ is in fact colim $r$, since $K$ is always at least connected, indeed filtered.

For a factorization-system $(E, M)$ satisfying our blanket assumptions, we call $r$ an $M$-cone, or an $(M, K)$-cone, if each $r_{\beta} \in M$. This implies (because the $E$ are epimorphisms - cf. Proposition 1.I (ii) or [7], Proposition 2.1.4) that each $q_{\beta} \in M$; which we express by saying that $X$ is an $(M, K)$-diagram, or an $(M, \alpha)$-chain. This in turn implies that the connecting maps $X_{\phi}$ are in $M$, but is in general stronger (although not when $A=$ Set,$K$ is filtered, and $M$ is the monomorphisms).

2.2. We say that the cone $r$ is E-tight, or just tight when $E$ is understood, if $\bar{r} \in E$ in (2.1); thus when $E$ is the isomorphisms, the only tight cones are the colimit-cones. In general, if we take the $(E, M)$-factorization of $r_{\beta}$ to be

$$
r_{B}: X_{B} \underset{g_{\beta}}{\longrightarrow} Z_{B} \underset{j_{B}}{\longrightarrow} \text {, }
$$


and make 2 into a functor such that $g$ is natural and $j$ is a cone (using the naturality of the factorization), we have $\bar{r}=\bar{j} \cdot \operatorname{colim} g$. Since colim $g \in E$ because $g \in E$, we see that $r$ is tight if and only if the $M$-cone $j$ is tight. If $(E, M)$ is proper, to say that $j$ is tight is just to say that $N$ is the union of the $j_{\beta}$ in the lattice of M-subobjects; or that $N=U$ im $r_{\beta}$ ( $c f$. the remark at the end of $\$ 1.4$ ).

When $M$ is the monomorphisms and $K$ is filtered, an $M$-cone $j$ has $\bar{j} \in M$ if $A=$ Set; hence also if $A$ is Top or Haus (the categories of topological or hausdorff spaces); hence too if $A$ is locally presentable, provided that now $K$ is sufficiently-highly filtered. In such cases an E-tight $M$-cone is a colimit-cone. But this is no longer true for Top or Haus if $M$ is the subspace-inclusions; for an uncountable power of the reals is not a $k$-space, and is hence the (filtered) union of its compact subspaces without being their colimit. And in general nothing of the kind is true: in the category Comp of compact hausdorff spaces, with $E=$ the epimorphisms = the extremal epimorphisms and $M=$ the monomorphisms = the extremal monomorphisms, the one-point compactification of the natural numbers is the union of the $\omega$-chain of subspaces $\{0,1, \ldots, n\}$, but not their colimit, which is the stone-Čech compactification.

2.3. The most general "smallness condition" we are going to consider on an endofunctor $T: A \rightarrow A$ is:

$$
T \text { preserves the E-tightness of }\left(M^{\prime}, K\right) \text {-cones. }
$$

Here, in accordance with our blanket assumptions, ( $E, M)$ and ( $\left.E^{\prime}, M^{\prime}\right)$ are to be two cocomplete factorization systems on the cocomplete category $A$. What we mean by $(*)$ is that, whenever $r=\left(r_{B}: X_{B} \rightarrow N\right)$ is an $\left(M^{\prime}, K\right)$-cone that is E-tight, then the cone $\operatorname{Tr}=\left(T r_{\beta}: T X_{\beta} \rightarrow T N\right)$ is also E-tight, although it need not in general be an $M^{\prime}$-cone.

We may impose this condition for a single $K$, or for a set of such. The only cases we in fact consider are the following, wherein $\alpha$ is a regular cardinal:

(i) (*) is imposed for $K=\alpha$ alone;

(ii) (*) is imposed when $K$ is any a-filtered (small) 
ordinal $B$;

(iii) (*) is imposed when $K$ is any $\alpha$-filtered small category. Of these, (i) is the weakest (at least a priori, but also in fact: see $\$ 2.6$ below); but is sufficient for our purposes so long as we consider a single $T$. If we have a family $\left(T_{i}\right)$ of endofunctors, we may want each to satisfy ( $i i)$ for some $\alpha_{i}$, so that they simultaneously satisfy (i) for any $\alpha>$ each $\alpha_{i}$. In fact we very often have (iii) if we have anything at all; and although we do not need it here, it is very important in universal algebra.

Special cases of (*) have special names. When $E$ is the isomorphisms, (*) is the condition that $T$ preserves the colimits of all $\left(M^{\prime}, K\right)$-diagrams, in the sense that the canonical comparison map $\tilde{T}$ : colim $T X+T$ colim $X$ is an isomorphism. When $E^{\prime}$ is the isomorphisms, so that $M^{\prime}$ is all maps, it is the condition that $T$ preserves the E-tightness of all K-cones. When both $E$ and $E^{\prime}$ are the isomorphisms, it is that $T$ preserves the colimits of all K-diagrams; when this is so for all $\alpha$-filtered (small) $K$, it is common to say that $T$ has rank less than or equal to $\alpha$ (or loosely that $T$ has rank $\alpha$ ). When $(E, M)$ and $\left(E^{\prime}, M^{\prime}\right)$ coincide and are proper, it is the condition that, whenever $N$ is a $K$-indexed union of M-subobjects $N=U r_{B}$, we have $T N=U$ im $T r_{\beta}$; and if further $T M \subset M$, so that $T r_{\beta}=$ im $T r_{B}$, it is the condition that $T$ preserves $K$-indexed unions.

2.4. Since colimits commute with colimits, and since a colimit of maps in $E$ is in $E$, it follows that a colimit of $E$-tight cones is E-tight. We conclude that if $T: I \rightarrow[A, A]$ is a functor such that each $T_{i}: A \rightarrow A$ satisfies (*), and such that colim $T$ exists, then colim $T: A \rightarrow A$ satisfies (*).

Again, if $\phi: T \rightarrow T^{\prime}: A \rightarrow A$ is in $E$, and if $T$ satisfies (*), so does $T^{\prime}$; for colim $\phi X \in E$ and $\phi N \in E$, so that colim $T^{\prime} r \in E$ if colim $\operatorname{Tr} \in E$.

What is not true is that, if $T, T_{1}: A \rightarrow A$ both satisfy (*), so does $T_{1} T$; unless perchance $T M^{\prime} \subset M^{\prime}$. In some contexts (as when we are 
considering algebras for a monad, and need to look at both $T$ and $T^{2}$ ), we can get around this difficulty by such a trick as that in Remark 4.3 below. But where, as in considerations such as those of $\$ 27.2$ below, we really do need closure under composition, we must in general impose (*) with $M^{\prime}$ equal to all maps, to ensure that $T M^{\prime} \subset M^{\prime}$.

2.5. This case of $M^{\prime}$ equaling all maps is a very special one; it cannot happen except in the important special case where we have $T E \subset E$.

For, $K$ being connected in our applications, we can take for $X$ the constant diagram at any object, whereupon $(*)$ gives $(t)$. Conversely, of course, once we have $(t)$, we get $(*)$ for any $\left(M^{\prime}, K\right)$-cone $r$ once we have it for each colimit $\left(M^{\prime}, K^{\prime}\right)$-cone. Moreover we have:

PROPOSITION 2.1. If $T E \subset E$, the following are equivalent:

(i) $T$ preserves the E-tightness of all K-cones;

(ii) $T$ preserves the E-tightness of all $(M, K)$-cones.

Proof. Given (ii) let $r$ be an E-tight $K$-cone and let it factorize as (2.2). Since $r$ is tight so is $j$, and hence $T j$ by (ii). On the other hand, $T g \in E$ since $g \in E$, so that colim $T g \in E$. It follows that $\operatorname{Tr}$ is tight.

2.6. Because the case $A=$ Set is both extremely special and extremely important, we consider it separately. Here we take $(E, M)$ to be (epimorphisms, monomorphisms). Since every epimorphism is a retraction (we assume the axiom of choice), $(t)$ is satisfied by every endomorphism $T$. Now we have (generalizing Reiterman [15], \$5):

PROPOSITION 2.2. If $K$ is a fiztered ordered set and $T$ is an endofunctor of Set, the following are equivalent:

(i) $T$ preserves the colimits of all K-diagrams;

(ii) $T$ preserves the colimits of all ( $M, K)$-diagrams;

(iii) $T$ preserves the E-tightness of alz (M, K)-cones;

(iv) $T$ preserves thc E-tightness of all K-cones.

Proof. (i) implies (ii) trivially, (ii) implies (iii) since $T E \subset E$, by the remark before Proposition 2.1, and (iii) implies (iv) by 
that proposition. It remains to show that (iv) implies (i).

Let $q_{B}: X_{B} \rightarrow \operatorname{colim} X$ be the colimit of a $K$-diagram. By (iv), the canonical comparison $\tilde{T}: \operatorname{colim} T X \rightarrow T \operatorname{colim} X$ is in $E$, and we succeed if we prove that it is in $M$. Let $x, y \in \operatorname{colim} T X$ with $\tilde{T} x=\tilde{T} y$. Remembering how filtered colimits in Set are constructed, let $x, y$ have representatives $u, v$ in some $T X_{\gamma}$; then $T q_{\gamma} \cdot u=T q_{\gamma} \cdot v$. Since the result is trivial if every $X_{\beta}$ is the empty set 0 , we may suppose that $X_{\gamma} \neq 0$

Let $q_{\gamma}$ have the $(E, M)$-factorization $q_{\gamma}=j p$, let $i$ be a right inverse for the epimorphism $p$, and write $e$ for the idempotent endomorphism ip of $X_{\gamma}$. For each $\beta \geq \gamma$ let $k_{\beta}: X_{\beta} \subset X_{\gamma}$ be the equalizer of $x_{\gamma}^{\beta}$ and $x_{\gamma}^{\beta}$; then $K_{\beta} \subset K_{\beta^{\prime}}$ for $\beta \leq \beta^{\prime}$, and $X_{\gamma}=\underset{\beta \geq \gamma}{U} K_{B}$, since $p e=p$ and hence $q_{\gamma} e=q_{\gamma}$. By setting $K_{\beta}=K_{\gamma}$ if $\beta \neq \gamma$, we can make $K$ into a diagram defined on all of $K$. Applying ( $i v)$ to this diagram gives $T X_{\gamma}=\underset{\beta \geq \gamma}{U} i m k_{\beta}$. So for some $\beta \geq \gamma$ we have $u=T k_{\beta} \cdot u^{\prime}$ and $v=T k_{\beta} \cdot v^{\prime}$. Since $x_{\gamma}^{\beta} k_{\beta}=x_{\gamma}^{\beta} e k_{\beta}$ we conclude that $T X_{\gamma}^{\beta} \cdot u=T X_{\gamma}^{\beta} \cdot T e \cdot u$, and similarly for $v$.

Since $X_{\gamma} \neq 0$ the domain of the monomorphism $j$ is not 0 ; so $j$ is a coretraction and $T j$ is a monomorphism. From $T q_{\gamma} \cdot u=T q_{\gamma} \cdot v$ we therefore get $T p \cdot u=T p \cdot v$ and hence $T e \cdot u=T e \cdot v$. The conclusion of the last paragraph now gives $T X_{\gamma}^{\beta} \cdot u=T X_{\gamma}^{\beta} \cdot v$; so that $x=y$, proving $\tilde{T}$ monomorphic.

A functor $A \rightarrow$ Set is often called small (or petty) if it satisfies the solution-set condition, or equivalently if it is the quotient of a small coproduct of representables. The small endofunctors of Set are precisely those that have some rank a (cf. [15], pp. 69, 70):

PROPOSITION 2.3. For $T$ : Set $\rightarrow$ Set and a regular cardinal $\alpha$, the following are equivalent: 
(i) $T$ preserves the colimits of all a-filtered diagrams;

(ii) $T$ preserves the colimits of all $\alpha$-filtered M-diagrams;

(iii) $T$ is the left Kan extension of its restriction to the full subcategory $S_{\alpha}$ of Set given by the sets of cardinal less than $\alpha$;

(iv) $T$ is a quotient of a small coproduct $\sum \operatorname{Set}\left(B_{\lambda},-\right)$ of representables with card $B_{\lambda}<\alpha$.

Proof. (i) implies (ii) trivially. To see that (ii) implies (iii), let the counit of the Kan extension be

$$
\varepsilon: \int^{B \in S_{\alpha}} T B \times \operatorname{Set}(B,-)+T .
$$

Any set $A$ is the a-filtered M-colimit of its subsets $A_{i}$ of cardinal less than $\alpha$, and both the domain and the codomain of $\varepsilon$ preserve this colimit; since each $\varepsilon A_{i}$ is an isomorphism by Yoneda, so is $\varepsilon A$. (iii) implies $(i)$ since $\operatorname{Set}(B,-)$ preserves $\alpha$-filtered colimits for card $B<\alpha$, and (iii) implies (iv) trivially. On the other hand (iv) implies that each $\varepsilon A$ is at any rate an epimorphism, since it is an isomorphism when $T$ is replaced by $\sum \operatorname{Set}\left(B_{\lambda},-\right)$. To conclude that (iv) implies ( $i$ ii) it suffices therefore to observe that each $\varepsilon A$ is a monomorphism. If elements in the domain of $\varepsilon A$ represented by $(x \in T B, f: B \rightarrow A)$ and by $(y \in T C, g: C \rightarrow A)$ have the same image in $T A$, so that $T f \cdot x=T g \cdot y$, let $i: D \rightarrow A$ be the inclusion of a subset of cardinal less than $\alpha$ containing the images of $f$ and $g$, and not empty unless $A$ is empty. Then $T i$ is a monomorphism since $i$ is a coretraction, so that $T f^{\prime} \cdot x=T g^{\prime} \cdot y$ where $f=i f^{\prime}$ and $g=i g^{\prime}$. Thus $(x, f)$ and $(y, g)$ represent the same element of the domain of $\varepsilon A$.

Reiterman [15] ( $c f$. also Barr [2]) shows that an endofunctor of Set preserving all colimits of w-chains may fail to be small, if and only if there exists a large set of strongly measurable cardinals; with a corresponding result for other regular cardinals $\alpha$ in place of $\omega$.

2.7. Although our interest is in endofunctors, not functors, an 
endofunctor of $A$ may arise as the composite of $T: A \rightarrow A_{1}$ and $F: A_{1} \rightarrow A$, where $E$ (usually a left adjoint) preserves colimits and satisfies $F E_{1} \subset E$, where $\left(E_{1}, M_{1}\right)$ is a suitable factorization system on $A_{1}$. Then for $E T$ to have the property $(*)$ it suffices that $T$ have it, in the sense of sending every $E$-tight $\left(M^{\prime}, K\right)$-cone to an $E_{1}$-tight cone. The remarks of $\$ \S 2.4,2.5$ extend at once to this case.

Note in particular that, when $A_{1}=$ Set and $E_{1}$ is the epimorphisms, and when $T M^{\prime}$ ' is contained in the monomorphisms (as it is when $T$ has the form $A(A,-)$ and $M^{\prime}$ is contained in the monomorphisms), $T$ has the property (*) if and only if it sends each $E$-tight $M^{\prime}$-cone to a colimitcone: by the remarks in $\$ 2.2$.

\section{Examples and counterexamples}

3.1. In spite of the results about special cases in $\$ 2.5$ and $\$ 2.6$, the truth of condition (*) is in general highly sensitive to the factorization systems $(E, M)$ and $\left(E^{\prime}, M^{\prime}\right)$. As far as the dependence on $\left(E^{\prime}, M^{\prime}\right)$ goes, the condition $\left(^{*}\right)$ is weaker the smaller $M^{\prime}$ is, so that we get the strongest results by taking the smallest possible $M^{\prime}$ consistently with our requirements that $\left(E^{\prime}, M^{\prime}\right)$ be cocomplete, or with the stronger requirement of $E^{\prime}$-cowellpoweredness where we need it. Since every $E^{\prime}$ has to be an epimorphism, the smallest possible $M^{\prime}$ is the extremal monomorphisms; and this will suit whenever, as is usually the case, $A$ is epi-cowellpowered. Of course where we need ( $*$ ) to be preserved under composition, we are forced back to $M^{\prime}=$ all maps, as we said in $\$ 2.4$.

In general $\left(^{*}\right)$ is strictly weaker for a smaller $M^{\prime}$. This is of course true in one trivial sense: if $A$ is an $\alpha$-generated but not $\alpha$-presentable object in a locally-presentable category $A$, the functor $\mathrm{A}(A,-): \mathrm{A} \rightarrow$ Set preserves $\alpha$-filtered colimits of mono-diagrams but not of all diagrams; yet in this case $A$ is $B$-presentable for some larger cardinal $B$. We mean rather that, when $M^{\prime}$ is enlarged, (*) may cease to be true for any $\alpha$.

3.2. For instance, consider $T:$ Top $\rightarrow$ Top given by 
$T=\operatorname{Top}(A,-) \cdot B$, where $S \cdot B$ for $S \in$ Set denotes the coproduct of $S$ copies of $B \in$ Top. For $\alpha>\operatorname{card} A$, the functor $\operatorname{Top}(A,-)$ : Top $\rightarrow$ Set sends $\alpha$-filtered unions of subobjects to unions, and hence to colimits by $\S 2.7$; so for $M^{\prime}$ equals the subobject - inclusions equals the extremal monomorphisms, $T$ satisfies $(*)$ for $\alpha$-filtered $K$ both for $E$ equal to the isomorphisms and for any proper $(E, M)$. Yet if we enlarge $M^{\prime}$ to the monomorphisms, and take $A$ to be the chaotic two-point space and $B$ the one-point space $1, \operatorname{Top}(A,-)$ no longer preserves for any regular $\alpha$ the unions of $\left(M^{\prime}, \alpha\right)$-chains, even of those that are colimits. The example ([7], Example 3.3.3) is the a-chain $X$ where each $X_{B}$ is $\alpha+1$ as a set, the connecting maps are identities, and the open sets in $X_{\beta}$ are the empty set, the whole set, and the sets $\{\delta \mid \delta \geq \gamma\}$ for $\gamma \geq \beta$. It follows that $T$ no longer satisfies (*) for any of the above $E$, with $K$ equal to any $\alpha$.

3.3. Again, for the same reasons, the endofunctor $T=\operatorname{Haus}(A,-) \cdot B$ of Haus satisfies (*) for $\alpha$-filtered $K$ with $\alpha>\operatorname{card} A$ if $M^{\prime}$ equals the subspace-inclusions (and a fortiori if $M^{\prime}=$ the extremal monomorphisms $=$ the closed-subspace inclusions), both for $E=$ the isomorphisms and for any $E$ lying between the quotient maps and the surjections. In the special case $A=1$, this remains true (for filtered $K$ ) even for $M^{\prime}=$ the monomorphisms; for the colimits in Haus of monodiagrams are the colimits in Top. Yet when $A=B=1, T$ does not satisfy (*) for $K=$ any $\alpha$, if $E=$ the isomorphisms and $M^{\prime}=$ all maps; even if all the connecting-maps in the $\alpha$-chain are monomorphisms.

For consider the $\alpha$-chain $X$ in which every $X_{\beta}$, as a set, is the disjoint union of two copies of the ordinal $\omega \alpha+1$; the elements corresponding to $\gamma \leq w \alpha$ in the two copies being denoted by $\gamma^{\prime}$ and $\gamma^{\prime \prime}$. Give $X_{B}$ the topology in which each $\left\{\gamma^{\prime}\right\}$ and $\left\{\gamma^{\prime \prime}\right\}$ is open for $\gamma<\omega \alpha$, while a set containing $(\omega \alpha)^{\prime}$ is open if it contains all but a finite number of the $\gamma^{\prime}$ with $\gamma<\omega \beta$, and all the $\gamma^{\prime \prime}$ with $\gamma<\omega \alpha$ from some point on; and symmetrically for $(\omega \alpha)^{\prime \prime}$. With identities for the connecting maps, each $X_{B}$ is hausdorfe but their colimit in Top is not; so that $T$ does not preserve their colimit in Haus.

3.4. On the other hand, for a given $M^{\prime}$, even for $M^{\prime}=$ the extremal 
monomorphisms or $M^{\prime}=$ all maps, the strength of the condition (*) does not vary monotonically with changes in $E$. Thus with $M^{\prime}=$ all maps, the endofunctor $\operatorname{Hom}\left(Z_{2},-\right)$ of abelian groups satisfies (*) for filtered $K$ and $E=$ the isomorphisms, but not for $K=$ any $\alpha$ if $E=$ the epimorphisms. On the other hand the Stone-Čech compactification $T:$ Haus $\rightarrow$ Haus satisfies (*) for any $K$ with $M^{\prime}=$ all maps, if $E=$ the epimorphisms (by $\$ 2.4$, since there is an epimorphism $1 \rightarrow T$ ); yet for any $E$ contained in the surjections it fails for $K=$ any $\alpha$, even if $M^{\prime}$ is changed to the extremal monomorphisms. To see this, take $X_{\beta}$ to be $\beta+1$ with the order-topology, for $\beta<\alpha$, so that colim $X$ is $\alpha$ with the order topology. Since $X_{\beta}$ is compact, the canonical map $\tilde{T}:$ colim $T X \rightarrow T$ colim $X$ is just the inclusion of $\alpha$ in $T \alpha$, which is a subspace inclusion since $\alpha$ is completely regular, but not a surjection since $\alpha$ is not compact.

3.5. Again, the endofunctor $T=\operatorname{Haus}(1,-) \cdot B$ of Haus satisfies (*) for any $K$ with $M^{\prime}=$ all maps, if $E$ is between the quotient maps and the surjections: for $\operatorname{Haus}(1,-)$ sends colimit-cones to epi-tight cones and sends surjections to epimorphisms. We have seen in $\$ 3.3$ that this fails, for $K=$ any $\alpha$, if $E$ is the isomorphisms; it also fails, again for $K=$ any $\alpha$, if $E$ is the epimorphisms, even if now $M^{\prime}=$ the extremal monomorphisms. The example ([7], Example 3.3.3) is the $\alpha$-chain $X$ of $\$ 3.4$ above, with the epi-tight cone given by the embedding of $X_{\beta}=\beta+1$ into $\alpha+1$, the latter again with the order topology.

3.6. In $\$ 10$ below we have to consider an endofunctor $T$, knowing only that it is a regular-epimorphic quotient of $1+A(A,-) \cdot B$. If, say, $A=$ Haus, we can still conclude, by $\$ 3.3$ and $\$ 2.4$, that $T$ satisfies (*) for $\alpha$-filtered $K$ with $\alpha>$ card $A$, if $M^{\prime}$ is the subobjects and $E$ is between the quotient maps and the surjections; but we can no longer conclude it for $E=$ the isomorphisms.

3.7. Barr [2] considers the example where $T$ is the loop-space endofunctor of the category $A$ of pointed hausdorff $k$-spaces, showing that $T$ satisfies $(*)$ for $M^{\prime}=$ subspace-inclusions and $E=$ the isomorphisms, if $K=\omega$. He remarks that his proof, using a result of Dold and Thom, works for no other $K$ than $\omega$. Yet his purpose - to show 
cocompleteness of the category of algebras for the corresponding monad can now equally well be served (see $\$ 25.1$ below) by having $T$ satisfy (*) for $M=M^{\prime}=$ subspace-inclusions and for $K$ sufficiently highly filtered; and this may be easily shown to be so.

In fact $\mathrm{A}(1,-) T=\mathrm{A}(S,-): \mathrm{A} \rightarrow$ Set, where $S$ is the circle. Since $A(S,-)$ preserves the unions of $\alpha$-filtered $M$-cones for $\alpha>\operatorname{card} S$, so does $T$.

\section{A reduction in the well-copowered case}

The trick is to get things to work even though we have $(*)$ only for $\left(M^{\prime}, K\right)$-cones and not all K-cones. One way, suggested by Barr [3] in the case $E=$ the isomorphisms, is to use Proposition 1.2 (with $M^{\prime}$ for its $M$ ). Another is to use the proposition below, due to Koubek and Reiterman ([13], \$8.5, Lemma). This is stronger: not only does it give constructive existence in the applications, but it is the only method the author knows of which establishes cocompleteness of the algebras for a monad when $E=$ the isomorphisms and $M^{\prime}$ is not all maps. On the other hand it requires $E^{\prime}$-cowellpoweredness rather than just cocompleteness of $\left(E ', M^{\prime}\right)$; but then this is a very mild restriction in practice.

If ()$^{\prime}: \infty \rightarrow \infty$ is a strictly-increasing map (= injective functor), and $X$ is any sequence, we write $X^{\prime}$ for the sequence given by $X_{B}^{\prime}=X_{\beta}$. In particular we write $X^{+}$for the sequence given by $X_{B}^{+}=X_{B+1}$; we have the natural transformation $\phi: X \rightarrow X^{+}$with components $\phi_{B}=X_{B}^{\beta+1}$. A cocontinuous strictly-increasing ()$^{\prime}: \infty \rightarrow \infty$ has $0^{\prime}=0$ and $\alpha^{\prime}=\sup _{\beta<\alpha} \beta^{\prime}$ for a limit-ordinal $\alpha$, and is hence defined inductively by giving for each $\beta$ some $(\beta+1)^{\prime}>\beta^{\prime}$.

PROPOSITION 4.1. Let $A$ be $E^{\prime}$-cowezzpowered and $X: \infty \rightarrow A$ any sequence. Then there are a cocontinuous strictly-increasing map $(1): \infty \rightarrow \infty$, a sequence $Y: \infty \rightarrow A$, and a natural transformation $i: Y \rightarrow X^{\prime+}$, such that

(i) $X_{(\beta+1)}^{Y}, i_{\beta} \in M^{\prime}$ for alz $\gamma \geq(\beta+1)^{\prime}$,

(ii) for any $S: A \rightarrow A$ and any limit-ordinal $\alpha$, the map 


$$
\underset{\beta<\alpha}{\operatorname{colim}} S i_{B}: \underset{\beta<\alpha}{\operatorname{colim}} S Y_{\beta} \rightarrow \underset{\beta<\alpha}{\operatorname{colim}} S X(\beta+1)^{\prime}
$$

is an isomorphism.

Proof. We define ( )' inductively; suppose $B^{\prime}$ defined. The $E^{\prime}-$ part of the $\left(E^{\prime}, M^{\prime}\right)$-factorization of $X_{B^{\prime}}^{\gamma}$, for $\gamma \geq B^{\prime}$ gets smaller with increasing $\gamma$, and ultimately becomes stationary by the $E^{\prime}$-cowellpoweredness; take for $(\beta+1)^{\prime}$ the first $\gamma$ at which it achieves this stationary value, and let

$$
\left.X_{\beta}, \underset{f_{\beta}}{\longrightarrow} Y_{\beta} \underset{i_{\beta}}{\longrightarrow} X_{(\beta+1}\right)^{\prime}
$$

be the factorization of $X_{\beta^{\prime}}^{(\beta+1)^{\prime}}$. Using the naturality of the factorization, make $Y$ into a functor in such a way that $f: X^{\prime} \rightarrow Y$, $i: Y \rightarrow X^{\prime+}$ are natural. Then $(i)$ is automatic from the construction.

Consider the diagram

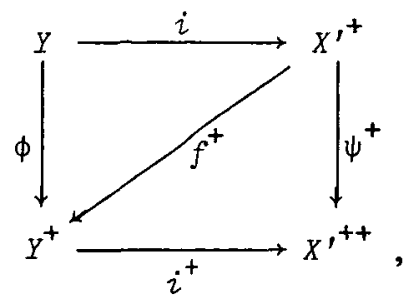

where $\phi_{\beta}=y_{\beta}^{\beta+1}$ and $\psi$ is the corresponding map for $X^{\prime}$. Since (4.1) expresses that $\psi=$ if, the bottom triangle of (4.2) commutes. Since $\phi f=f^{+} \psi$ by the naturality of $f$, we also have by (4.1) that $\phi f=f^{+} i f$, whence $\phi=f^{+} i$ since $f \in E^{\prime}$ is an epimorphism. Thus the top triangle of $(4.2)$ commutes.

Now apply $S$ to $(4.2)$ and pass to the colimit over the segment $\beta<\alpha$. Writing for simplicity colim $S i$ for $\underset{B<\alpha}{\operatorname{colim}} S i_{B}$ and so on, and observing that colim $S Y^{+}=\operatorname{colim} S Y$ with colim $S \phi=1$, and similarly for colim $S \psi^{+}$, we conclude that colim $S f$ and colim $S i$ are inverse, giving $(i i)$.

PROPOSITION 4.2. Let $A$ be E'-cowellpowered, let $S: A \rightarrow A$ satisfy (*) for $K=$ the regular cardinal $\alpha$, and let $X: \infty \rightarrow A$. Then 
there is a limit-ordinal $\alpha^{\prime}$ such that, for each $\gamma \geq \alpha^{\prime}$, if the cone $\left(X_{B}^{\gamma}: X_{B} \rightarrow X_{Y}\right)_{B<\alpha}$, is E-tight for some $(E, M)$, so is the cone $\left(S X_{\beta}^{\gamma}\right)_{\beta<\alpha^{\prime}}$

Proof. Let ()$^{\prime}, Y, i$ be as in Proposition 4.1, and let $\left\{X_{B}^{\gamma}\right\}_{\beta<\alpha^{\prime}}$ be tight (=E-tight). Since the $\beta^{\prime}$ with $\beta<\alpha$ are final in $\alpha^{\prime}$, so that $\left(X_{\beta}\right)_{\beta<\alpha}$, and $\left(X_{\beta},\right)_{\beta<\alpha}$ have the same colimit, the cone $\left(X_{\beta}^{Y},\right)_{\beta<\alpha}$ is also tight; equally the cone $\left(X_{(\beta+I)}^{\gamma} \cdot\right)_{\beta<\alpha}$, and so too the $M^{\prime}$-cone $\left\{X(\beta+1), i_{\beta}^{\gamma}\right)_{B<\alpha}$, since $\underset{\beta<\alpha}{\operatorname{colim}} i_{\beta}$ is an isomorphism.

Since $S$ satisfies $(*), S$ of this last cone is also tight. Now reversing the above steps, since $\underset{\beta<\alpha}{\operatorname{colim}} S i_{\beta}$ is an isomorphism, we have the desired result that $\left(S X_{\beta}^{\gamma}\right]_{\beta<\alpha}$ is tight.

REMARK 4.3. If in Proposition 4.1 we start with two sequences $X, \bar{X}: \infty \rightarrow \mathrm{A}$, we can use the same ( )' for both, getting $i: Y \rightarrow X^{\prime+}$ and $\bar{i}: \bar{Y}+\bar{X}^{+}$with the desired properties; we merely rewrite the proof taking for $(\beta+1)^{\prime}$ the first $\gamma$ at which the $E^{\prime}$-parts of both $X_{\beta}^{Y}$, and $\bar{X}_{\beta}^{\gamma}$, achieve their stationary values.

If, in the proof of Proposition 4.2, we use this improved version of Proposition 4.1, taking $\bar{X}$ to be $S X$, we get an improved version of Proposition 4.2, in which the phrase after the last comma is replaced by "so are the cones $\left(S X_{B}^{\gamma}\right)_{B<\alpha}$, and $\left(S^{2} X_{B}\right)_{B<\alpha}$,".

\section{WELL-POINTED ENDOFUNCTORS}

\section{The free-algebra sequence}

5.1. A pointed endofunctor $(S, \sigma)$ on $A$ is an endofunctor 
$S: \mathrm{A} \rightarrow \mathrm{A}$ together with a natural transformation $\sigma: 1 \rightarrow S$. An $(S, \sigma)-$ algebra $(A, a)$ is an object $A$ of $A$ together with an action $a$ of $(S, \sigma)$ on $A$; by which is meant a map $a: S A \rightarrow A$ satisfying $a \cdot \sigma A=1_{A}$. Where confusion is unlikely we abbreviate $(S, \sigma)$ to $S$ and $(A, a)$ to $A$, and speak of the $S$-algebra $A$. With an S-algebra-map $f:(A, a) \rightarrow\left(A^{\prime}, a^{\prime}\right)$ defined as a map $f: A \rightarrow A^{\prime}$ for which $f \cdot a=a ! \cdot S f$, the $S$-algebras form a category $S-\mathrm{Alg}$. The forgetful functor $U: S-\mathrm{Alg} \rightarrow \mathrm{A}$ sending $(A, a)$ to $A$ clearly creates limits and $U$-absolute colimits; and hence is monadic whenever it admits a left adjoint.

We say that $S=(S, \sigma)$ is a wezl-pointed endofunctor if $S \sigma=\sigma S: S \rightarrow S^{2}$. As we said in the Introduction, we shall first give conditions for the existence of free $S$-algebras in the well-pointed case, where the transfirite construction is particularly simple; and then all our later existence results will be obtained by various reductions to this case. From now to the end of $\S 8$ it is a standing hypothesis, repeated occasionally for emphasis, that $S$ is a well-pointed endofunctor.

LEMMA 5.1. For a welz-pointed $S$ and any map $g: S B \rightarrow A$, if $f: B \rightarrow A$ is the composite $g \cdot \sigma B$, then $S f=\sigma A \cdot g$.

Proof. $\sigma A \cdot g=S g \cdot \sigma S B$ by naturality; but this is $S(g \cdot \sigma B)$ since $S \sigma=\sigma S$.

Recall that a map $h$ in $A$ and an object $A$ are said to be orthogonal if $A(h, A)$ is an isomorphism. Following Wolff [17], we have:

PROPOSITION 5.2. For a well-pointed $(S, \sigma)$ the following properties of $A \in A$ are equivalent:

(i) A admits some S-action a;

(ii) $\sigma A$ is an isomorphism, so that $A$ admits the unique $S$-action $a=(\sigma A)^{-1}$;

(iii) $A$ is orthogonal to $\sigma B$ for each $B \in A$.

Thus S-Alg is isomorphic to, and may be identified with, the full replete subcategory of A determined by such objects; whereupon $U: S-\mathrm{Alg} \rightarrow \mathrm{A}$ is identified with the inclusion. 
Proof. First, $(i) \Rightarrow(i i)$ : since $a \cdot \sigma A=1$, Lemma 5.1 gives $\sigma A \cdot \alpha=S 1=1$. Next, $(i i) \Rightarrow($ iii): to say that $A$ is orthogonal to $\sigma B$ is to say that each $f: B \rightarrow A$ factorizes uniquely through $\sigma B$. But $f=(\sigma A)^{-1} \cdot S f \cdot \sigma B$ by naturality; and if $f=g \cdot \sigma B$, then $g=(\sigma A)^{-1} \cdot S f$ by Lema 5.1. Finally, $(i i i) \Rightarrow(i)$ : since $A$ is orthogonal to $\sigma A$, we have some $a$ with $a \cdot \sigma A=1$.

5.2. Supposing $A$ cocomplete, in accordance with our blanket assumptions, we define inductively a sequence $\hat{S}=\left(S^{B}\right)_{B<\infty}: \infty \rightarrow[A, A]$. We set $S^{0}=1_{A} ; S^{\beta+1}=S S^{\beta}$ with connecting-map $\hat{S}_{B}^{\beta+1}$ equal to $\sigma S^{\beta}: S^{\beta}+S S^{\beta}$; and, for a limit-ordinal $\alpha, S^{\alpha}=\underset{\beta<\alpha}{\operatorname{colim}} S^{\beta}$ with, as connecting-maps $\hat{S}_{\beta}^{\alpha}: S^{\beta} \rightarrow S^{\alpha}$, the generators of the colimit-cone. If, for a given $A \in A$, the map $\sigma S^{\alpha} A: S^{\alpha} A \rightarrow S S^{\alpha} A$ is an isomorphism for some $\alpha$, then $\hat{S}_{\beta}^{\gamma}$ is an isomorphism for $\gamma \geq \beta \geq \alpha$, and the sequence $\hat{S} A=\left(S^{\beta} A\right)$ converges; we may say that it converges at $\alpha$.

PROPOSITION 5.3. If $\hat{S} A$ converges at $\alpha$, then $S^{\alpha} A$ is the free $S$-algebra on $A$, the reflexion of $A$ into $S-A l g$ being $\hat{S}_{0}^{\alpha} A: A \rightarrow S^{\alpha} A$.

Proof. It follows inductively from Proposition 5.2 (iii) that any map from $A$ into an $S$-algebra $B$ factorizes uniquely through each $\hat{S}_{0}^{\beta}$; and $S^{\alpha} A$ is an $S$-algebra by Proposition 5.2 (ii).

We call $\hat{S} A$ the free-algebra sequence for $A$, and when it converges we say that the free algebra on $A$ exists constructively. If this happens for every $A$ (the $\alpha$ at which convergence occurs depending in general on $A$ ) we may say that $S-A l g$ is constructively reflective in A. The idempotent monad on $A$ corresponding to this reflexion is then $S^{\infty}=\operatorname{colim} S^{\beta}$, this large colimit existing pointwise, $S^{\infty} A$ being the $B<\alpha$ ultimate value of $S^{\beta} A$.

REMARK 5.4. S-Alg may be reflective without being constructively 
so. Take for $A$ the cocomplete category $\infty+1$, define $S$ by $S \beta=\beta+1$ for $\beta<\infty, S_{\infty}=\infty$, and take for $\sigma$ the unique map $1 \rightarrow S$. Then $S-A l g=\{\infty\}$ is reflective but not constructively so.

LEMMA 5.5. The sequence $\hat{S}^{+}$, in the sense of $\$ 4$, coincides with the sequence $S \hat{S}$.

Proof. Since $\hat{S}_{\beta}^{\gamma}=\hat{S}_{\beta+1}^{\gamma} \cdot \sigma S^{\beta}$ for $\gamma>\beta$, Lemma 5.1 gives $S \hat{S}_{\beta}^{\gamma}=\sigma S^{\gamma} \cdot \hat{S}_{\beta+1}^{\gamma} ;$ which is $\hat{S}_{\beta+1}^{\gamma+1}$.

\section{The constructive existence theorem}

Theorem 6.2 below is the (slightly generalized) analogue, in the present context, of Theorem 8.5 of Koubek and Reiterman [13].

PROPOSITION 6.1. Let $A$ be E-cowezipowered, let $A \in A$, and zet $\alpha$ be a.limit-ordinal such that, for each $\gamma \geq \alpha$, if the cone $\left(\hat{S}_{\beta}^{\gamma} A: S^{\beta} A+S^{\gamma} A\right)_{B<\alpha}$ is E-tight, so is the cone $\left(S \hat{S}_{\beta}^{\gamma}\right)_{B<\alpha}$. Then $\hat{S} A$ converges.

Proof. We show inductively that the cone $\left(\hat{S}_{\beta}^{\gamma}\right\}_{B<\alpha}$ is tight for all $\gamma \geq \alpha$. When $\gamma=\alpha$, it is tight because it is a colimit-cone. For the passage from $\gamma$ to $\gamma+1$, we use the hypothesis of the proposition, together with Lemma 5.5. For the passage to a limit-ordinal, we use the observation of $\$ 2.4$ that a colimit of tight cones is tight.

Now to say that $\left(\hat{S}_{B}^{\gamma} A\right)_{B<\alpha}$ is tight is to say that $\hat{S}_{\alpha}^{\gamma} A$ is in $E$. Hence $\hat{S} A$ converges by the E-cowellpoweredness.

Combining this with Proposition 4.2 now gives:

THEOREM 6.2. Let $A$ be $E$ - and $E^{\prime}$-cowellpowered, let $S$ be a well-pointed endofunctor, and for some regular cardinal $\alpha$ let $S$ preserve the E-tightness of $\left(M^{\prime}, \alpha\right)$-cones. Then $S-\mathrm{Alg}$ is constructively reflective in $A$.

REMARK 6.3. When both $E$ and $E^{\prime}$ are the isomorphisms, so that $S$ preserves all colimits of $\alpha$-chains, the sequences $\hat{S} A$ all converge at 
$\alpha$. In the more general cases we have no such "uniformity" of the convergence.

\section{7. $\mathrm{An}^{2}$ existence theorem without cowellpoweredness}

We now give the analogue (once again generalized) of the alternative existence proof of Barr's manuscript [3].

PROPOSITION 7.1. Let $(S, \sigma)$ be a well-pointed endofunctor, let $\phi: S \rightarrow S^{*}$ be a map of endofunctors with each $\phi A$ epimorphic, and set $\sigma^{*}=\phi \sigma: 1 \rightarrow S^{*}$. Then $\left(S^{*}, \sigma^{*}\right)$ is a well-pointed endofunctor, and $S^{*}-\mathrm{Alg}$ consists of those $S$-algebras $A$ for which $\phi A$ is an isomorphism.

Proof. By Lemma 5.1, $\sigma S^{*} \cdot \phi=S \sigma^{*}$ since $\sigma^{*}=\phi \sigma$. Hence $\sigma^{*} S^{*} \cdot \phi=\phi S^{*} \cdot \sigma S^{*} \cdot \phi=\phi S^{*} \cdot S \sigma^{*}$, which is $S^{*} \sigma^{*} \cdot \phi$ by naturality. Since $\phi$ is epimorphic, we have $\sigma^{*} S^{*}=S^{*} \sigma^{*}$ as desired.

By Proposition 5.2, $A$ is an $S^{*}$-algebra precisely when $\sigma^{*} A=\phi A \cdot \sigma A$ is invertible. But then $\sigma A$ has a left inverse, so by Proposition 5.2 is already invertible; whence the result.

Now let $(S, \sigma)$ be a well-pointed endofunctor on $A$, and $(E, M)$ a cocomplete factorization system on $A$. Let $B$ be the M-closure in $A$ of $S$-Alg, in the sense of $\$ 1.5$, and let $K: 1 \rightarrow K: A \rightarrow A$ be the E-reflexion of $A$ into $B$ given by Proposition 1.3.

PROPOSITION 7.2. $K S: A \rightarrow A$ with $K \sigma: 1 \rightarrow K S$ is a well-pointed endofunctor on A whose algebras coincide with $S-\mathrm{Alg}$.

Proof. $k \sigma: l \rightarrow K S$ is the composite of $\sigma: 1 \rightarrow S$ and $\kappa S: S \rightarrow K S$. Since the latter is in $E$ and hence an epimorphism, $K S$ is well-pointed by Proposition 7.1. Moreover its algebras are those $S$-algebras $A$ for which $K S A$ is an isomorphism; but this is all $S$-algebras. For if $A$ is an $S$-algebra, $S A \cong A$ is already in $B$.

Write $S^{*}: B \rightarrow B$ for the restriction to $B$ of $K S$, with $\sigma^{*}$ for the restriction of $k \sigma$. Then $S^{*}$ is a well-pointed endofunctor on the cocomplete $B$, the algebras for which again coincide with $S-A l g$. Recall from Proposition 1.3 that $(E, M)$ restricts to a cocomplete factorization system on $B$.

PROPOSITION 7.3. For each $B \in B$, all the connecting maps in the free-algebra sequence $\hat{S}^{*}$ are in $M$; and if $B$ admits a reflexion 
$f: B \rightarrow C$ into $S-A l g$, then $f \in M$. For any $A \in A$, the reflexion of $A$ into $S-\mathrm{Alg}$ exists if the sequence $\hat{S}^{*} K A$ converges; and when $A$ is $M-$ wellpowered, it exists only if this sequence converges.

Proof. The first sentence follows from Proposition 1.1 (ii), since any map from $S^{*} \beta_{B}$ into an $S$-algebra factorizes through each $S_{\beta}^{*} \gamma_{B}$, and since, when the reflexion exists, every map from $B$ into an $S$-algebra factorizes through $f$. In this latter situation $f$ factorizes as $f_{\beta} \cdot \hat{S}_{0}^{*} \beta_{B}$ for each $B$; and $f_{\beta} \in M$, being the reflexion into $S-A l g$ of. $S^{*} B_{B}$; so that $\hat{S}^{*} B$ is a sequence of M-subobjects of $C$, and necessarily converges if $A$ is $M$-wellpowered. The remainder follows from Proposition 5.3 .

PROPOSITION 7.4. A cone $\left(r_{B}: X_{B}+N\right)$ in $B$ is E-tight as a cone in $B$ if and only if it is E-tight as a cone in A.

Proof. If $\operatorname{colim} X$ denotes the colimit of $X$ in $A$, so that its colimit in $B$ is $K$ colim $X$, the induced map $r: \operatorname{colim} X \rightarrow N$ factorizes as $K \operatorname{colim} X: \operatorname{colim} X \rightarrow K \operatorname{colim} X$, followed by $\bar{r}^{*}: K \operatorname{colim} X \rightarrow N$, say. Since $k \in E$ we have $\bar{r}^{*} \in E$ if and only if $\bar{r} \in E$.

THEOREM 7.5. Let $S$ be a well-pointed endofunctor. If, for some regular $\alpha$, either $S$ preserves the E-tightness of $(M, \alpha)$-cones, or else $S$ preserves the colimits of $(M, \alpha)$-chains, the sequence $\hat{S}^{*} B$ converges at $\alpha$ for each $B \in B$, and free algebras exist.

Proof. Under the first hypothesis, by $\$ 2.4$, since $K S: S \rightarrow K S$ is in $E$, the endofunctor $K S$ of $A$ preserves the E-tightness of $(M, \alpha)$-cones since $S$ does. But then, by Proposition 7.4, the endofunctor $S^{*}$ of $B$ preserves the E-tightness of $(M, \alpha)$-cones.

Write $X$ for the sequence $\hat{S}^{*} B$. Then $\left(X_{\beta}^{\alpha}: X_{\beta}+X_{\alpha}\right)_{\beta<\alpha}$ is an M-cone by Proposition 7.3 , and is E-tight since it is a colimit-cone in $B$. Hence by hypothesis $\left(S^{*} X_{B}^{\alpha}\right)_{B<\alpha}$ is E-tight. But by Lemma 5.5 this latter is $\left(x_{\beta+1}^{\alpha+1}\right)_{\beta<\alpha} ;$ its E-tightness means that $x_{\alpha}^{\alpha+1}$, $=\sigma^{*} X_{\alpha}$, lies in $E$; and since it lies in $M$ by Proposition 7.3 , it is an isomorphism; so 
that $X_{\alpha}=S^{*}{ }_{B}$ is an $S$-algebra, and the sequence converges.

Turning to the second hypothesis, we observe that $\left(X_{B}\right)_{B<\alpha}$ is an $(M, \alpha)$-chain in $A$, since $\left(X_{\beta}^{\alpha}\right)_{B<\alpha}$ is an $M$-cone; so that by hypothesis the canonical map

$$
\underset{\beta<\alpha}{\operatorname{colim}} S X_{\beta} \rightarrow \underset{\beta<\alpha}{\operatorname{colim}} X_{\beta}
$$

is an isomorphism, where these are the colimits in $A$. Write $Z$ for colim $X_{\beta}$, with generators $q_{\beta}: X_{B} \rightarrow Z$.

$\beta<\alpha$

Now by Lemma 5.1 , since $\kappa S \cdot \sigma=\sigma^{*}$, the composite

$$
S X_{\beta} \underset{\kappa S X_{B}}{\longrightarrow} K S X_{\beta}=X_{\beta+1} \underset{\sigma X_{B+1}}{\longrightarrow} S X_{\beta+1}
$$

is $S \sigma^{*} X_{\beta}$, $=S X_{\beta}^{\beta+1}$; so that its colimit in $A$ over $\beta<\alpha$ is the identity map of $\underset{\beta<\alpha}{\operatorname{colim}} S X_{\beta}$. Therefore $\underset{\beta<\alpha}{\operatorname{colim} \kappa S X_{\beta}}$ is a coretraction; since it is also in $E$ because $k \in E$, it is an isomorphism; so that the cone

$$
S X_{B} \underset{\kappa S X_{B}}{\longrightarrow} K S X_{B}=X_{B+1} \underset{q_{B+1}}{\longrightarrow} 2
$$

is the colimit over $\beta<\alpha$ of $S X_{\beta}$. It easily follows that (7.1) is just $\sigma Z: Z \rightarrow S Z$; for $\sigma Z \cdot q_{\beta+1} \cdot K S X_{\beta}=S q_{\beta+1} \cdot \sigma X_{\beta+1} \cdot K S X_{B}$ by naturality, and by (7.2) this is $s q_{B+1} \cdot s X_{B}^{\beta+1}=S q_{B}$.

Thus $Z$ is an $S$-algebra, since $\sigma Z$ is an isomorphism. Since $Z$ is therefore already in $B$, it is also the colimit in $B$ of $\left(X_{\beta}\right)_{B<\alpha}$; that is, it is $X_{\alpha}=S^{*^{\alpha}}{ }_{B}$. Hence the sequence converges.

REMARK 7.6. The author cannot see how to adapt this argument to the slightly more general hypothesis, as in Theorem 6.2, that $S$ preserves the E-tightness of $\left(M^{\prime}, \alpha\right)$-cones; however the hypotheses of Theorem 7.2 cover most practical cases.

REMARK 7.7. The fact that free $S^{*}$-algebras exist constructivelv in 
these circumstances by no means implies that free $S$-algebras exist constructively. In the example of Remark 5.4, with $M=$ the extremal monomorphisms, both the hypotheses of Theorem 7.5 are trivially satisfied; and $B$ reduces to $S-a l g=\{\infty\}$.

\section{The case $S E \subset E$}

In the important but special case where the well-pointed $S$ satisfies $S E \subset E$, we can say a certain amount even when $S$ satisfies no global condition of the form $(*)$ of $\$ 2.3$. First:

PROPOSITION 8.1. Let $S E \subset E$. Then if $\sigma A \in E$ for some $A \in A$ the free $S$-algebra on $A$ exists, and is given by the $K A: A \rightarrow K A$ of $\$ 7$. If, moreover, $A$ is E-cowellpowered, the free S-algebra on $A$ exists constructively.

Proof. Since $\sigma K A \cdot K A=S K A \cdot \sigma A$ by naturality, since $\sigma A$ and $K A$ are in $E$, and since $S K A \in E$ because $S E \subset E$, we have $\sigma K A \in E$. But $\sigma K A \in M$ by Proposition 1.1 (ii), since $K A \in B$ and every map from $K A$ to an $S$-algebra factorizes through $\sigma K A$. So $\sigma K A$ is an isomorphism, so that $K A$ is already in $S-A l g$.

For the final assertion we argue inductively that $\hat{S}_{0}^{B} \in E$ for all $B$, whence the sequence $\hat{S} A$ converges by the E-cowellpoweredness. The assertion is trivial for $B=0$, and for $B=1$ it is the hypothesis $\sigma A \in E$. for the passage from $\beta$ to $\beta+1$, $S E \subset E$ gives $S \hat{S}_{0}^{\beta} \in E$, which by Lemma 5.5 says $\hat{S}_{I}^{\beta+1} A \in E$, giving $\hat{S}_{0}^{\beta+1} A \in E$. The closure of $E$ under cointersections gives the passage to a limit-ordinal.

REMARK 8.2. When $\sigma A \in E$ for all $A$, the well-pointedness of $S$ is automatic by Proposition 7.1, and $S E \subset E$ is also automatic. Moreover $S$ preserves the E-tightness of all cones by $\$ 2.4$. Hence Proposition 8.1 is then a special case of Theorems 6.2 and 7.5 .

PROPOSITION 8.3. Let $S E \subset E$, and let $f: A+B$ be in $E$. If the free S-algebra on $A$ exists, so does that on $B$; and if the first exists constructively, so does the second, in the case where $A$ is E-cowelzpowered. 
Proof. Consider the pushout

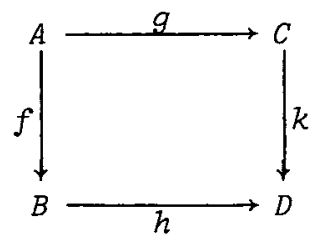

where $g: A \rightarrow C$ is the reflexion of $A$ into $S$-Alg. Then since $f \in E$ we have $k \in E$ and hence $S k \in E$. Since $\sigma C$ is an isomorphism we conclude that $\sigma D \in E$, so that by Proposition 8.1 , $D$ admits a reflexion $t: D \rightarrow E$ into $S-A l g$. Since $g$ is orthogonal to every $S$-algebra, so is its pushout $h$; and since $t$ too is orthogonal to every $S$-algebra, so is th (cf. [7], Proposition 2.1.3). Since $E$ is an S-algebra, it follows that th: $B \rightarrow E$ is the reflexion of $B$ into $S-A I g$.

For the constructive part, we observe that there is a natural transformation $s=\hat{S} f: \hat{S} A+\hat{S} B$, given by $s_{0}=f, s_{\beta+1}=S s_{\beta}$, and $s_{\alpha}=\underset{\beta<\alpha}{\operatorname{colim}} s_{\beta}$ for a limit-ordinal $\alpha$. Since $f \in E, S E \subset E$, and $E$ is closed under colimits, we have $s \in E$. It follows that, if $\hat{S}_{\beta}^{\gamma} A$ is an isomorphism for $\gamma \geq \beta \geq \alpha$, then $\hat{S}_{\beta}^{\gamma} \in E$ for $\gamma \geq \beta \geq \alpha$; whence by the E-cowellpoweredness $\hat{S} B$ converges.

We now consider the situation (which occurs in $\S 9$ and $\$ 16.2$ below) of well-pointed endofunctors $(S, \sigma)$ on $A$ and $\left(S^{\prime}, \sigma^{\prime}\right)$ on $A^{\prime}$, together with a functor $G: A^{\prime} \rightarrow A$ (which commonly has a right adjoint $V: A \rightarrow A^{\prime}$ ), and a natural transformation $\theta: G S^{\prime} \rightarrow S G$ satisfying

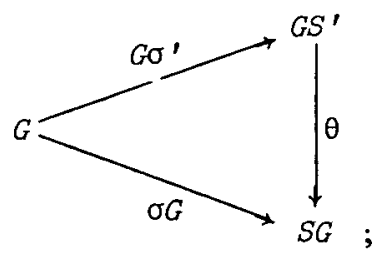

here $A$ and $A$ ' are to be cocomplete, and $A$ is to have a cocomplete factorization system $(E, M)$.

PROPOSITION 8.4. In the situation above, let $S E \subset E$ and let $\theta \in E$. Then if $A$ is $E$-cowellpowered and the free $S^{\prime}$-algebra on 
$B \in A^{\prime}$ exists constructively, so does the free $S$-algebra on $G B \in A$. If $A$ is not necessarily E-cowellpowered, but $G$ has a right adjoint $V$, and the free $S^{\prime}$-algebra on $B$ exists, so does the free $S$-algebra on $G B$.

Proof. For the first part, consider the natural transformation $\hat{\theta}: G \hat{S}^{\prime} \rightarrow \hat{S} G$ whose components $\hat{\theta}_{\beta}: G S^{\prime B}+S^{\beta} G$ are defined inductively as follows: $\hat{\theta}_{0}=1: G \rightarrow G, \hat{\theta}_{B+1}$ is the composite

$$
G S^{\prime} S^{\prime B} \underset{\theta S^{\prime \beta}}{\longrightarrow} S G S^{\prime B} \underset{S \hat{\theta}_{\beta}}{\longrightarrow} S S^{\beta} G \text {, }
$$

and, for a limit-ordinal $\alpha, \hat{\theta}_{\alpha}=\underset{\beta<\alpha}{\operatorname{colim}} \hat{\theta}_{\beta} ;$ the naturality is immediate from (8.1). It follows by induction that each $\hat{\theta}_{\beta} \in E$; so that if $\hat{S}^{\prime} B$ converges so does $\hat{S} G B$, by the latter argument used in the proof of Proposition 8.3 .

For the second part, let $\phi: S^{\prime} V \rightarrow V S$ be the mate of $\theta$ under the adjunction (cf. [10], §2); it satisfies the transform

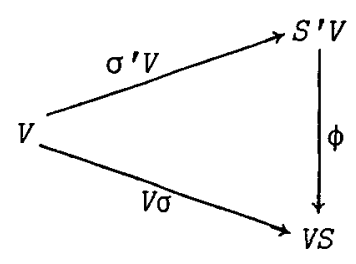

of (8.1). For any $S$-algebra $A, \sigma A$ is invertible; hence by (8.2), $\sigma^{\prime} V A$ has a left inverse; so that $V A$ is an $S^{\prime}$-algebra by Proposition 5.2. Hence $V A$ is orthogonal to the reflexion $f: B \rightarrow C$ of $B$ into $S^{\prime}-\mathrm{Alg}$; whence by the adjunction $A$ is orthogonal to $G f: G B \rightarrow G C$. It follows that the reflexion of $G B$ into $S$-Alg exists if that of $G C$ does; which it does indeed by Proposition 8.1, $\sigma G C$ being in $E$ by (8.1) since $\theta \in E$ and since $\sigma^{\prime} C$ is an isomorphism.

\section{The construction of well-pointed endofunctors}

Recall that a pointed endofunctor $(R, \rho)$ on $A$ is called an idempotent monad if $R^{2}=R$ with $\rho R=R \rho=1$; it is clearly wellpointed, and its algebras are reflective in A. Moreover every full 
replete reflective subcategory of A consists of the algebras for such an idempotent monad $(R, \rho)$, which is uniquely determined to within isomorphism.

There are a number of processes that, starting from well-pointed endofunctors, produce others. The well-pointed endofunctors to which we shall apply the results of this chapter all arise from the repeated application of these processes, starting with idempotent monads.

The first such process is that of Proposition 7.1 above. Another is the following; note that the family it refers to does not have to be small, so long as the fibred coproduct exists - which it will do if $\sigma_{i} \in E$ for all but a small set of $i$.

PROPOSITION 9.1. Let $\left(S_{i}, \sigma_{i}\right)$ be a family of well-pointed endofunctors on $A$ and let $\sigma: I \rightarrow S$ be the fibred coproduct of the family $\sigma_{i}: 1 \rightarrow S_{i}$. Then $(S, \sigma)$ is well-pointed and $S-A l g$ is the intersection of the categories $S_{i}-\mathrm{Alg}$.

Proof. Let the generators of the colimit cone be $\rho_{i}: S_{i} \rightarrow S$. The equation $S_{i} \sigma_{i}=\sigma_{i} S_{i}$, composed with $\rho_{i} \rho_{i}: S_{i} S_{i} \rightarrow S S$, gives the equation $S \sigma \cdot \rho_{i}=\sigma S \cdot \rho_{i}$; this being so for all $i$, we have $S \sigma=\sigma S$. The last statement is clear from Proposition 5.2.

The final (and central) such process is that given by Wolff $([17]$, Theorem 2.1).

PROPOSITION 9.2. Let $G \rightarrow V: A \rightarrow A^{\prime}$ be an adjunction with counit $\varepsilon: G V \rightarrow 1$, let $\left(S^{\prime}, \sigma^{\prime}\right)$ be a well-pointed endofunctor on $A^{\prime}$, and define a pointed endofunctor $(S, \sigma)$ on A by the pushout

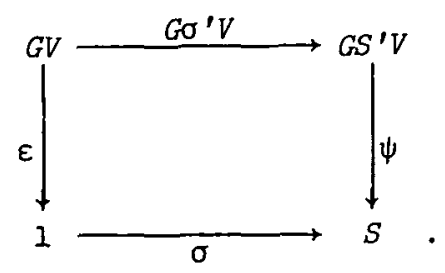

Then $(S, \sigma)$ is well-pointed, and $S-A I_{B}$ consists of those $A \in A$ for which $V A$ is an S'-algebra. Moreover the natural transformations 
$\theta: G S^{\prime} \rightarrow S G$ and $\phi: S^{\prime} V \rightarrow V S$ corresponding to $\psi$ satisfy (8.1) and (8.2).

Proof. The last statement is immediate for $\phi$ and hence follows for $\theta$. Composing the equality $S^{\prime} \sigma^{\prime} V=\sigma^{\prime} S^{\prime} V: S^{\prime} V \rightarrow S^{\prime 2} V$ with $\phi S \cdot S^{\prime} \phi: S^{\prime 2} V \rightarrow V S^{2}$, and simplifying by the use of (8.2), we get $V S \sigma \cdot \phi=V \sigma S \cdot \phi$, or equivalently $S \sigma \cdot \psi=\sigma S \cdot \psi$. Since $S \sigma \cdot \sigma=\sigma S \cdot \sigma$ by naturality, we have $S \sigma=\sigma S$ because (9.1) is a pushout.

The following are successively equivalent: $A$ is an $S$-algebra; $I_{A}$ factorizes through $\sigma A ; \varepsilon A$ factorizes through $G \sigma^{\prime} V A ;{ }_{V A}$ factorizes through $\sigma^{\prime} V A ; V A$ is an $S^{\prime}$-algebra.

REMARK 9.3. The above proposition will most often be used when $\left(S^{\prime}, \sigma^{\prime}\right)$ is an idempotent monad $(R, \rho)$ on $A^{\prime}$.

\section{ORTHOGONAL SUBCATEGORIES AND RELATED QUESTIONS}

\section{Orthogonal subcategories}

This chapter treats some applications where a subcategory to be proved. reflective may be exhibited fairly directly as the category of algebras for a well-pointed endofunctor. The first of these is that mentioned in the Introduction: given a set $K$ of maps in $A$, we consider the full subcategory $K^{\perp}$ of $A$ given by the objects orthogonal to each $k \in K$.

We suppose for this chapter that A has small hom-sets; then for each object $M$ we have the representable functor $A(M,-): A \rightarrow$ Set, with a left adjoint sending $X$ to the coproduct $X \cdot M$ of $X$ copies of $M$. The counit $\eta_{M} A: A(M, A) \cdot M \rightarrow A$ of this adjunction has, for each $f \in A(M, A)$, its $f$-component equal to $f$.

For each map $k: M \rightarrow N$ the functor $V: A \rightarrow \operatorname{Set}^{2}$ sending $A$ to $A(k, A)$ has a left adjoint $G$, whose value $G v$ at $v: X \rightarrow Y$ is given by the pushout 


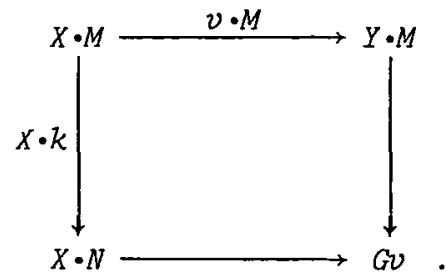

There is an idempotent monad $R$ on $\operatorname{Set}^{2}$ sending $v: X \rightarrow Y$ to $R v=I_{Y}: Y \rightarrow Y$, with unit $\rho v=(v, 1)$; its algebras consist of those $v$ that are isomorphisms; so that $A$ is orthogonal to $k$ if and only if $V A$ is an $R$-algebra.

It follows from Proposition 9.2 that the full subcategory $k^{+}$of $A$ is $S$-Alg, where $(S, \sigma)$ is the well-pointed endofunctor on A given by the pushout $(9.1)$, with $(R, \rho)$ for $\left(S^{\prime}, \sigma^{\prime}\right)$. An easy calculation gives $(S, \sigma)$ explicitly as the pushout

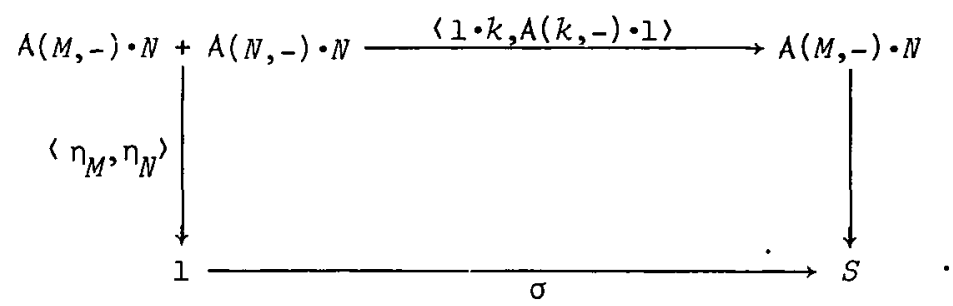

If we now consider a set $K=\left\{k_{i}: M_{i} \rightarrow N_{i}\right\}$ of maps and rewrite (10.1) replacing $k, M, N, \sigma, S$ by $k_{i}, M_{i}, N_{i}, \sigma_{i}, S_{i}$, and then take $(S, \sigma)$ to be the fibred coproduct of the $\left(s_{i}, \sigma_{i}\right)$ as in Proposition 9.1 (if it exists), we conclude that $K^{\perp}=S-\mathrm{Alg}$ for the well-pointed endofunctor $S$.

For a cocomplete factorization system $\left(E^{\prime}, M^{\prime}\right)$ on $A$, consider the "local presentability" property

(P) for each $A \in A$ there is a regular cardinal $\alpha_{A}$ such that $A(A,-): A \rightarrow$ Set preserves the colimits of $\left(M^{\prime}, \beta\right)$-chains for each $\alpha_{A}$-filtered ordinal $B$.

THEOREM 10.1. Let $K$ be small, and for some regular cardinal let each $\mathrm{A}\left(M_{i},-\right)$ and each $\mathrm{A}\left(N_{i},-\right)$ preserve the colimits of 
(M', $\alpha)$-chains; which is the case if A satisfies (P). Then $K^{\perp}$ is reflective, and constructively so if $A$ is $E^{\prime}$-cowezlpowered.

Proof. Each $S_{i}$ preserves by $\$ 2.4$ the colimits of $\left(M^{\prime}, \alpha\right)$-chains, since the other three corners of (10.1) do so and $S_{i}$ is the colimit of that diagram. Hence $S$, as the fibred coproduct of the $\alpha_{i}: 1 \rightarrow S_{i}$, does so too. The result now follows from Theorems 6.2 and 7.5 .

The above is essentially Corollary 3.1 of Wolff [17]; the case $M^{\prime}=$ all maps includes Satz 8.5 of Gabriel and UImer [8] and the remarks in their $\$ \S 8.6$ and 8.7. The smallness of $K$ is used in Theorem 10.1 in two ways: to ensure the existence of the fibred coproduct $S$ - which might be ensured in other ways - and to get from $(P)$ the hypotheses we really use, on $\mathrm{A}\left(M_{i},-\right)$ and $\mathrm{A}\left(N_{i},-\right)$.

The possibility of dealing with certain cases of a large $K$, at the cost of strengthening $(P)$, was first recognized in the (non-constructive) treatment of Freyd and Kelly [7]. Suppose that, for some cocomplete factorization system $(E, M), k_{i}$ has the factorization

$$
M_{i} \underset{k_{i}^{\prime}}{\longrightarrow} L_{i} \underset{k_{i}^{\prime \prime}}{\longrightarrow} N_{i}
$$

Then, because $k_{i}^{\prime}$ is epimorphic, it easily follows (cf. [7], Lemma 4.12) that $k_{i}^{\perp}=k_{i}^{\prime \perp} \cap k_{i}^{\prime \prime}$. Let $\left(S_{i}^{\prime}, \sigma_{i}^{\prime}\right)$ and $\left(S_{i}^{\prime \prime}, \sigma_{i}^{\prime \prime}\right)$ be what we get in (10.1) when we replace $k$ by $k_{i}^{\prime}$ and by $k_{i}^{\prime \prime}$. If we now suppose further that the system $(E, M)$ is proper, we have that $\sigma_{i}^{\prime} \in E$; for it is the pushout of the top edge of (10.1), which is in $E$ since its composite with one of the injeciions is the map $1 \cdot k_{i}^{\prime}$ of $E$ (we are using Proposition 2.1 .4 of [7]). Thus the $\sigma_{i}^{\prime}: I \rightarrow S_{i}^{\prime}$ admit a fibred coproduct $\sigma^{\prime}: 1 \rightarrow S^{\prime}$ with $\sigma^{\prime} \in E$.

To proceed, we must suppose that the number of different $k_{i}^{\prime \prime}$ (to within isomorphism) is small; since $A$ has small hom-sets, this is to say that the numbers of different $L_{i}$ and $N_{i}$ are small; and if $A$ is $M$-wellpowered, it is to say that the number of different $N_{i}$ is small. 
We may then form the fibred coproduct $\sigma^{\prime \prime}: 1 \rightarrow S^{\prime \prime}$, not of the family $\sigma_{i}^{\prime \prime}: 1 \rightarrow S_{i}^{\prime \prime}$, but of the small set of different elements which comprise it. Finally we may form the pushout $\sigma: 1 \rightarrow S$ of $\sigma^{\prime}: 1 \rightarrow S^{\prime}$ and $\sigma^{\prime \prime}: 1 \rightarrow S^{\prime \prime}$; this need not of course coincide with our previous $S$, even when $K$ is small; but it still satisfies $S-\mathrm{Alg}=K^{\perp}$. Moreover we have a map $\phi: S^{\prime \prime} \rightarrow S$ which, as the pushout of $\sigma^{\prime}: 1 \rightarrow S^{\prime}$, is in $E$.

In relation to the above proper $(E, M)$ and some $\left(E^{\prime}, M^{\prime}\right)$ consider, in place of $(P)$, the "local boundedness" property

(B) for each $A \in A$ there is a regular cardinal $\alpha_{A}$ such that, whenever $\beta$ is an $\alpha$-filtered ordinal, $A(A,-): A \rightarrow$ Set sends each $E$-tight $\left(M^{\prime}, B\right)$-cone to an epi-tight cone.

This condition is most often considered for $M^{\prime}=M$, and then asserts ( $c f$. $\S 2.3)$ that $A(A,-)$ preserves $\alpha_{A}$-filtered ordinal unions of $M$-subobjects (in the sense of taking them to ordinary unions in Set). It may be a little weaker than this if $M^{\prime} \subset M$; but there is no point in imposing it for an $M^{\prime}$ bigger than $M$. Since it is thus contemplated only for a proper $\left(E^{\prime}, M^{\prime}\right)$, it is by $\$ 2.7$ stronger than (P) - in general strictly so, by $\$ \S 3.3$ and 3.5. Yet with $M^{\prime}=M$ it is satisfied by $\$ 2.2$ by any locally-presentable $A$ with $M=$ the monomorphisms, and by $\$ \$ 3.2$ and 3.3 by any category like Top or Haus with $M=$ the subspace-inclusions; similarly by any category like topological groups with $M=$ the subgroups that are subspaces.

The following improves in various minor respects - including the dropping of the completeness hypothesis on A - Theorem 4.1.3 of Freyd and Kelly [7].

THEOREM 10.2. For a proper $(E, M)$, with the $k_{i}$ factorizing as in (10.2), let the number of different $k_{i}^{\prime \prime}$ be smalz. For some regular $\alpha$, let each $A\left(L_{i},-\right)$ send $E$-tight $\left(M^{\prime}, \alpha\right)$-cones to epi-tight cones; which is the case if $A$ satisfies (B). Then ${ }^{\perp}$ is constructively reflective when $A$ is $E$ - and $E^{\prime}$-cowellpowered; and is at any rate reflective when $E^{\prime}=E$.

Proof. Since the functor $-\cdot_{i}:$ Set $\rightarrow A$ preserves colimits and 
sends epimorphisms to retractions which surely lie in $E$, the endofunctor $\mathrm{A}\left(L_{i},-\right) \cdot N_{i}$ preserves the E-tightness of $\left(M^{\prime}, \alpha\right)$-cones. Since 1 does too, so by $\$ 2.4$ does $S_{i}^{\prime \prime}$, which by $(10.1)$ is a regular quotient, and hence an E-quotient, of $1+A\left(L_{i},-\right) \cdot N_{i}$. So then, by $\$ 2.4$ again, does the fibred coproduct $S^{\prime \prime}$ of the different $S_{i}^{\prime \prime}$. So again, by $\$ 2.4$ once more, does $S$, as an E-quotient of $S^{\prime \prime}$. The result now follows from Theorems 6.2 and 7.5 .

\section{Factorization systems}

A closely-related problem is that of whether, for a set $K$ of maps in A as above, the pre-factorization system $(F, N)=\left(K^{\downarrow \uparrow}, K^{\downarrow}\right)$, in the notation of Freyd and Kelly [7], §2.1, is actually a factorization system.

We recall from [7] that, for any prefactorization system $(F, N)$, $p: A \rightarrow B$ is in $F$ if and only if, for every commutative diagram

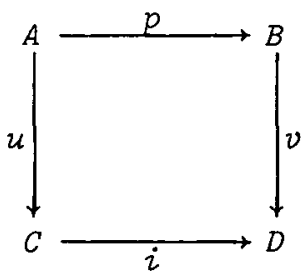

with $i \in N$, there exists a "unique diagonal fill-in" $w: B \rightarrow C$.

LEMMA 11.1. When A admits pullbacks, we may conclude that $p \in F$ if the above "unique diagonal fill-in" property holds for those diagrams (11.1) in which $D=B$ and $v=1$.

Proof. We factorize $p$ and $u$ through the pullback of $i$ and $v$; we leave the reader to complete the easy proof.

For $E \in A$, write $N_{E}$ for the full subcategory of $A / E$ whose objects are those maps in $N$ with codomain $E$.

PROPOSITION 11.2. If the prefactorization system $(F, N)$ is a factorization system, then $N_{E}$ is reflective in $A / E$ for each $E \in A$; and the converse is true if A admits pullbacks.

Proof. The direct assertion is immediate: if $f: A \rightarrow E$ has the 
$(F, N)$-factorization
$(11.2)$
$A \underset{p}{\longrightarrow} B \underset{j}{\longrightarrow} E$,

it is clear that $p: f \rightarrow j$ is the reflexion into $N_{E}$ of $f \in A / E$. For the converse, let (11.2) be this reflexion; we use Lemma 11.1 to show that $p \in F$.

Suppose then that $p=i u$ with $i \in N$ (as in (1l.1) with $D=B$ and $v=1)$. Since $j i \in N_{E}$ the map $u: f \rightarrow j i$ of $A / E$ factorizes uniquely through the reflexion $P$; that is, there is a unique $w: B \rightarrow C$ with $w p=u$ and $j i w=j$. In fact $i w=1$ as desired; for it is a map $j \rightarrow j$ in $N_{E}$, and its composite with the reflexion $p$ is $i w p=i u=p$.

We now proceed by applying Theorems 10.1 and 10.2 to $A / E$ in place of $A$. The category $A / E$ is cocomplete, with colimits formed as in A; each factorization system $(E, M)$ on $A$ induces one, with the same name, on $A / E$; and $A / E$ is $E$-cowellpowered if $A$ is. As for the effect on cones of $(A / E)(a,-)$, where $a: A \rightarrow E$ is an object of $A / E$, consider a B-cone $\left(r_{\gamma}: x_{\gamma} \rightarrow n\right)_{\gamma<\beta}$ in $A / E$, where $x_{\gamma}: X_{\gamma} \rightarrow E$ and $n: N \rightarrow E$; it is clear from the formation of filtered colimits in Set that $(A / E)\left(\alpha, r_{\gamma}\right)$ is a colimit cone or an epi-tight cone if the same is true of $A\left(A, r_{\gamma}\right)$.

Write $K_{E}$ for the set of all maps in $A / E$ of the form $k_{i}: f \rightarrow g$, where $k_{i}: M_{i} \rightarrow N_{i}$ is in $K$ and where $f: M_{i} \rightarrow E, g: N_{i} \rightarrow E$. It is clear that, if $N=K^{\downarrow}$, then $N_{E}$ is the full subcategory $K_{E}^{\perp}$ of $A / E$. Since $A$ has small hom-sets, $K_{E}$ is small if $K$ is; and more generally, if $K$ is such that the number of different $k_{i}^{\prime \prime}$ in (10.2) is small, the corresponding thing is true of $K_{E}$. Thus:

THEOREM 11.3. Let A admit pullbacks. Then in the circumstances of either Theorem 10.1 or Theorem 10.2, $(F, N)=\left(K^{\downarrow \uparrow}, K^{\downarrow}\right)$ is a factorization system on $\mathrm{A}$. 
The requirement that $A$ admit pullbacks, although a very mild restriction in practice, is inelegant when the core of the argument uses only colimits. In the simplest case, corresponding to Theorem 10.1 with cowellpoweredness, we can remove it, and at the same time describe $F$ more concretely.

Write $K^{+}$for the smallest set of maps containing $K$, closed under composition and containing the isomorphisms, closed under pushout, and closed under colimits in the sense of $\$ 1.2$.

LEMMA 11.4. Each component of the $\sigma$ of (10.1) Zies in $k^{+}$.

Proof. The top and bottom disamonds in the following commutative diagram are pushouts:

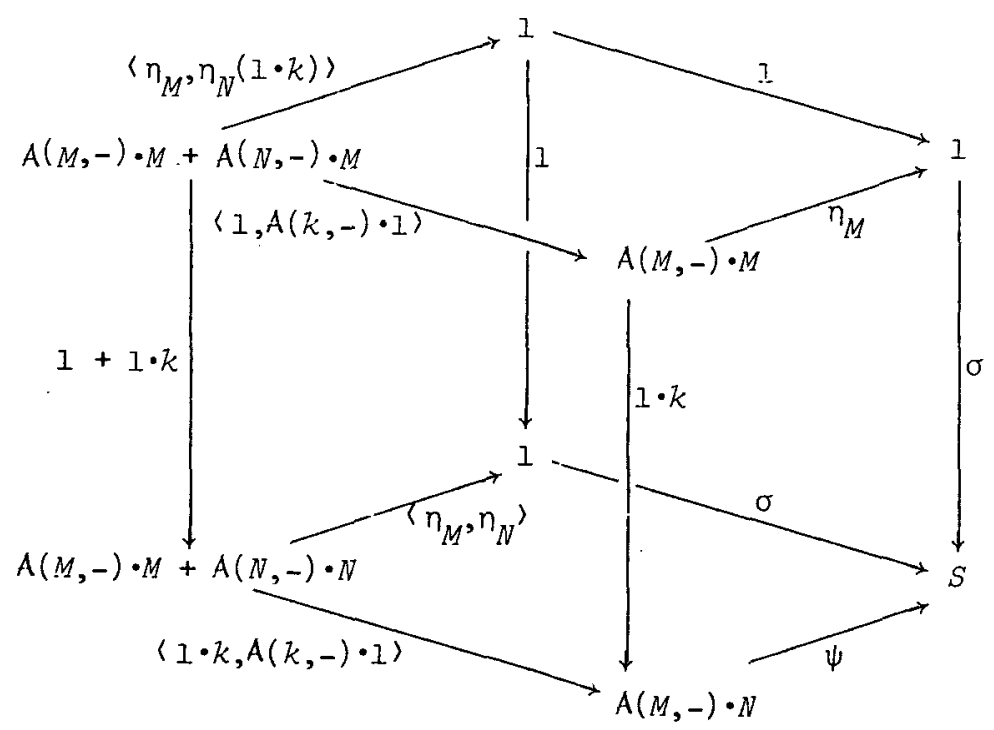

Since 1 and $1 \cdot k$ lie in $k^{+}$, so too does $\sigma$.

THEOREM 11.5. Let the hypotheses be as in Theorem 10.1, with A being $E^{\prime}$-cowelzpowered. Then $(F, N)=\left(K^{\downarrow \uparrow}, K^{\downarrow}\right)$ is a factorization system on $\mathrm{A}$, and $\mathrm{F}=K^{+}$.

Proof. Clearly $K^{+} \subset F$, since $F$ has the desired closure properties (cf. [7], Proposition 2.1.1). We argue just as before, except for the appeal to pullbacks in the proof of Proposition 11.2, when we are to show that the reflexion $p$ is in $F$; we replace this by a direct argument that $p \in K^{+}$. 
The $\sigma: 1 \rightarrow S$ of Theorem 10.1 is in $K^{+}$since it is the colimit of the $\sigma_{i}$, which are in $K^{+}$by Lemma 11.4. Hence the maps $\hat{S}_{0}^{\beta}$ of $\$ 5.2$ are in $K^{+}$by an evident induction, so that by Proposition 5.3 and Theorem 6.2, the reflexion of $A$ into $K^{\perp}$ is in $K^{+}$.

What we really need is the same conclusion when $A$ is replaced by $\mathrm{A} / E$ and $K$ by $K_{E}$; and we do get this since, colimits being formed in $\mathrm{A} / E$ as in $\mathrm{A}$, each map in $K_{E}^{+}$is clearly in $K^{+}$when seen as a map in A.

The case $M^{\prime}=$ all maps of this last result, with a slightly lesssharp description of $F$, is Theorem 4.1 of Bousfield [5].

\section{Continuous functors}

The question of "continuous functors" was reduced by Freyd and Kelly [7] to that of an orthogonal subcategory.

By a cylinder $(J, X): P \rightarrow Q$ in a small category $K$ we mean functors $P: P \rightarrow K$ and $Q: Q \rightarrow K$ with small domains, a functor $J: Q \rightarrow P$, and a natural transformation $X: P J \rightarrow Q$. A functor $F: K \rightarrow A$, where $A$ is complete, is said to be continuous with respect to $(J, X)$ if the induced map

$$
\lim F P \underset{J_{*}}{\longrightarrow} \lim F P J \underset{\lim F \chi}{\longrightarrow} \lim F Q
$$

is an isomorphism. A cylinder with $P$ the unit category 1 is just a projective cone over $Q$, and $F$ is continuous with respect to it when $F$ sends it to a limit-cone in $A$. Those functors $F$ continuous with respect to each cylinder $(J, X)$ of some family $\Gamma$ form a full replete subcategory $[K, A]_{\Gamma}$ of the category $[K, A]$; and the question arises of its reflectivity.

Let $\Delta: A \rightarrow[P, A]$ and $\delta: A \rightarrow[2, A]$ denote the diagonal functors. To say that (12.1) is an isomorphism is to say that $A(A,-)$ of it is an isomorphism for each $A \in A$; which by the definition of limit is to say that each

$$
(\Delta A, E P) \underset{[1, J]}{\longrightarrow}(\delta A, F P J) \underset{(1, F X)}{\longrightarrow}(\delta A, F Q)
$$


is an isomorphism, where the parentheses denote homs in $[P, A]$ and $[2, A]$. In this form the problem can be stated without supposing $A$ to be complete.

Of course we retain our blanket hypothesis that $A$ is cocomplete. Then $(\triangle A, F P)$ in (12.2) may be replaced by $[K, A]\left(\operatorname{Lan}_{P} \Delta A, F\right)$, and similarly for the other terms. This left Kan extension $\operatorname{Lan}_{P} \Delta A$ of $\triangle A$ along $P$ is easy to compute: it is just $P^{*} \cdot A$, where $P^{*} \in[K$, Set $]$ is the colimit of the functor $P^{\circ p} \rightarrow[K$, Set $]$ sending $p \in P$ to $K(P p,-)$. In this way we see that $[K, A]_{\Gamma}$ consists of these $F \in[K, A]$ which are orthogonal to $(J, X)^{*} \cdot A$ for each $(J, \chi) \in \Gamma$ and each $A \in A$, where $(J, X)^{*}$ is the induced map

$$
Q^{*} \underset{\chi^{*}}{\rightarrow}(P J) * \overrightarrow{J^{*}} P^{*} \text {. }
$$

Now suppose that $A$ has, in the sense of [7], $\$ 2.5$, a small generating set $G$ with respect to some proper factorization system $(E, M)$. Freyd and Kelly give in [1], Lemma 5.1.1, an easy proof that $[K, A]_{\Gamma}$ consists of those $F$ orthogonal to each $(J, X) * \cdot G$ with $G \in G$ and also orthogonal to a certain set $\Phi$ of maps in $E$; and they further show in Remark 5.1.2 that orthogonality to $\Phi$ is an automatic consequence of the rest if $G$ is also a generating set for the factorization system (extremal epimorphisms, monomorphisms).

An $A$ with a small generating set is $M$-wellpowered if it admits finite intersections of M-subobjects ([7], Corollary 2.5.2); in this case the maps (12.3) have but a small set of different $M$-parts, in the sense of the factorization (10.2), if there are but a small number of different $P: P \rightarrow K$; in particular if all but a small set of the cylinders in $\Gamma$ are cones.

We therefore have the following, which slightly generalizes in various ways Theorem 5.2.1 of [7]; the factorization systems involved are as usual supposed cocomplete:

THEOREM 12.1. Let A have the small generating set $G$ for the proper factorization system $(E, M)$. If $\Gamma$ is small and $G$ is also a generator for the system (extremal epimorphisms, monomorphisms), $[K, A] \Gamma$ is reflective under the hypothesis ( $P$ ) of $\$ 10$ (for some ( $\left.E^{\prime}, M^{\prime}\right)$ ). If 
$A$ is M-well-powered - as when it admits finite M-intersections $[K, A]_{\Gamma}$ is reflective when the number of different $P$ is small, under the hypothesis (B) of $\$ 10$ - if either $E^{\prime}=E$ or $A$ is $E$ - and $E^{\prime}$-cowe lzpowered.

\section{Functors sending given maps into $M$}

To ask of a functor $F: K \rightarrow A$ from a small $K$ that it send a given morphism $\psi$ of $K$ to a monomorphism in $A$, is to ask that it send the diagram

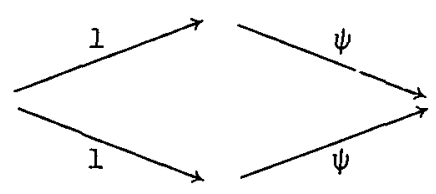

in $K$ to a pullback in $A$; which falls under the considerations of $\S 12$ above. Koubek has observed in the manuscript [11] that problems of initial automata lead to the more general consideration of those $F: K \rightarrow A$ for which $F \psi$ lies in the $M$ of some factorization system $(E, M)$ on $A$.

$\psi: 2 \rightarrow K$ induces a functor $V=[\psi, 1]:[K, A] \rightarrow[2, A]$ with a left Kan adjoint $G$. The full replete subcategory $B$ of $[2, A]$ determined by those $f: 2+A$ with $f \in M$ is reflective, the reflexion of an arbitrary $f$ being $(p, 1): f \rightarrow j$ in

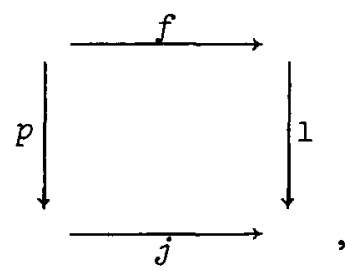

where $f=j p$ is the $(E, M)$-factorization of $f$; this is immediate from the unique-diagonal-fill-in property. Thus the corresponding idempotent monad $(R, \rho)$ has $R f=j$ and $\rho f=(p, 1)$.

It follows from Proposition 9.2 that the full subcategory $[K, A]_{\psi}$ of $[K, A]$ given by those $F: K \rightarrow A$ with $F \Psi \in M$ consists of the $S$-algebras, where $S$ is given by (9.1) with $(R, \rho)$ for $\left(S^{\prime}, \sigma^{\prime}\right)$. Since $\rho \in E$, and since $G E \subset E$ because (clearly) $V M \subset M$, we conclude 
that $\sigma \in E$.

Now consider a set $\Psi=\left(\Psi_{i}\right)$ of maps in $K$, along with a factorization system $\left(E_{i}, M_{i}\right)$ on $A$ for each $i$; and let $[K, A]_{\Psi}$ consist of those $F$ such that $F \psi_{i} \in M_{i}$ for each $i$. If we now write $\sigma_{i}: 1 \rightarrow S_{i}$ for the above $\sigma: 1 \rightarrow S$ when $\psi_{i}$ replaces $\psi$, and then define a new $\sigma: 1 \rightarrow S$ as the fibred coproduct of the $\sigma_{i}$, we have $[K, A]_{\Psi}=S-\mathrm{Alg} ;$ provided that $S$ exists.

$S$ certainly does exist if there is some cocomplete factorization system $(E, M)$ on $A$ such that $E_{i} \subset E$ for each $i$; and then $\sigma \in E$. From Remark 8.2 and Proposition 8.1 , we have the following result, which includes Theorem III.7 of Koubek [11].

THEOREM 13.1. If each $E_{i} \subset E$, where $(E, M)$ is cocomplete, then $[K, A]_{\Psi}$ is reflective in $[K, A]$.

\section{ALGEBRAS FOR GENERAL POINTED ENDOFUNCTORS}

\section{Reduction of the pointed to the well-pointed case}

14.1. For any endofunctor $T$ of $A$ we have the comma category $T / A$; an object is a triple $(A, \alpha, B)$ where $A, B \in A$ and $a: T A \rightarrow B$; a map $(A, a, B) \rightarrow\left(A^{\prime}, a^{\prime}, B^{\prime}\right)$ is a pair $\left(f: A \rightarrow A^{\prime}, g: B \rightarrow B^{\prime}\right)$ such that $g \cdot a=a^{\prime} \cdot T f$.

The category $T / A$ is cocomplete; a functor $K \rightarrow T / A$ is given by functors $X, Y: K \rightarrow A$ and a natural transformation $x: T X \rightarrow Y$, and its colimit is given by $(\operatorname{colim} X, a, B)$, where

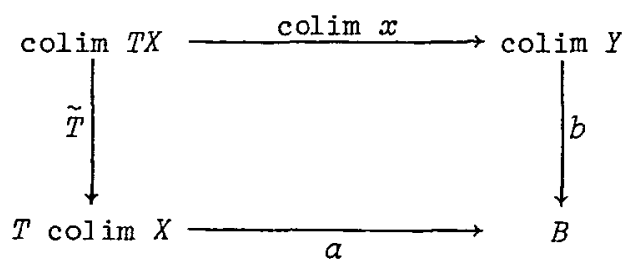

is a pushout, $\tilde{T}$ being the canonical comparison map. 
A natural transformation $\alpha: T^{\prime} \rightarrow T$ induces a functor $\alpha^{*}: T / A \rightarrow T^{\prime} / A$ sending $(A, a, B)$ to $(A, a \cdot \alpha A, B)$. This functor has a left adjoint $\alpha_{*}: T^{\prime} / A \rightarrow T / A$ which sends $(C, C, D)$ to $(C, \bar{c}, \bar{D})$ given by the pushout

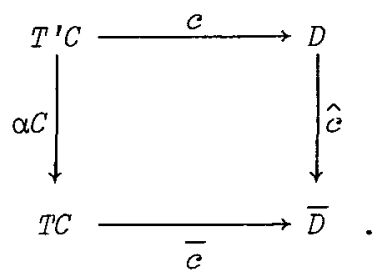

The $(C, c, D)$-component of the unit $\eta: 1 . \rightarrow \alpha^{*} \alpha_{*}$ of this adjunction is given by

$$
\eta=(1, \hat{c}):(C, c, D) \rightarrow(C, \bar{c} \cdot \alpha C, \bar{D}) ;
$$

and the $(A, \alpha, B)$-component of the counit $\varepsilon: \alpha_{*} \alpha^{*} \rightarrow 1$ is given by

$$
\varepsilon=(1, \tilde{a}):(A, \bar{c}, \bar{B}) \rightarrow(A, a, B)
$$

where, in (14.2), we take $C=A, D=B, c=a \cdot \alpha A$, and $\tilde{a}$ is determined by

$$
\tilde{a} \bar{c}=a, \quad \tilde{a} \hat{c}=1 .
$$

Our concern below is with pointed endofunctors $(T, \tau)$ on A. By a map $\alpha:\left(T^{\prime}, \tau^{\prime}\right) \rightarrow(T, \tau)$ of pointed endofunctors we mean a natural transformation $\alpha: T^{\prime} \rightarrow T$ satisfying $\alpha \tau^{\prime}=\tau$. Note that $I / A$ is just the functor category $A^{2}$; and that for a pointed $(T, \tau)$ the adjunction above has the special case

$$
\tau_{*}-1 \tau^{*}: T / A \stackrel{A^{2}}{ } .
$$

14.2. For any pointed endofunctor $(T, \tau)$ we have the category $T-A l g$ of its algebras, as defined in $\$ 5.1$. The functor $J: T-A l g \rightarrow T / A$ sending $(A, a)$ to $(A, a, A)$ and $f$ to $(f, f)$ is a full embedding; for any map $(f, g): J(A, a) \rightarrow J\left(A^{\prime}, a^{\prime}\right)$ must have $g=f$, as a consequence of $a \cdot \tau A=1$ and $a^{\prime} \cdot \tau A^{\prime}=1$. We henceforth regard $J$ as an inclusion. $T-A l g$ is evidently closed under limits in $T / A$; we are concerned with the question of its reflectivity. Clearly

PROPOSITION 14.1. A functor $K \rightarrow T-A l g$, given by a functor $X: K \rightarrow A$ and an action $x: T X \rightarrow X$, admits a colimit in T-Alg if and 
only if, when we form the pushout (14.1) with $Y=X$, the object (colim $X, a, B$ ) admits a reflexion in T-Alg. In particular, $T$-Alg is cocomplete if it is reflective in $T / A$.

If $\alpha:\left(T^{\prime}, \tau^{\prime}\right) \rightarrow(T, \tau)$ is a map of pointed endofunctors, the functor $\alpha^{*}: T / A \rightarrow T^{\prime} / A$ of $\$ 14.1$ clearly restricts to a functor $\alpha^{\# \prime}: T$-Alg $\rightarrow T^{\prime}-\mathrm{Alg}$. For a $T$-algebra $A=(A, a)$ and a $T^{\prime}$-algebra $B=(B, b)$ we have $T^{\prime}-A I g\left(B, \alpha^{\#} A\right) \cong T^{\prime} / A\left(J^{\prime} B, \alpha^{*} J A\right) \cong T / A\left(\alpha_{*} J^{\prime} B, J A\right)$. Hence:

PROPOSITION 14.2. $\alpha^{\#}: T-A l g \rightarrow T^{\prime}-A l g$ has a left adjoint $\alpha_{\#}$ if and only if, for each T'-algebra $B$, the object $\alpha_{*}^{J} B$ of T/A admits a reflexion in $\mathrm{T}-\mathrm{Alg}$. In particular the left adjoint $\alpha_{\#}$ exists if $\mathrm{T}-\mathrm{Alg}$ is reflective in $T / A$.

This has the special case where $T^{\prime}=1$ and $\alpha=\tau$. The category $1-A l g$ (for the pointed endofunctor 1 ) is just $A$, and the embedding $J^{\prime}: 1-A l g \rightarrow I / A$ is just the diagonal embedding $\Delta: A \rightarrow A^{2}$. of course $A$ is both reflective and coreflective in $A^{2}$, the domain and codomain functors $d_{0}, d_{1}: A^{2} \rightarrow A$ are respectively the right and left adjoints of $\Delta$. Clearly $\tau^{\#}: T-A l g \rightarrow l-A l g$ is nothing but the forgetful functor $U: T-A l g \rightarrow A$ of $\S 5.1$. Since $\tau_{*} \Delta B$, by $(14.2)$, is $\left(B, 1_{T B}\right.$, TB) , we have:

PROPOSITION 14.3. Free T-algebras exist - that is, the forgetful functor $U: T-A l g \rightarrow A$ has a left adjoint - if and only if, for each $B \in A$, the object $\left(B, I_{T B}, T B\right)$ of $T / A$ admits a reflexion in $T-A-g$; which is certainly so if $T-A l g$ is reflective in T/A.

The full subcategory $T-A l g$ of $T / A$ is not replete; clearly $(A, a, B)$ is isomorphic to a $T$-algebra precisely when $a \cdot \tau A$ is an isomorphism. In particular $A$, embedded by $\Delta$, is not replete in $A^{2}$, its repletion consisting of those $(A, a, B)$ with $a$ an isomorphism. These latter objects are of course the algebras for the idempotent monad $(R, \rho)$ on $A$ arising from the adjunction $d_{1} \rightarrow \Delta: A \rightarrow A^{2}$; here 
$R=\Delta d_{1}$ sends $(A, a, B)$ to $\left(B, I_{B}, B\right)$, and the component of its unit $\rho: 1 \rightarrow R$ is $(a, 1)$.

It follows that the object $(A, a, B)$ of $T / A$ is isomorphic to a $T$-algebra if and only if its image $\tau^{*}(A, a, B)$ in $A^{2}$ is an $R$-algebra. Hence, if we define a pointed endofunctor $(S, \sigma)$ on $T / A$ by the pushout

$(14.7)$

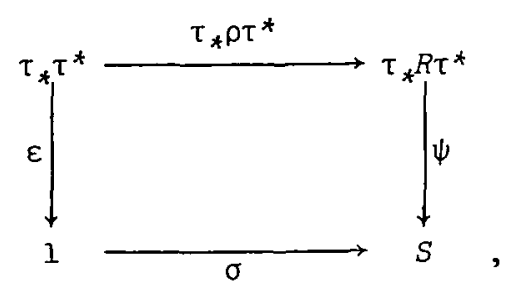

we conclude from Proposition 9.2 that:

THEOREM 14.4. $S$ is a well-pointed endofunctor on $T / A$ and $S$-Alg is the repletion of T-Alg.

Thus an object $(A, a, B)$ of $T / A$ admits a reflexion into $T-A l g$ if and only if it admits one into $S-A l g$; we say that the first reflexion exists constructively if the second does, in the sense of $\$ 5.2$; and we say that $T-A l g$ is constructively reflective in $T / A$ when this is so for every $(A, a, B)$. We further say that a colimit in T-Alg, or a left adjoint $\alpha_{\#}$, or a free T-algebra, exist constructively when the reflexions needed in Proposition 14.1-14.3 do so.

\section{The existence theorem in the pointed case}

15.1. We now show that a factorization system $(E, M)$ on $A$ induces one (with the same name) on T/A.

First, we certainly have one, defined pointwise, on the functor category $A^{2}$; the map $(f, g)$ is in $E$ or $M$ precisely when both $f$ and $g$ are.

Next, we consider the adjunction $\tau_{*} \rightarrow \tau^{*}: T / A \rightarrow A^{2}$, and the prefactorization system $(E, M)$ on $T / A$ whose $E$-part is generated by the $\tau_{*}(f, g)$ with $f, g \in E$. By [7], Lemma 4.2.1, we have

PROPOSITION 15.1. A map $(f, g)$ in $T / A$ is in $M$ if and only if, 
as maps in $A, f \in M$ and $g \in M$.

Finally, we show that this is indeed a factorization system on $T / A$, and we describe $E$ explicitly. For any $(p, q):(A, a, B) \rightarrow\left(A^{\prime}, a^{\prime}, B^{\prime}\right)$ in $T / A$ define $r$ as the connecting map in

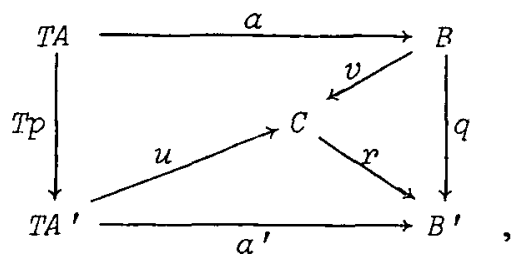

where the top quadrangle is a pushout.

PROPOSITION 15.2. ( $E, M)$ is a factorization system on $T / A$, and $(p, q) \in E$ if and only if, as maps in $A, p \in E$ and $r \in E$, where $r$ is the connecting map in (15.1).

Proof. It is easy to verify directly, using the unique-diagonal-fillin criterion, that in the situation of (15.1) with $C$ the pushout, $(p, v):(A, a, B) \rightarrow\left(A^{\prime}, u, C\right)$ is in $E$ if $p \in E$ and $(I, r):\left(A^{\prime}, u, C\right) \rightarrow\left(A^{\prime}, a^{\prime}, B^{\prime}\right)$ is in $E$ if $r \in E$. We conclude that $(p, q) \in E$ if $p \in E$ and $r \in E$.

It remains to show that every map $(f, g):(A, a, B) \rightarrow\left(A^{\prime \prime}, a^{\prime \prime}, B^{\prime \prime}\right)$ factorizes as a $(p, q)$ with $p \in E$ and $r \in E$, followed by an $(i, j)$ with $i, j \in M$. Begin by letting $i p$ be the $(E, M)$-factorization of $f$. Then define $v, u, C$ by the pushout in (15.1), and define $h: C \rightarrow B^{\prime \prime}$ by $h v=g$, $h u=a^{\prime \prime} \cdot T i$. Finally let $j r$ be the $(E, M)$-factorization of $h$, and set $a^{\prime}=r u$.

PROPOSITION 15.3. If every $E$ in $A$ is an epimorphism, or every $M$ a monomorphism, or both, the same is true of $T / A$. If $A$ is E-cowellpowered or M-wellpowered, the same is true of $T / A$. If the factorization system $(E, M)$ on $A$ is cocomplete, so is that on $T / A$.

Proof. All the verifications are immediate or straightforward, unless perhaps the last. For this, consider a family

$$
\left(p_{i}, q_{i}\right):(A, a, B) \rightarrow\left(A_{i}^{\prime}, a_{i}^{\prime}, B_{i}^{\prime}\right)
$$

of maps in $E$, and use the notation of (15.1) decorated with subscripts. 
Let the cointersection of the $p_{i}$ be given by $t_{i}: A_{i} \rightarrow A_{\infty}$. Form the pushouts

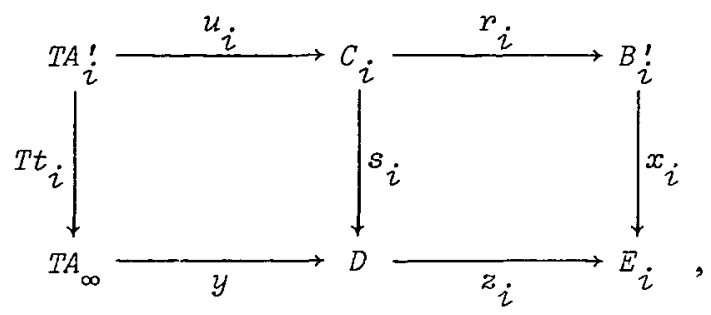

observing that $y$ and $D$ are independent of $i$ because $y$ is the pushout of $a$ by $T t_{i} \cdot T p_{i}$, which is independent of $i$. The $z_{i}$ are in $E$ as the pushcuts of the $r_{i}$; let their cointersection be given by $w_{i}: E_{i} \rightarrow B_{\infty}$. Write $a_{\infty}$ for the common value of the $w_{i} z_{i} y$; it is now easy to verify that $\left(t_{i}, w_{i} x_{i}\right):\left(A_{i}^{\prime}, a_{i}^{\prime}, B_{i}^{\prime}\right) \rightarrow\left(A_{\infty}, a_{\infty}, B_{\infty}\right)$ is the cointersection of the $\left(p_{i}, q_{i}\right)$.

PROPOSITION 15.4. For any map $\alpha: T^{\prime} \rightarrow T$ of pointed endofunctors, we have $\alpha^{*} M \subset M$ and $\alpha_{*} E \subset E$.

Proof. Since $\alpha^{*}$ is given on maps by $\alpha^{*}(f, g)=(f, g)$, the first statement is immediate; the second is then an evident consequence.

15.2. We now consider two factorization systems $(E, M)$ and $\left(E^{\prime}, M^{\prime}\right)$ on $A$, along with the induced ones on $T / A$.

PROPOSITION 15.5. If $T: A+A$ preserves, for some regular cardinal $\alpha$, the E-tightness of $\left(M^{\prime}, \alpha\right)$-cones, so does $S: T / A \rightarrow T / A$.

Proof. It suffices by $\$ 2.4$ to show that the vertices of (14.7), other than $S$, have the corresponding property. Since $\tau_{*}$ preserves colimits and (by Proposition 15.4) maps $E$ into $E$, and since the same is clearly true of $R$, it remains to prove that $\tau^{*}$ has the corresponding property.

Consider an $\alpha$-chain in $T / A$ given (as in \$14.1) by $X, Y: \alpha \rightarrow A$ and $x: T X+Y$; and an E-tight $M^{\prime}$-cone over it, with vertex $\left(A^{\prime}, a^{\prime}, B^{\prime}\right)$ and generators $t_{B}: X_{B} \rightarrow A^{\prime}, s_{B}: Y_{B} \rightarrow B^{\prime} ;$ giving $\bar{t}: \operatorname{colim} X+A^{\prime}$ and $\bar{s}: \operatorname{colim} Y \rightarrow B^{\prime}$. Since the $E$ of $A^{2}$ is given pointwise, as are colimits there, and since $\tau^{*}\left(t_{B}, s_{B}\right)=\left(t_{B}, s_{B}\right)$, what 
we have to show is that $\bar{t}, \bar{s} \in E$.

Form as in (14.1) the colimit in $T / A$, and write $A$ for $\operatorname{colim} X$. Let the comparison map in $T / A$, from the colimit to the vertex, be $(p, q)$; and let this be analyzed as in (15.1). Then the tightness in $T / A$ is expressed by $(p, q) \in E$, or by $p, r \in E$.

Since $\bar{t}$ is in fact $p$, we have $\bar{t} \in E$. Since, by Proposition 15.1, $\left(t_{\beta}\right)$ is also an $\left(M^{\prime}, \alpha\right)$-cone, the hypothesis on $T$ gives the tightness of $\left(T t_{\beta}\right)$; or equivalently that $T p \cdot \tilde{T} \in E$. Since $v$ and $b$ are the pushouts of $T p$ and $\tilde{T}$ respectively, it follows that $v b \in E$ as the pushout of $T p \cdot \tilde{T}$. Hence $r v b \in E ;$ but this is just $\bar{s}$.

Theorems 6.2 and 7.5 now give our central result:

THEOREM 15.6. Let $T$ preserve, for some regular $\alpha$, the $E$-tightness of $\left(M^{\prime}, \alpha\right)$-cones. If $A$ is $E$ - and $E^{\prime}$-cowellpowered, $T-A l g$ is constructively reflective in T/A. Even without the cowellpoweredness, it is still reflective if $M^{\prime}=M$ or if $E$ is the isomorphisms.

\section{Pointed endofunctors with $T E \subset E$}

16.1. We now apply $\S 8$ to get partial results, without the full hypotheses of Theorem 15.6, in the special case $T E \subset E$.

PROPOSITION 16.1. When $T E \subset E$, a map $(p, q)$ in $T / A$ is in $E$ if and only if $p, q \in E$. Moreover we have $S E \subset E$.

Proof. The first statement follows easily from Proposition 15.2; given that $p \in E$, the map $T p$ and hence its pushout $v$ in (15.1) are in $E$, whence $q \in E$ if and only if $r \in E$.

It follows from this that $\tau * E \subset E$. Since $E$ is in any case preserved by $\tau_{*}$ and by $R$, it is preserved by each vertex of (14.7) other than $S$; since $E$ is closed under colimits, it is preserved by $S$ too.

Propositions 8.1 and 8.3 now give:

PROPOSITION 16.2. When $T E \subset E,(A, a, B)$ admits a reflexion into $T$-Alg, which in fact lies in $E$, whenever $\sigma(A, a, B) \in E$; and if $(p, q):(A, a, B) \rightarrow\left(A^{\prime}, a^{\prime}, B^{\prime}\right)$ is in $E$, then $\left(A^{\prime}, a^{\prime}, B^{\prime}\right)$ admits $a$ 
reflexion if $(A, a, B)$ does. In the E-cowellpowered case, we can add "constructively" throughout.

Since $\sigma(A, a, B)$ is the pushout (14.7) of $\tau_{*} \rho \tau^{*}(A, a, B)$, since $\tau_{*} E \subset E$, and since $\rho \tau^{*}(A, a, B)=(a \cdot \tau A, 1)$, we have:

PROPOSITION 16.3. When $T E \subset E,(A, a, B)$ has an E-reflexion into $T$-Alg whenever $a \cdot \tau A \in E$.

of course no consequences flow from $T E \subset E$ if $E$ is the isomorphisms. To get sharper results we now suppose that $(E, M)$ is proper.

PROPOSITION 16.4. When $(E, M)$ is proper, the map $\psi: \tau_{*} R \tau^{*} \rightarrow S$ of (14.7) is in $E$ (whether $T E \subset E$ or not).

Proof. $\psi$ is the pushout of $\varepsilon$; and each component of $\varepsilon$ is in $E$, being by (14.4) and (14.5) of the form $(1, \tilde{a})$ where $\tilde{a}$ is a retraction.

The following generalizes, to the pointed-endofunctor case, Theorem 9.3 of Koubek and Reiterman [13].

THEOREM 16.5. Let $T E \subset E$ where $(E, M)$ is proper. Then $(A, a, B) \in T / A$ has a reflexion in $T-A l g$ if the free $T$-algebra on $B$ exists. Thus T-Alg is reflective in $T / A$ if and only if $U: T-A l g \rightarrow A$ has a left adjoint. When $A$ is E-cowellpowered, we can add "constructive" to the conclusions if we add it to the hypotheses.

Proof. ( $A, a, B)$ admits a reflexion (or a constructive reflexion) into $T$-Alg if $S(A, a, B)$ does, by $\S 5.2$; and $S(A, a, B)$ does if $\tau_{*} R \tau^{*}(A, a, B)$ does, by Propositions 16.4 and 16.2. But $\tau_{\star} R \tau^{*}(A, a, B)=\left(B, 1_{B}, T B\right) ;$ and to give a reflexion of this is, by Proposition 14.3 , to give the free T-algebra on $B$.

REMARK 16.6. As Koubek and Reiterman point out, this result is false without the hypothesis that $T E \subset E$ for some proper $(E, M)$; for Adámek [1] has given an example where free T-algebras exist constructively, but $T$-Alg is not cocomplete, lacking even coequalizers.

On the other hand, $T E \subset E$ ensures coequalizers in $T$-Alg, whether free T-algebras exist or not; cf. [13], Corollary 9.6. More generally: THEOREM 16.7. Let $T E \subset E$ for a proper $(E, M)$. Then a functor 
$K \rightarrow T$-Alg given by $X: K \rightarrow A, x: T X \rightarrow X$, has a colimit in T-Alg if $\tilde{T}: \operatorname{colim} T X \rightarrow T \operatorname{colim} X$ is in $E$. This is certainly the case when $K$ has a terminal object; thus in particular T-Alg admits coequalizers; constructively so, in the E-cowellpowered case.

Proof. By Proposition 14.1, we seek a reflexion of the $(A, a, B)$ given by (14.1), where $Y=X$ and $A$ denotes colim $X$. Clearly tA : $\operatorname{colim} X \rightarrow T \operatorname{colim} X$ is the composite of $\tilde{T}$ with colim $\tau X: \operatorname{colim} X \rightarrow \operatorname{colim} T X$. Since $x \cdot \tau X=1$, it follows from (14.1) that $a \cdot \tau A=b$. If $\tilde{T}$ is in $E$, so is its pushout $b$, and the reflexion exists by Proposition 16.3.

If $K$ has a terminal object $\beta$, the generators $q_{\beta}: X_{\beta} \rightarrow \operatorname{colim} X$ and $r_{\beta}: T X_{\beta} \rightarrow$ colim $T X$ of the colimit-cones are regular epimorphisms, and hence in $E$. Since $T q_{B}=\tilde{T} \cdot r_{\beta}$ and $T q_{B} \in E$, we have $\tilde{T} \in E$.

REMARK 16.8. TE $\subset E$ does not ensure cocompleteness of $T-A l g$; when $A=$ Set and $E=$ the epimorphisms, $T$-Alg may lack even an inctial object; as when $T=1+H$ and $H$ is the covariant-power-set functor. See Barr [2], Example 6.8, or Proposition 20.6 below.

16.2. Now consider a map $\alpha:\left(T^{\prime}, \tau^{\prime}\right) \rightarrow(T, \tau)$ of pointed endofunctors, and let $\left(S^{\prime}, \sigma^{\prime}\right)$ be the well-pointed enojofunctor on $T^{\prime} / A$ corresponding to the above $(S, \sigma)$ on $T / A$.

PROPOSITION 16.9. We have a pushout diagram

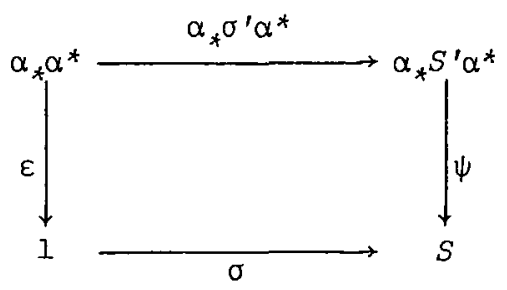

where $\varepsilon$ is the counit of the adjunction $\alpha_{*}-1 \alpha^{*}$.

Proof. $S^{\prime}$ and $\sigma^{\prime}$ are defined by the primed analogue (14.7)' of the pushout (14.7). But (14.7)' remains a pushout when we compose each vertex, on the right, with any functor; or when we compose each vertex, on the left, with a lert-adjoint functor. Thus $\alpha_{*}(14.7)^{\prime} \alpha^{*}$ is again a pushout; it exhibits $\alpha_{*} \sigma^{\prime} \alpha^{*}$ as the pushout by $\alpha_{*} \varepsilon_{\tau}, \alpha^{*}$ of $\alpha_{*} \tau_{*}^{\prime} \rho \tau^{\prime *} \alpha^{*}=\tau_{*} \rho \tau^{*}$; 
we are using subscripts here to distinguish one $\varepsilon$ from another.

If we suppose for the moment that $S$ and $\sigma$ are defined by the pushout (16.1), then pasting $\alpha_{*}(14.7)^{\prime} \alpha^{*}$ on top of (16.1) exhibits $\sigma: 1 \rightarrow S$ as the pushout of $\tau_{*} \rho \tau^{*}$ by $\varepsilon_{\alpha} \cdot \alpha_{*} \varepsilon_{\tau}, \alpha^{*}=\varepsilon_{\tau}$; so that this $S, \sigma$ is indeed that originally defined by the pushout (14.7).

It now follows from Proposition 9.2 that the $\theta: \alpha_{*} S^{\prime} \rightarrow S \alpha_{*}$, corresponding to the $\psi$ of (16.1), satisfies (8.1), where $G=\alpha_{*}$.

PROPOSITION 16.10. If $(E, M)$ is proper and $\alpha \in E$ we have $\theta \in E$.

Proof. It is immediate from the definition of maps in $T / A$ that $\alpha^{*}$, which is always faithful, is also full when $\alpha$ is epimorphic. Hence the $\varepsilon$ of (16.1) is an isomorphism; so too is its pushout $\psi$. Since $\theta$ is the composite

$$
\alpha_{*} S^{\prime} \underset{\alpha_{*} S^{\prime} \eta}{\longrightarrow} \alpha_{*} S^{\prime} \alpha^{*} \alpha_{*} \underset{\psi \alpha_{*}}{\longrightarrow} S \alpha_{*} \text {, }
$$

we have only to show that $\alpha_{*} S^{\prime} \eta \in E$; since $\alpha_{*} E \subset E$, it suffices to show $S^{\prime} \eta \in E$. By Proposition 16.4, $S^{\prime}$ is an E-quotient of $\tau_{*}^{\prime} R \tau^{\prime *}$, so it suffices in fact to show that $\tau_{*}^{\prime T \tau^{\prime *}} \eta \in E$, and therefore to show that $R \tau^{\prime *} \eta \in E$.

By (14.3), $\eta$ has the form $(1, \hat{c})$; hence $\tau^{\prime *} \eta$ has the same form, and $R \tau^{* *} \eta=(\hat{c}, \hat{c})$. This is in $E$ because $\hat{c}$, being by (14.2) the pushout of $\alpha C$, is in $E$.

Proposition 8.4, along with Propositions 14.2 and 14.3, now gives the following, which generalizes to the pointed case Corollary 9.5 of Koubek and Reiterman [13] and the results on pages 65 and 66 of Reiterman [15]:

THEOREM 16.11. Let $T E \subset E$ for a proper $(E, M)$ and let $\alpha:\left(T^{\prime}, \tau^{\prime}\right) \rightarrow(T, \tau)$ be in $E$. Then if an object $(A, a, B)$ of $T^{\prime} / A$ has a reflexion into $T^{\prime}-\mathrm{Alg}$, the object $\alpha_{*}(A, a, B)$ of $T / A$ has a reflexion into $T-A l g$. In particular the free $T$-algebra on $A$ exists if the free $T^{\prime}$-algebra does. Moreover the full inclusion $\alpha^{\#}: T$-Alg $\rightarrow$ T'Alg has a left adjoint $\alpha_{\#}$. If A is E-cowellpowered, we can add "constructively" throughout. 


\section{The explicit form of $S, \sigma$ for a pointed $T$}

17.1. The results above were obtained directly from the definition $(14.7)$ of $(S, \sigma)$, without an explicit calculation of it. Yet such a calculation is of interest as giving explicitly the sequence in T/A which, in the constructive case, converges to the reflexion in T-Alg.

Given $(A, a, B)$ in $T / A$, consider the coequalizer $b: T B \rightarrow C$ of $T a \cdot \tau T a$ and $T a \cdot T \tau A$ in

$$
T A \underset{T \tau A}{\stackrel{\tau T A}{\longrightarrow}} T^{2} A \underset{T a}{\longrightarrow} T B \underset{b}{\longrightarrow} C
$$

PROPOSITION 17.1. S defined by the pushout (14.7) has $S(A, a, B)=(B, b, C)$ with $b, C$ as in (17.1); the (A, $a, B)$-component of $\sigma$ is $(a \cdot \tau A, b \cdot \tau B)$; and the $(A, a, B)$-component of the transform $\phi: R \tau^{*} \rightarrow \tau^{*} S$ of $\psi$ is $(1, b \cdot \tau B)$.

Proof. Consider what it is to give maps

$$
(f, g):(A, a, B) \rightarrow\left(A^{\prime}, a^{\prime}, B^{\prime}\right)
$$

and

$$
(h, k): \tau_{*} R \tau^{*}(A, a, B) \rightarrow\left(A^{\prime}, a^{\prime}, B^{\prime}\right)
$$

in $T / A$ satisfying $(f, g) \cdot \varepsilon(A, a, B)=(h, k) \cdot \tau_{*} \rho \tau^{*}(A, a, B)$.

To give $(h, k)$ is equally to give its transform

$$
(u, v): R \tau^{*}(A, a, B) \rightarrow \tau^{*}\left(A^{\prime}, a^{\prime}, B^{\prime}\right)
$$

in $A^{2}$, under the adjunction $\tau_{*}-1 \tau^{*}$; that is,

$$
(u, v):\left(B, I_{B}, B\right) \rightarrow\left(A^{\prime}, a^{\prime} \cdot \tau A^{\prime}, B^{\prime}\right) .
$$

Then the commutativity condition above transforms to

$$
(f, g)=(u, v) \cdot \rho \tau^{*}(A, a, B)=(u, v) \cdot(a: \tau A, 1)
$$

or $f=u \cdot a \cdot \tau A$ and $g=v$.

But the condition that $(u, v)$ be a map in $A^{2}$ is $v=a^{\prime} \cdot \tau A^{\prime} \cdot u$; so that all is determined by $u$ alone. There remains the condition for $(f, g)$ to be a map in $T / A$; namely $a^{\prime} \cdot T f=g \cdot a$, or $a^{\prime} \cdot T u \cdot T a \cdot T \tau A=a^{\prime} \cdot \tau A^{\prime} \cdot u \cdot a$. Since the right side of the latter is also $a^{\prime} \cdot T u \cdot T a \cdot \tau T A$ by naturality, the condition is ultimately, by (17.1), that 
$a^{\prime} \cdot T u=w \cdot b$ for some (necessarily unique) $w: C \rightarrow B^{\prime}$.

Since this last is the assertion that $(u, w)$ is a map $(B, b, C) \rightarrow\left(A^{\prime}, a^{\prime}, B^{\prime}\right)$ in $T / A$, and since $(u, w)(a \cdot \tau A, b \cdot \tau B)=(f, g)$ and $(u, w)(l, b \cdot \tau B)=(u, v)$, the result follows.

REMARK 17.2. Since $S(A, a, B)=(B, b, C)$ where $b$ is a regular epimorphism, the value of $S$ at the map $(f, g):(A, a, B) \rightarrow\left(A^{\prime}, a^{\prime}, B^{\prime}\right)$ has the form $(g, h):(B, b, c) \rightarrow\left(B^{\prime}, b^{\prime}, C^{\prime}\right)$, where $h$ is determined by $h b=b^{\prime} \cdot T g$. Note that when $T$ is itself well-pointed, so that $\tau \tau=\tau T$, we have $S(A, a, B)=\left(B, 1_{B}, T B\right)$ and $\sigma(A, a, B)=(a \cdot \tau A, \tau B)$.

17.2. Now for any $(A, a, B)$ in $T / A$, the free-algebra sequence $\hat{S}(A, a, B)$. of $\$ 5.2$ may be called the algebra-reflexion sequence for $(A, a, B)$. We give it explicitly.

We define inductively a sequence $X: \infty \rightarrow A$ and a natural transformation $x: T X \rightarrow X^{+}$, such that the composite $x \cdot \tau X$ is the canonical map $X \rightarrow X^{+}$of $\$ 4$. This fixes the values of the non-limit connecting maps by

$$
X_{B}^{B+1}=x_{B} \cdot \tau X_{B}
$$

We start by setting

$$
X_{0}=A, \quad X_{1}=B, \quad x_{0}=a: T A \rightarrow B ;
$$

we define $x_{B+1}$ and $X_{B+2}$ to be the coequalizer in

$$
T X_{B} \underset{T \tau X_{B}}{\stackrel{\tau T X_{B}}{\longrightarrow}} T^{2} X_{B} \underset{x_{B}}{\stackrel{\longrightarrow}{T}} T X_{B+1} \underset{x_{B+1}}{\longrightarrow} X_{B+2}
$$

for a limit-ordinal $\alpha$ we set

(17.5) $X_{\alpha}=\underset{\beta<\alpha}{\operatorname{colim}} X_{\beta}$, with $X_{\beta}^{\alpha}$ the generators of the colimit-cone, and we define $x_{\alpha}$ and $X_{\alpha+1}$ to be the coequalizer in 


\section{$(17.6)$}

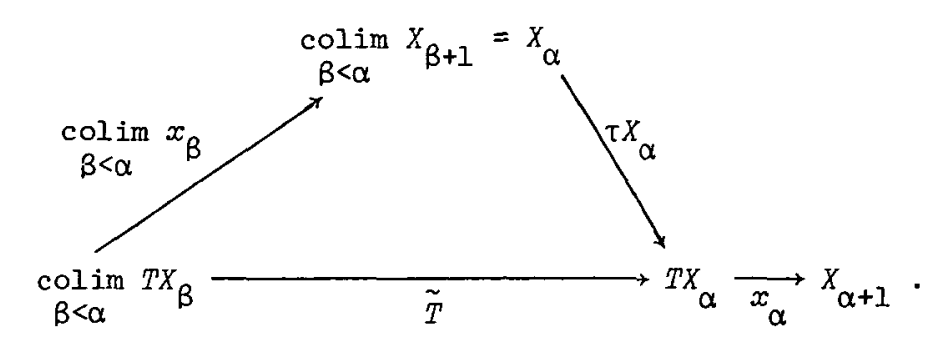

That $x$ is indeed natural follows automatically from our inductive proof of:

THEOREM 17.3. With $X, x$ as above, the sequence $\hat{S}(A, a, B): \infty \rightarrow T / A$ is $\left(X, x, X^{+}\right)$, so that

$$
S^{\beta}(A, \alpha, B)=\left(X_{B}, x_{B}, X_{B+1}\right) \text {. }
$$

The sequence $\hat{S}(A, a, B)$ converges at a precisely when the sequence $X$ does; and then (if we take for simplicity $x_{\alpha}^{\alpha+1}$ to be 1 ) the reflexion of $(A, a, B)$ in $T-A l g$ is $X_{\alpha}$ with the action $x_{\alpha}: T X_{\alpha} \rightarrow X_{\alpha+1}=X_{\alpha}$.

Proof. In view of Proposition 17.1, the only point which is not immediate is the identification of the two sequences at a limit-ordinal $\alpha$. Given the description (14.1) of colimits in $T / A$, what we are to prove is that

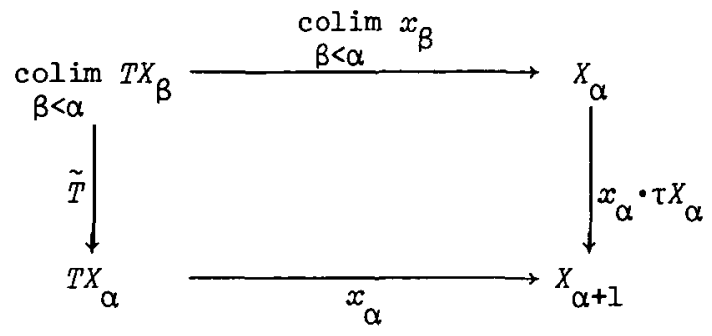

is a pushout. If $u: T X_{\alpha} \rightarrow D$ and $v: X_{\alpha} \rightarrow D$ satisfy $u \cdot \tilde{T}=\underset{\beta<\alpha}{\operatorname{colim}} x_{\beta}$, they satisfy $u \cdot T X_{\beta}^{\alpha}=v \cdot X_{\beta+1}^{\alpha} \cdot x_{\beta}$ for all $\beta<\alpha$. On composing this with $\tau X_{\beta}$, the left side becomes $u \cdot \tau X_{\alpha} \cdot X_{\beta}^{\alpha}$ by naturality, while by (17.2) the right side becomes $v \cdot X_{\beta+1}^{\alpha} \cdot X_{\beta}^{\beta+1},=v \cdot X_{\beta}^{\alpha}$. It follows that $v=u \cdot \tau X_{\alpha}$; so that $u \cdot \tilde{T}=u \cdot \tau X_{\alpha} \cdot \underset{\beta<\alpha}{\operatorname{colim}} x_{\beta}$, and the result follows from (17.6). 
REMARK 17.4. In the special case when $T$ is well-pointed, so that $T \tau=\tau T$, it is clear by induction that $X_{B+1}=T X_{B}$ and $x_{\beta}=1$, for $\beta \geq 1$; here we use Lemma 5.5 to check that, for a limit-ordinal $\alpha$, the $\tilde{T}$ in (17.6) is just $\tau X_{\alpha}$. So in this case the sequence $X^{+}$is just the free-algebra sequence $\hat{T} B$ of $\S 5.2$; and $(A, a, B)$ has a reflexion into $T$-Alg exactly when the free $T$-algebra on $B$ exists.

Thus Theorems 6.2 and 7.5 are in fact special cases of Theorem 15.6, while the results of $\S 8$ are special cases of those of $\S 16$.

\section{ALGEBRAS FOR UNPOINTED ENDOFUNCTORS}

\section{Reduction of the unpointed to the pointed case}

From any endofunctor $H$ of $A$ we get a pointed endofunctor $(T, \tau)$, by taking $T$ to be the coproduct $1+H$ and $\tau: 1 \rightarrow 1+H$ to be the injection. To give an action of $(T, \tau)$ on $A$ is just to give any map $a: H A \rightarrow A$; we call $a$ an action of $H$ on $A$, and call $(A, a)$ an H-algebra. A map of T-algebras is just an $f: A \rightarrow A^{\prime}$ with $f \cdot a=a^{\prime} \cdot H f$; we call it a map of H-algebras. Thus $T-A l g$ and $H-A l g$ are the same category; confusion between the pointed and the unpointed senses of the words is unlikely.

An object of $T / A=(1+H) / A$ is now a co-span

$$
H A \underset{m}{\longrightarrow} B \frac{\leftarrow}{n} A \text {, }
$$

the maps being pairs ( $\left.f: A \rightarrow A^{\prime}, g: B \rightarrow B^{\prime}\right)$ for which the evident diagrams commute.

Clearly $T$ preserves the E-tightness of $\left(M^{\prime}, \alpha\right)$-cones if $H$ does. Further $T E \subset E$ if $H E \subset E$. Moreover, in the case of a functor $X: K \rightarrow A$ and the comparison-map $\tilde{H}: \operatorname{colim} H X \rightarrow H \operatorname{colim} X$, we have $\tilde{T} \in E$ if $\tilde{H} \in E$. Finally, a natural transformation $\alpha: H^{\prime} \rightarrow H$ gives a map $1+\alpha: 1+H^{\prime} \rightarrow 1+H$ of pointed endofunctors, inducing an $\alpha^{\#}: H-\mathrm{Alg} \rightarrow H^{\prime}-\mathrm{Alg}$; and $(1+\alpha) \in E$ if $\alpha \in E$. Thus :

THEOREM 18.1. In Theorems 15.6, 16.5, 16.7, and 16.11, if we replace $T$ by the unpointed $H$, replace $T-A l g$ by $H-A l g$, replace $T / A$ by - 
$(1+H) / A$, and replace the hypotheses on $T, \tilde{T}$, and $\alpha$ by the same hypotheses on $H, \tilde{H}$, and $\alpha$, the conclusions still hold.

It is this case of Theorem 15.6 which generalizes Theorem 8.5 of Koubek and Reiterman [13]; while the other three theorems generalize, as we have already remarked, various results of their $\$ 9$.

The results in the pointed case are the stronger ones; for they include as above those in the unpointed case, while the converse is false.

\section{Partial algebras}

Suppose for this section that $A$ admits finite limits and is E-cowellpowered for a proper factorization system $(E, M)$.

$$
\text { A span }
$$

$$
H A \underset{u}{\leftarrow} C \underset{v}{\longrightarrow} A
$$

is called by Koubek and Reiterman [13] in their $\$ 5$ a generalized partial algebra for $H$; if a map between two such is a pair ( $f: A \rightarrow A^{\prime}, h: C \rightarrow C^{\prime}$ ) for which the evident diagrams commute, they form a category GPA. A full subcategory $N D A$ is formed by the nondeterministic algebras: those spans for which $\langle u, v\rangle: C \rightarrow H A \times A$ is in $M$. Still smaller is the full subcategory $P A$ of partial algebras, given by those spans with $u \in M$. Another full subcategory of GPA smaller than NDA is the category $R E G$ of regular generalized partial algebras, namely those for which the span (18.2) is the pullback of some cospan (18.1). Inside all of these sits the full subcategory $H-A l g$ of honest algebras, indentified with those generalized partial algebras for which $C=H A$ and $u=1$. Thus we have full inclusions

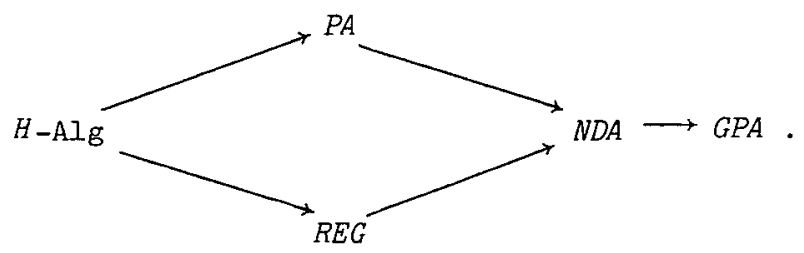

Koubek and Reiterman point out in their $\$ 5$ that there are left adjoints to the inclusion $N D A \rightarrow G P A$ (take the $(E, M)$-factorization of $\langle u, v\rangle$ ), to the inclusion $R E G \rightarrow G P A$ (take the pushout (18.1) of (18.2) and then take its pullback), and to the inclusion $P A \rightarrow G P A$ (a simple 
transfinite argument using the cowellpoweredness). It follows (this is their Proposition 6.6) that:

PROPOSITION 19.1. If any one of the fulz inclusions

$$
H-\mathrm{Alg} \rightarrow R E G, \quad H-\mathrm{Alg} \rightarrow \mathrm{PA}, \quad H-\mathrm{Alg} \rightarrow \mathrm{NDA}, \quad H-\mathrm{Alg} \rightarrow G P A
$$

has a left adjoint, so do all the others.

Now if $T$ again denotes $1+H$, the functor $K: T / A \rightarrow G P A$ sending (18.1) to its pullback (18.2) has the left adjoint $L: G P A \rightarrow T / A$ which sends (18.2) to its pushout. Since the composite of $K$ with the inclusion $J: H-A l g \rightarrow T / A$ of $\$ 14.2$ is clearly the embedding $H-A l g \rightarrow G P A$, we have:

THEOREM 19.2. The generalized partial algebra $(A, u, v, C)$ of (18.2) has a reflexion in $H$-Alg if its image $L(A, u, v, C)$ in $T / A$ does, and in particular when the algebra-reflexion sequence for this latter converges. When $H-\mathrm{Alg}$ is reflective in T/A, each of the inclusions in Proposition 19.1 has a left adjoint.

The first sentence here includes Theorem 7.I of Koubek and Reiterman, who use GPA as a well-behaved category in which to embed $H$-Alg, as we use $T / A$, and whose "completion construction" is precisely our algebrareflexion sequence applied to $L(A, u, v, C)$; as follows easily from $\$ 20$ below. We further recapture their Theorem 9.4 in the form:

PROPOSITION 19.3. If $H E \subset E$, the generalized partial algebra $(A, u, v, C)$ has a constructive reflexion in $H-A l g$ whenever $u \in E$.

Proof. If $L(A, u, v, C)$ is the pushout (18.1) of (18.2), we have $n \in E$. If $a$ denotes $(n, m): T A=A+H A \rightarrow A$, then $a \cdot \tau A=n \in E$; and the result follows from Theorem 19.2 and Proposition 16.3.

20. The explicit form of the sequence for a mere endofunctor 20.1. Given a co-span $(A, m, n, E)$ as in (18.1), consider the pushout

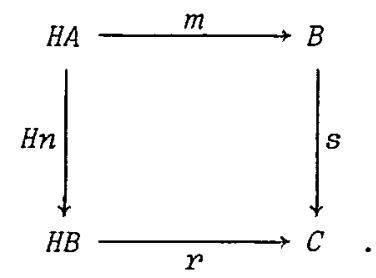


PROPOSITION 20.1. The well-pointed endofunctor $S$ of (14.7), with $T=1+H$, is given by $S(A, m, n, B)=(B, r, s, C)$; and the $(A, m, n, B)$-component of $\sigma$ is $(n, s)$.

Proof. One verifies immediately that $b=\langle s, r\rangle$ is the coequalizer in (17.1) when $a=(n, m)$.

We now consider the algebra-reflexion sequence $X$ of $\$ 17.2$ in the present case. Given $(A, m, n, B)$ as in (18.1), we define a sequence $X: \infty \rightarrow A$ and a natural transformation $y: H X \rightarrow X^{+}$. Starting with (20.2) $X_{0}=A, X_{I}=B, X_{0}^{\perp}=n: A \rightarrow B, y_{0}=m: H A \rightarrow B$, we define $x_{\beta+2}, x_{\beta+1}^{\beta+2}$, and $y_{\beta+1}$ by the pushout

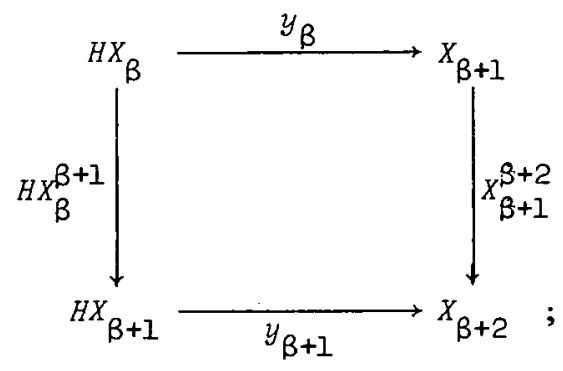

for a limit-ordinal $\alpha$ we set

(20.4) $X_{\alpha}=\underset{\beta<\alpha}{\operatorname{colim}} X_{\beta}$, with $X_{\beta}^{\alpha}$ the generators of the colimit-cone; and we define $x_{\alpha+1}, x_{\alpha}^{\alpha+1}$, and $y_{\alpha}$ by the pushout

$(20.5)$

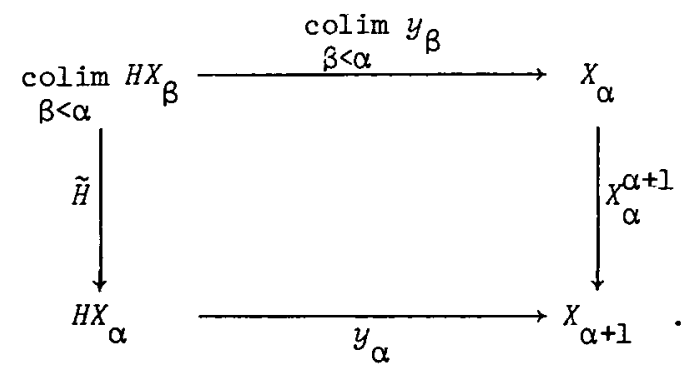

THEOREM 20.2. Let $X, y$ be as above and let $\phi: X \rightarrow X^{+}$be the canonical map with $\phi_{B}=x_{B}^{\beta+1}$. Then the sequence 
$\hat{S}(A, m, n, B)=\infty \rightarrow(1+H) / A$ is $\left(X, y, \phi, X^{+}\right)$. This sequence converges at a precisely when the sequence $X$ does; whereupon $X_{\alpha}$, with the action $y_{\alpha}: H X_{\alpha} \rightarrow X_{\alpha+1}=X_{\alpha}$, is the reflexion of $(A, m, n, B)$ into $H-\mathrm{Alg}$.

Proof. This is what Theorem 17.3 reduces to in the present case: for (17.4) reduces to $(20.3)$ by Proposition 20.1 , and it is equally immediate that $(17.6)$ reduces to $(20.5)$.

In the present case we can simplify and unify (20.3) and (20.5); for an easy inductive argument gives:

PROPOSITION 20.3. For $X, y$ as above the diagram

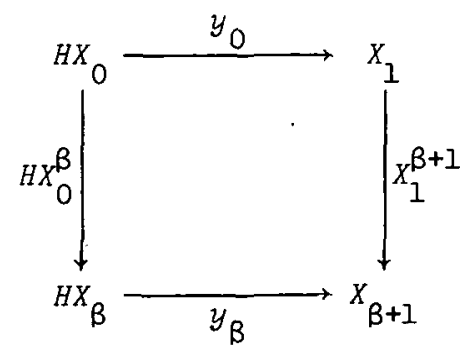

is a pushout for each $B$.

Combining this with Propositions 14.1, 14.2, and 14.3, we get simplified descriptions of what we might call the colimit sequence, the algebraic-adjoint sequence, and the free-algebra sequence, for an unpointed $H$; cf. Koubek and Reiterman [13], §3, and Reiterman [15], §1.

We give the free-algebra sequence explicitly. By Proposition 14.3 we are to apply Theorem 20.2 to the cospan

$$
H A \underset{m}{\longrightarrow} A+H A \stackrel{n}{\leftarrow} A
$$

where $m$ and $n$ are the injections. If 0 denotes the initial object, we have a pushout

$(20.7)$

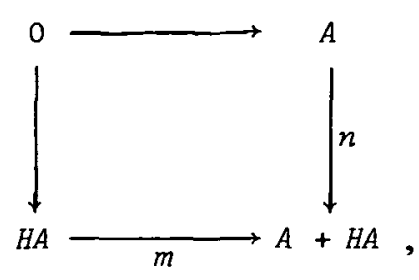


and we get another by pasting this on top of the pushout (20.6). Hence we have:

THEOREM 20.4. Given $A \in A$ define $X: \infty \rightarrow A$ and $y: H X \rightarrow X^{+}$by setting $X_{0}=A$; setting $X_{B+1}=A+H X_{B}$ with injections $X_{0}^{\beta+1}$ and $y_{\beta}$; and, for a limit-ordinal $\alpha$, setting $x_{\alpha}=\underset{\beta<\alpha}{\operatorname{colim}} x_{\beta}$ with generators $x_{\beta}^{\alpha}$. Then if $X$ converges at $\alpha$ the free H-algebra on $A$ is $X_{\alpha}$ with the action $y_{\alpha}: H X_{\alpha} \rightarrow X_{\alpha+1}=X_{\alpha}$.

20.2. Recall that an $H$-algebra $(C, C)$ embeds in $(1+H) / A$ as the cospan $(C, C, 1, C)$. A map in $(1+H) / A$ from $(A, m, n, B)$ to this is a pair $(f, g)$ of maps satisfying

(20.8)

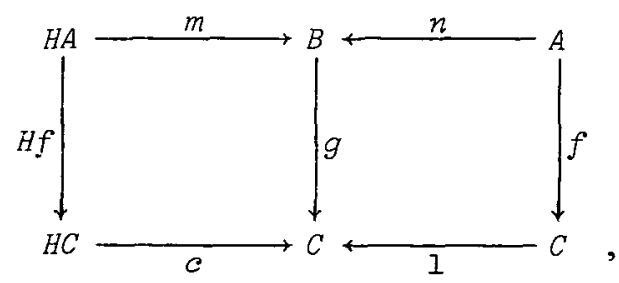

and hence equivalently a single map $g$ satisfying

$(20.9)$

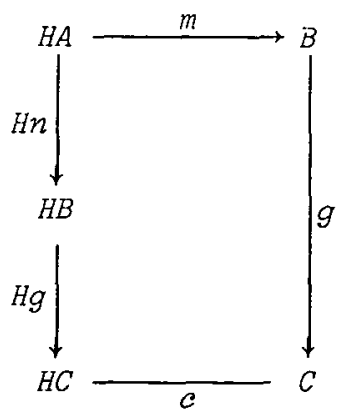

The following is an adaptation to $(1+H) / A$ of Proposition 6.7 of Koubek and Reiterman [13]:

PROPOSITION 20.5. If (20.8) is in fact the reflexion of $(A, m, n, B)$ into $H$-Alg, then (20.9) is a pushout.

Proof. Let the pushout of the top and left edges of (20.9) be given by $u: H C \rightarrow D$ and $v: B \rightarrow D$, and let $w: D \rightarrow C$ be the unique map satisfying $w u=c$ and $w v=g$. Make $D$ into an $H$-algebra by giving it 
the action $u \cdot H w: H D \rightarrow D$, and observe that $w: D \rightarrow C$ is then an algebra-map.

Since $v m=u \cdot H g \cdot H n=u \cdot H w \cdot H v \cdot H n$, it follows by analogy with (20.9) that $v$ provides a map in $(1+H) / A$ from $(A, m, n, B)$ to the $H$-algebra $D$. Since (20.8) is the reflexion, we have $v=t g$ for a unique algebramap $t: C \rightarrow D$. But now $g=w v=w t g$; whence the algebra-map $w t=1$, because $g$ gives the reflexion.

As an algebra-map, $t$ satisfies $t c=u \cdot H w \cdot H t$; since $w t=1$, this gives $u=t c=t w u$. On the other hand $v=t g=t w v$. Since $u$ and $v$ constitute the pushout, we conclude that $t w=1$. Hence $w$ is an isomorphism, and (20.9) is the pushout.

The following special case, obtained by pasting (20.7) on top of (20.9) when $B=A+H A$, is Proposition 5.11 of Barr [2]:

PROPOSITION 20.6. If the free H-algebra on $A \in A$ is (C, c), with unit $f: A \rightarrow C$, then $f$ and $c: H C \rightarrow C$ express $C$ as the coproduct $A+H C$.

REMARK 20.7. Propositions 20.5 and 20.6 seem to have no simple analogue in the pointed-endofunctor case. Of course they follow alternatively from Proposition 20.3 and Theorem 20.4 when the reflexion exists constructively; but the real point is their more general validity. In certain rather restricted cases, this leads to a necessary condition for the existence, and even for the constructive existence, of the reflexion. An example is given by Theorem 6.8 of Koubek and Reiterman [13], which is easily modified to deal with out $(1+H) / A$ instead of their GPA. Because of the very restricted applicability of this result we pass it by, and consider necessary conditions only for the important special case $A=$ Set .

\section{The special case $A=$ Set}

21.1. If $E$ is the epimorphisms, every endofunctor $H$ of Set has $H E \subset \bar{C}$, since epimorphisms are retractions. We need not have $H M C: M$; but a monomorphism $i$ with non-empty domain is a coretraction, so that $H i$ is a monomorphism.

For a cardinal $\alpha$, let us loosely write $H \alpha$ for card $H \alpha$. The 
essence of the following result is due to Kürkovâ-Pohlová and roubek [14]:

THEOREM 21.1. For an endofunctor $H$ of Set, the following are equivalent:

(i) free H-algebras exist constructively;

(ii) $H$-Alg is constructively reflective in $(1+H) /$ Set;

(iii) free H-algebras exist;

(iv) there are arbitrarily large cardinals $\gamma$ with $H \gamma \leq \gamma$.

Proof. (i) implies ( $i i)$ by Theorem 16.5, and ( $i i$ ) implies (iii) trivially; (iii) implies (iv) since, if the free $H$-algebra on the cardinal $\alpha$ has cardinal $\gamma$, Proposition 20.6 gives $\alpha+H \gamma=\gamma$, so that $\alpha \leq \gamma$ and $H \gamma \leq \gamma$. It remains to prove that (iv) implies (i).

Given a non-zero cardinal $\alpha$, we can find by (iv) an infinite cardinal $\beta$ satisfying $\alpha \leq \beta$ and $H \beta \leq \beta$, and hence satisfying $\alpha+H \beta \leq \beta$. Let $\beta$ be the least cardinal satisfying this last inequality. If $\gamma=\alpha+H \beta$ we have $0<\alpha \leq \gamma \leq \beta$; hence $H \gamma \leq H \beta$ since there is a coretraction $\gamma \rightarrow \beta$. Thus $\alpha+H \gamma \leq \alpha+H \beta=\gamma$; and since $\beta$ was the least solution we have $\gamma=\beta$, or $\alpha+H \beta=\beta$.

We first consider the free-algebra sequence $X$ of Theorem 20.4 in the case of a non-empty $A$. By the last paragraph we can find a set $B$ and an isomorphism $k: A+H B \rightarrow B$. We define inductively maps $r_{\beta}: X_{B} \rightarrow B$ forming (as is easy to verify inductively) a cone over $X$ with vertex $B$. Let $r_{0}: X_{0}=A \rightarrow B$ be the composite of $k$ with the injection $A \rightarrow A+H B$, let $r_{\beta+1}$ be the composite

$$
X_{\beta+1}=A+H X_{\beta} \underset{1+H r_{\beta}}{\longrightarrow} A+H B \underset{k}{\longrightarrow} B \text {, }
$$

and, for a limit-ordinal $\alpha$, let $r_{\alpha}: X_{\alpha}=\underset{\beta<\alpha}{\operatorname{colim} X_{\beta}} \rightarrow B$ be the induced map. Since the domain of $r_{\beta}$ is not empty, it follows inductively (using $\$ 2.2$ at the limit-ordinals) that each $r_{\beta}$ is a monomorphism. Hence the sequence $X$ converges since Set is wellpowered.

When $A$ is the empty set 0 we have $x_{0}=0$ and $X_{1}=H 0$, so that the sequence converges trivially if $H O=0$. In the contrary case the 
free $H$-algebra on $H 0$ exists constructively by the above, so that by Theorem 16.5 the reflexion in $H-A l g$ of the cospan $(H O \rightarrow O+H O+0)$ exists constructively; but by Proposition 14.3 the reflexion-sequence for this cospan is just the free-algebra sequence for $A=0$.

REMARK 21.2. The authors of [14] call $H$ non-excessive if it satisfies the necessary and sufficient condition ( $i v)$ of the above theorem. This condition is of course implied by the sufficient conditions given by Theorems 18.1 and 15.6; which, since the only cocomplete factorization systems on Set have for $E$ the isomorphisms or the epimorphisms, fall together by Proposition 2.2 into the requirement that $H$ preserve, for some regular cardinal $\alpha$, the colimits of $\alpha$-chains. This latter requirement, by Proposition 2.3 and the remark following it, is implied by the smallness of $H$; and may or may not conversely imply this, depending on the existence of very large cardinals. However non-excessivity is a strictly weaker condition - the following example, suggested by G. Monro, improves that of Koubek and Reiterman [12], $\$ 3$, in being independent of the Generalized Continuum Hypothesis.

EXAMPLE 21.3. There is a non-excessive $H$ : Set $\rightarrow$ Set which does not, for any regular cardinal $\alpha$, preserve the colimits of $\alpha$-chains.

Proof. Define inductively the sequence $\lambda$ of "beth-cardinals" by $\lambda_{0}=\omega, \lambda_{\beta+1}=2^{\lambda_{\beta}}, \lambda_{\alpha}=\sup _{\beta<\alpha} \lambda_{\beta}$ for a limit ordinal $\alpha$. Define a functor $H:$ Set $\rightarrow$ Set on objects by

$$
H A=\left\{\sum_{\gamma \in \operatorname{Lim}} \operatorname{Inj}\left(\lambda_{\gamma}, A\right)\right\}+1,
$$

where $\operatorname{Lim}$ is the set of all small limit-ordinals, where $\operatorname{Inj}(N, A)$ is the set of all injective maps $N \rightarrow A$, and where 1 is the one-element set \{0\}. For a map $f: A \rightarrow B$ let $H f$ send the injection $g: \lambda_{\gamma} \rightarrow A$ to

$f g$ if $f g$ is an injection, and to 0 otherwise; and let it send 0 to 0 .

It is immediate that $H$ is non-excessive, since for any $\beta$ we have the inequality of cardinals $H \lambda_{B+1} \leq \lambda_{B+1}$. Yet, for any regular cardinal $\alpha, H$ does not preserve the colimit of the $\alpha$-chain $X$, where $X_{B}=\lambda_{B+1}$ 
and $X_{\beta}^{\gamma}$ is the inclusion of $\lambda_{\beta+1}$ as an initial segment of $\lambda_{\gamma+1}$; for colim $H X$ has cardinal $\lambda_{\alpha}$, while $H \operatorname{colim} X=H \lambda_{\alpha}$ has cardinal $2^{\lambda} \cdot \square$

REMARK 21.4. Koubek and Reiterman give in [13], \$11, necessary and sufficient conditions, in the case $A=$ Set, for the weaker requirement that $\alpha^{\#}: H-\mathrm{Alg} \rightarrow H^{\prime}-\mathrm{Alg}$, induced by $\alpha: H^{\prime} \rightarrow H$, have a left adjoint.

21.2. Following Reiterman [15], \$4 and Koubek and Reiterman [12], §3, we can extend from $A=$ Set to certain "concrete" categories $A$, for endofunctors with $H E \subset E$, the sufficiency (but not the necessity - see [12], §3) of a cardinal-condition of the non-excessivity type; which here too may in suitable cases be weaker than conditions of the Theorem 15.6 type:

THEOREM 21.5. Let $A$ be E-cowelzpowered for a proper factorization sysiem $(E, M)$, and let $H: A \rightarrow A$ satisfy $H E \subset E$. Suppose there is an adjunction $G \rightarrow V: A \rightarrow$ Set with its counit $\varepsilon: G V \rightarrow 1$ in $E$. Then if $V H G:$ Set $\rightarrow$ Set is non-excessive, $H-A l g$ is constructively reflective in $(1+H) / A$.

Proof. By Theorem 16.5 we have only to prove that free $H$-algebras exist constructively. Let $X$ be the free- $H$-algebra sequence on $A \in A$, and $Y$ the free-VHG-algebra sequence on $V A \in$ Set. We produce a natural transformation $f: G Y \rightarrow X$ in $E$; then, since $Y$ converges by Theorem 21.1 , the convergence of $X$ follows from the $E$-cowellpoweredness.

We define $f_{\beta}$ inductively, checking inductively that it lies in $E$. For $f_{0}: G Y_{0} \rightarrow X_{0}$ we take $\varepsilon A: G V A \rightarrow A$, which lies in $E$. Using Theorem 20.4 we take for $f_{\beta+1}: G Y_{\beta+1} \rightarrow X_{\beta+1}$ the composite

$$
G\left(V A+V H G Y_{\beta}\right) \cong G V A+G V H G Y_{\beta} \underset{\varepsilon A+\varepsilon H G Y_{\beta}}{\longrightarrow} A+H G Y_{\beta} \underset{1+H f_{B}}{\longrightarrow} A+H X_{B},
$$

which is in $E$ since $\varepsilon$ and $f_{\beta}$ are. Finally for a limit-ordinal $\alpha$ we take $f_{\alpha}=\underset{\beta<\alpha}{\operatorname{colim}} f_{\beta}$, which makes sense because $G$ preserves colimits; again it lies in $E$. The naturality of $f$ is easily checked by induction.

We can moreover deduce similar results in the pointed-endofunctor 
case: we content ourselves with the following, for the case $A=$ Set :

THEOREM 21.6. For a pointed endofunctor $(T, \tau)$ of Set, T-Alg is constructively reflective in $T /$ Set if there are arbitrarily large cardinals $\gamma$ with $T Y \leq \gamma$.

Proof. Write $H$ for $T$ considered as a mere endofunctor, write $T^{\prime}$ for the pointed endofunctor $1+H$, and observe that the map $\alpha: T^{\prime} \rightarrow T$ of endofunctors given by $\alpha=\langle\tau, 1\rangle: 1+H \rightarrow T$ is a retraction. The result now follows from Theorem 16.11 in the light of Theorem 21.1.

\section{FREE MONADS AND FREE MONOIDS}

\section{Free and algebraically-free monads}

22.1. That reliance on the context, which has enabled us to manage without a notational distinction between a mere endofunctor $H$ and a pointed endofunctor $T=(T, \tau)$, becomes too stretched when we pass to a third level and consider a monad $\left(P: A \rightarrow A, \pi: 1 \rightarrow P, \nu: P^{2} \rightarrow P\right)$. Accordingly we denote the monad $(P, \pi, \nu)$ by the bold-face letter $P$, but use the ordinary letter $P$ for the pointed endofunctor $(P, \pi)$ whenever confusion is unlikely. Then $\mathrm{P}-\mathrm{Alg}$ is the full subcategory of $P$-Alg determined by those $(A, a)$ where the action a satisfies, in addition to the unit condition $a \cdot \pi A=1$, also the associativity condition $a \cdot P a=a \cdot v A$.

If $\mathbf{P}$ is such a monad, $T=(T, \tau)$ is a pointed endofunctor, and $\alpha:(T, \tau) \rightarrow(P, \pi)$ is a map of pointed endofunctors, we write $\alpha^{t}$ for the restriction

$$
\text { P-Alg } \rightarrow \text { P-Alg } \underset{\alpha^{\#}}{\longrightarrow} T-A l g
$$

of $\alpha^{\# \prime}$; observe that $\alpha^{\dagger}$ commutes with the forgetful functors P-Alg $\rightarrow A$ and $T-A l g \rightarrow A$. Proposition 5.2 of Barr [2] has the following simple extension to the pointed case:

PROPOSITION 22.1. Every functor P-Alg $\rightarrow$ T-Alg commuting with the forgetful functors is $\alpha^{\dagger}$ for a unique $\alpha$, namely the composite 


$$
T \underset{T \pi}{\longrightarrow} T P \underset{p}{\longrightarrow} P
$$

where $(P A, p A)$ is the image of the free P-algebra (PA, $V A)$. Moreover, if $(T, \tau)$ underzies a monad $T=(T, \tau, \mu)$, then $\alpha^{\dagger}$ takes its values in T-Alg $\subset T$-Alg if and only if $\alpha$ is a monad-map $T \rightarrow P$.

We say that the monad $P$, with the pointed-endofunctor map $\alpha: T \rightarrow P$, is the algebraically-free monad on $T$ if $\alpha^{\dagger}$ is an isomorphism of categories; that is, if every T-action $a: T A \rightarrow A$ is $\bar{a} \cdot \alpha A$ for a unique P-action $\bar{a}$, and every $T$-algebra map $f: A \rightarrow A^{\prime}$ is also a P-algebra-map. The phrase is meant to suggest that $P$ is the "free monad-with-the-same-algebras"; its adverbial form is justified by the following consequence of Proposition 22.1:

PROPOSITION 22.2. If the algebraically-free-monad on the pointed endofunctor $T$ exists, it is the free monad, in the ordinary sense, on this pointed endofunctor.

The observation of $\$ 5.1$, that the forgetful functor $T-A l g \rightarrow A$ is monadic whenever it has a left adjoint, gives:

THEOREM 22.3. The algebraically-free monad on $T$ exists if and only if the forgetful functor $U: T-A l g \rightarrow A$ has a left adjoint $F ;$ and then it is the monad $P$ arising from the adjunction $F \rightarrow U$. In particular it exists if $T$ satisfies the hypotheses of Theorem 15.6 or Theorem 21.6.

In the case where $T=1+H$ for a mere endofunctor $H$, we speak of the algebraically-free monad on $H$; which by Proposition 22.2 is also the free monad, in the ordinary sense, on the endofunctor $H$ : since $T=I+H$ is clearly the free pointed-endofunctor on $H$.

22.2. Barr gives an example in [3], $\$ 5$, for an $A$ that is neither complete nor cocomplete, where the free monad on an endofunctor $H$ exists without being the algebraically-free-monad.

Observe that, for any monad $P=(P, \pi, v)$, we have a monad-map $\pi: 1 \rightarrow P$ from the unit-monad 1 . If $A$ is a one-object category that is a group, every map is an isomorphism, and hence every monad is isomorphic, to 1 . An endomorphism of the mere endofunctor $I=I_{A}$ is an element in the centre of the group $A$; if this centre is trivial, the category of monads on $A$ is equivalent to the unit category, and hence the 
endofunctor $H=1$ is 1 .

An object of $1-A l g$ is an element $x$ of the group $A$, and a morphism $x \rightarrow y$ is an element $z$ with $z^{-1} x z=y$; there are no such morphisms if $x$ is the unit element of $A$ and $y$ is not. However $\mathrm{I}-\mathrm{Alg}=\mathrm{A}$; so that in general $\mathrm{l}-\mathrm{Alg}$ is not equivalent to $1-\mathrm{Alg}$.

22.3. Yet the converse of Proposition 22.2 does hold if $A$ is complete and has small hom-sets : this is Corollary 5.10 of Barr [2]; but his proof, in its appeal to his Proposition 5.6 with its use of the special adjoint functor theorem, seems to be incomplete unless $A$ is wellpowered.

PROPOSITION 22.4. If $\mathrm{A}$ is complete (but not necessarily cocomplete) and has small hom-sets, and if the free monad ( $P, \alpha: T \rightarrow P$ ) on the pointed endofunctor $T$ exists, it is the algebraically-free monad; and similarly when $T$ is replaced by a mere endofunctor $H$.

Proof. The actions on $A$, and on the arrow-category $A^{2}$, of the . strict monoidal category End $A$ of endomorphisms of $A$, both admit right adjoints; in that we have, for any endomorphism $H$ of $\mathrm{A}$,

$$
\begin{aligned}
& A(H A, B) \cong(\text { End } A)(H,\{A, B\}), \\
& A^{2}(H f, g) \cong(\text { End } A)(H,(f, g)),
\end{aligned}
$$

where $\{A, B\}$ is the right Kan extension of $B: 1 \rightarrow A$ along $A: I \rightarrow A$, given by

$$
\{A, B\} C=B^{A(C, A)} \text {, }
$$

and where $\langle f, g\rangle$ is defined by the pullback

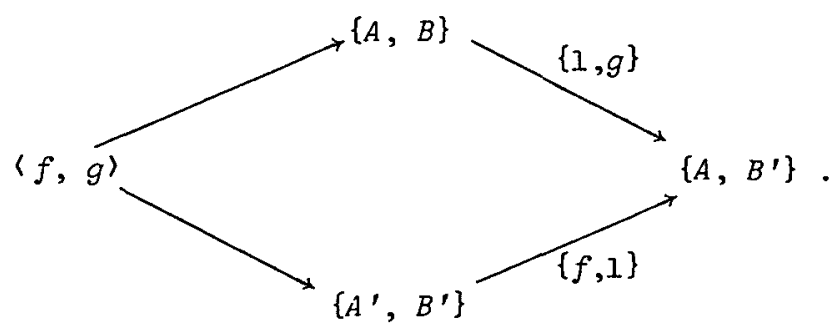

By the general principles of such actions with right adjoints, $\{A, A\}$ and $\langle f, f\rangle$ have canonical monad-structures. Pointed-endofunctor maps 
$T \rightarrow\{A, A\}$ and monad-maps $P \rightarrow\{A, A\}$ correspond respectively to $T$-actions $T A \rightarrow A$ and $P$-actions $P A \rightarrow A$, while pointed-endofunctor maps $T \rightarrow\langle f, f\rangle$ and monad-maps $P \rightarrow\langle f, f\rangle$ correspond to morphisms of the respective algebras. It follows at once that $\alpha^{\dagger}$ is an isomorphism if $(P, a)$ is the free monad on the pointed $T$; and similarly of course in the unpointed case.

\section{Free and algebraically-free monoids}

23.1. A monad $P$ on $A$ is a monoid in the monoidal category End $A$, the tensor product in which is composition; and conversely a monoid $P$ in any monoidal category $A$ gives a monad $P \otimes-$ on $A$. So each of monads and monoids can be discussed in terms of the other; we in fact find it convenient to refer monoids back to monads.

Suppose then that $A$ is a monoidal category - not in general symmetric - with tensor product $\otimes$ and unit object $I$. We change notation to the extent that $H$ (respectively $T=(T, \tau)$, $\mathbf{P}=(P, \pi, \nu)$ ) represents an object (respectively pointed object, monoid) in A rather than the corresponding endofunctor $H \otimes-$ (respectively pointed endofunctor $T \otimes-$, monad $P \otimes-$ ). By an action of $H$ on $A$ we now mean an action of $H \otimes-$, and we write $H-A l g$ for $(H \otimes-)-A l g$; similarly for $T-A l g$ and P-Alg. Observe that if $a: T \otimes A \rightarrow A$ is an action of $T$ on $A$, then $a \otimes B: T \otimes A \otimes B \rightarrow A \otimes B$ is an action of $T$ on $A \otimes B$, so that $-\otimes B$ gives a functor $T-A l g \rightarrow T-A l g$; and similarly. for actions of $H$ or $P$. Given a map $\alpha:(T, \tau) \rightarrow(P, \pi)$ of pointed objects we write $\alpha^{\dagger}: P_{-A l g} \rightarrow T-A l g$ for the $(\alpha \otimes-)^{\dagger}$ of $\S 22.1$; observe that $\alpha^{\dagger}((A, \bar{a}) \otimes B)=\alpha^{\dagger}(A, \bar{a}) \otimes B$.

We say that the monoid $\mathbf{P}$, with the pointed-object map $\alpha: T \rightarrow P$, is the algebraically-free monoid on $T$ if $\alpha^{\dagger}: \mathrm{P}-\mathrm{Alg} \rightarrow T-\mathrm{Alg}$ is an isomorphism of categories; that is, if every T-action $a: T \otimes A \rightarrow A$ is $\bar{a} \cdot(\alpha \otimes A)$ for a unique $P$-action $\bar{a}$, and every $T$-algebra map is also a $P$-algebra map; in other words, if $\alpha \otimes-: T \otimes-\rightarrow P \otimes-$ gives the algebraically-free monad on $T \otimes-$. Similarly when $T$ is replaced by a mere object $H$; the algebraically-free monoid on $H$ is the algebraically-free monoid on the pointed $I+H$. The uniqueness of the 
algebraically-free monoid on $T$, when it exists, can be deduced from the observations above, or directly from the last assertion of the following; which is stated for the pointed case but applies equally to the unpointed one:

THEOREM 23.1. The algebraically-free monoid on $T$ exists if and only if there is a T-algebra $(P, p)$ and a map $\pi: I \rightarrow P$ such that, for each $A \in A, P \otimes A$ with the unit $\pi \otimes A: A \rightarrow P \otimes A$ is the free T-algebra on $A$. When it does exist, $\mathrm{P}$ is the free monoid on $T$, with unit $\alpha: T \rightarrow P$ the composite

$$
T \underset{T \otimes \pi}{\longrightarrow} T \otimes P \underset{p}{\longrightarrow} P
$$

Proof. First, let $\alpha: T \rightarrow P$ give the algebraically-free monoid on $T$. Then, since the free P-algebra on $A$ is $(P \otimes A, \nu \otimes A)$ with the unit $\pi \otimes A$, the free $T$-algebra on $A$ is $(P \otimes A, p \otimes A)$ with the same unit; where $p=v \cdot(\alpha \otimes P): T \otimes P \rightarrow P$.

Next, suppose that the forgetful functor $U: T-A l g \rightarrow A$ has a left adjoint $F$ sending $A$ to $P \otimes A$, for some $T$-algebra $(P, p)$, with unit of the form $\pi \otimes A: A \rightarrow P \otimes A$. Then, since $\pi \otimes P$ is the unit of the free $T$-algebra on $P$, there is a unique map $\nu: P \otimes P \rightarrow P$ of T-algebras with $\nu \cdot(\pi \otimes P)=1$; whence moreover $\nu \otimes A: P \otimes P \otimes A \rightarrow P \otimes A$ must be the unique map of $T$-algebras with $(\nu \otimes A) \cdot(\pi \otimes P \otimes A)=1$. Hence $\nu \otimes-$ is the multiplication of the monad $U F$ on $A$, which therefore has the form $(P \otimes-, \pi \otimes-, \nu \otimes-)$ and thus arises from a monoid $P=(P, \pi, \nu)$ in $A$. The isomorphism $P-A l g \rightarrow T-A l g$, whose existence follows from Theorem 22.3 , is now by Proposition 22.1 given by $\alpha^{\dagger}=(\alpha \otimes I)^{\dagger}$, where $\alpha$ is defined by (23.1).

Lastly, suppose $\alpha^{\dagger}$ to be an isomorphism as above, let $\mathrm{R}=(R, \rho, \lambda)$ be any monoid and $B: T \rightarrow R$ a map of pointed objects. Then, by Proposition 22.1, $B^{\dagger}: \mathrm{R}-\mathrm{Alg} \rightarrow T-\mathrm{Alg}$ is $\alpha^{\dagger} \delta^{\dagger}$ for a unique monad-map $\delta: P \otimes-\rightarrow R \otimes-$. For a $\mathrm{P}$-algebra $A$ and an object $B$ we have $\delta^{\dagger}(A \otimes B)=\left(\delta^{\dagger} A\right) \otimes B$, since the corresponding thing is true for $B^{\dagger}$ and the isomorphism $\alpha^{\dagger}$. It follows that $\delta^{\dagger}$ has the form $\gamma^{\dagger}=(\gamma \otimes I)^{\dagger}$ for some $\gamma: P \rightarrow R$, namely $\gamma=\delta I$; then that $\gamma$ is a monoid-map $P \rightarrow R$; and finally that it is the unique one with $\gamma \alpha=\beta$. 
23.2. Unless the monoidal structure has special properties, the existence of the algebraically-free monoid on $T$ is a strong condition, even for very good categories $A$; it does not follow from the existence of the free monoid on $T$, nor from the existence of the algebraically-free monad on $T \otimes-$ : the latter may well exist without being of the form $P \otimes-$ with a unit of the form $\alpha \otimes-$.

Consider for example the monoidal structure on $A=$ Set having as tensor product the coproduct + , for which the unit object is the empty set 0 . For any monoid $P=(P, \pi, \nu)$, the unit $\pi: 0 \rightarrow P$ is the unique map, while the left and right unit-axioms force $\nu: P+P \rightarrow P$ to be the codiagonal. Hence the category of monoids in $A$ is $A$ itself, and the free monoid on the (mere) object 0 is 0 . Yet the functor $0+-: A \rightarrow A$ is the identity, and the algebraically-free monad on this is $N \times$ - where $N$ is the $x$-monoid of the natural numbers under addition. This monad is quite different from the monad $0+-$ given by the free + -monoid 0 on 0 .

However it is otherwise when the monoidal structure on $A$ is rightclosed, in the sense that $A(A \otimes B, C)$ is isomorphic, naturally in $A$, to $A(A,[B, C])$ for some $[B, C]$.

PROPOSITION 23.2. If $\mathrm{A}$ is right-closed, the algebraically-free monoid on $T$ exists if and only if the free $T$-algebra on $I$ exists. If moreover A has pulzbacks, the free monoid on $T$, if it exists, is algebraically free.

Proof. Let $(P, p)$ be the free $T$-algebra on $I$, with unit $\pi: I \rightarrow P$. For any $T$-algebra $(A, a)$ and any object $B$ let $a_{B}: T \otimes[B, A] \rightarrow[B, A]$ be the map corresponding under the adjunction to $a \cdot(1 \otimes e): T \otimes[B, A] \otimes B \rightarrow A$, where $e:[B, A] \otimes B \rightarrow A$ is the evaluation. Then the composite of $a_{B}$ with $\tau \otimes I:[B, A] \rightarrow T \otimes[B, A]$ corresponds under the adjunction to $a \cdot(1 \otimes e) \cdot(\tau \otimes 1)=a \cdot(\tau \otimes 1) \cdot(1 \otimes e)$, which is $e$ since $a \cdot(\tau \otimes 1)=1$ and we are notationally suppressing isomorphisms $I \otimes X \cong X$. Thus $a_{B} \cdot(\tau \otimes 1)=1$, and $a_{B}$ is a $T$-action on $[B, A]$.

It is immediate that $f: P \otimes B \rightarrow A$ is a $T$-algebra map if and only if the corresponding $\bar{f}: P \rightarrow[B, A]$ is; since $\bar{f} \pi$ corresponds to 
$f \cdot(\pi \otimes B)$, it follows that $\pi \otimes B: B \rightarrow P \otimes B$ is the free $T$-algebra on $B$; and we now appeal to Theorem 23.1.

For the final assertion, we define $\langle f, g\rangle$ as in (22.1), but with $[A, B]$ in place of $\{A, B\}$, and imitate the proof of Proposition 22.4.

23.3. In some cases the slightly weaker condition that $-\otimes A$ preserves colimits can replace right-closedness in the first part of Proposition 23.2.

THEOREM 23.3. If each $-\otimes A: A \rightarrow A$ preserves colimits, the algebraically-free monoid on $T$ exists if the free T-algebra on $I$ exists constructively. In particular it exists if, for some regular $\alpha$, $T \otimes-: A \rightarrow A$ preserves the E-tightness of $\left(M^{\prime}, \alpha\right)$-cones, where $A$ is $E$ - and E'-cowezipowered.

Proof. If $X$ is the free-algebra sequence for $I$, it is clear from the construction of $X$ in $\$ 17.2$ that $X \otimes A$ is the free-algebra sequence for $A$. If the first converges to $(P, p)$, the second converges to $(P \otimes A, P \otimes A)$; and we appeal again to Theorem 23.1.

In these circumstances; we may say that the free monoid on $T$ exists constructively, being given by the convergence of $X$. The explicit form of $X$ is simplest when $T=I+H$ for a mere object $H$; then Theorem 20.4 gives

$$
X_{0}=I, \quad X_{\beta+1}=I+H \otimes X_{\beta}, \quad X_{\alpha}=\underset{\beta<\alpha}{\operatorname{colim}} X_{\beta} .
$$

If the sequence converges at $\alpha$, so that $X_{\alpha} \cong X_{\alpha+1}=I+H \otimes X_{\alpha}$, then $P$ is $X_{\alpha}, \pi$ is the injection $I \rightarrow X_{\alpha}, v: X_{\alpha} \otimes X_{\alpha} \rightarrow X_{\alpha}$ is the colimit of the maps $X_{\beta} \otimes X_{\alpha} \rightarrow X_{\alpha}$ defined by induction on $\beta$ using the injection $H \otimes X_{\alpha} \rightarrow X_{\alpha}$, and the map $H \rightarrow P$ is the composite of the latter injection with. $\perp \otimes \pi: H \rightarrow H \otimes X_{\alpha}$.

Of course the formula (23.2) simplifies in the classical case where both $-\otimes A$ and $A \otimes$ - preserve colimits, as when $A$ is biclosed. In fact we then only need preservation of coproducts, not all colimits: as we have when $A$ is Top and $\otimes$ is $x$. For we have the classical result whose proof is immediate: 
THEOREM 23.4. When $-\otimes A$ and $A \otimes$ - preserve countable coproducts, the algebraically-free monoid on a (mere) object $H$ is $P=\sum_{n \in \mathbb{N}} H^{(n)}$, where $H^{(n)}=H \otimes H \otimes \ldots \otimes H$.

REMARK. Free monoids, as distinct from free monads, in cases where Theorem 23.4 does not apply, were first considered by Dubuc [6], under the hypothesis that $T \otimes$ - or $H \otimes$ - has some rank.

\section{THE CATEGORY OF ALGEBRAS FOR A MONAD}

\section{Reduction to the well-pointed case}

24.1. Now let $T=(T, \tau, \mu)$ be a monad on $A$, and write $T$ for the pointed endofunctor $(T, \tau)$; so that we have full inclusions

$$
\text { T-Alg } \subset T-A l g \subset T / A \text {. }
$$

Given $(A, a, B)$ in $T / A$ consider the pushout

$(24.1)$

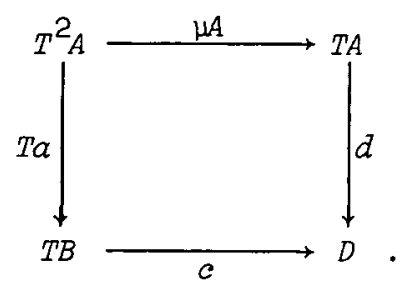

Since $\mu A \cdot T \tau A=\mu A \cdot \tau T A=1$, the map $\mu A$ is the coequalizer of $T \tau A \cdot \mu A$ and 1 ; or equally of $\tau T A \cdot \mu A$ and 1 . Thus $c$ is the coequalizer of the maps $T a \cdot T \tau A \cdot \mu A$ and $T a$ in

$(24.2)$

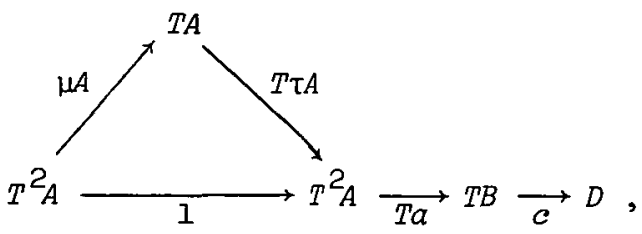

or equally of the maps we get if we replace $T \tau A$ here by $\tau T A$. Moreover, composing (24.1) with $T \tau A$ shows that

$$
d=c \cdot T a \cdot T \tau A \text {. }
$$


Now define an endofunctor $L$ of $T / A$ by setting $(24.4)$

$$
L(A, a, B)=(B, C, D),
$$

and making $L$ functorial in the obvious way. We compare $L$ with the endofunctor $S$ of $\$ 17.1$, using the notation of (17.1). Since $\mu A \cdot \tau T A=\mu A \cdot T \tau A$ it follows from $(24.1)$ that $c \cdot T a \cdot \tau T A=c \cdot T a \cdot T \tau A$, so that $c$ factorizes through the $b$ of (17.1) as $c=q b$ for a unique $q$; and moreover $q$ is a regular epimorphism, since both $b$ and $c$ are. We therefore have an epimorphism

$$
(1, q):(B, b, C) \rightarrow(B, c, D)
$$

in $T / A$, which is clearly the $(A, a, B)$-component of an epimorphic natural transformation

$$
\phi: S \rightarrow L
$$

If we now define $\lambda: 1 \rightarrow L$ by $\lambda=\phi \sigma$, it follows from Proposition 7.1 that $(L, \lambda)$ is again a well-pointed endofunctor on $T / A$. In fact we have:

THEOREM 24.1. L-Alg is the repletion of T-Alg .

Proof. By Proposition 7.1, an L-algebra $(A, a, B)$ is an $S$-algebra for which $\phi(A, a, B)$ is an isomorphism; that is, for which $q$ is an isomorphism, or equivalently for which the coequalizer $b$ in (17.1) already equalizes the two maps $T^{2} A \rightarrow T B$ in (24.2). But by Theorem 14.4, an $S$-algebra, to within isomorphism, is a $T$-algebra $(A, a)$; and for this we have $b=a$. The requirement that $a$ equalize the two maps $T^{2} A \rightarrow T B=T A$ in (24.2) is, because $a \cdot \tau A=1$ for a $T$-algebra, exactly the associativity axiom for a $\operatorname{T-algebra}(A, a)$.

24.2. We can now form the free-L-algebra sequence $\hat{L}(A, a, B)$ in $T / A$, and say that $(A, a, B)$ admits a constructive reflexion in T-Alg if this converges. We can further give $\hat{L}(A, a, B)$ explicitly. We define a sequence $X: \infty \rightarrow A$ and a natural transformation $x: T X \rightarrow X^{+}$by (17.2)-(17.6), except that we replace $(17.4)$ by 
$(24.7)$

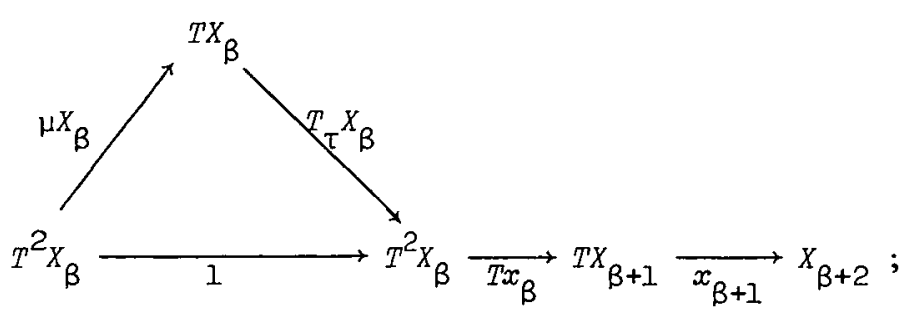

then, corresponding to Theorem 17.3, and with the same proof, we have:

THEOREM 24.2. $\hat{L}(A, a, B)$ is $\left(X, x, X^{+}\right)$, and converges at $\alpha$ exactly when $X$ does. Then the reflexion of $(A, a, B)$ in T-Alg is $X_{\alpha}$ with the action $x_{\alpha}: T X_{\alpha} \rightarrow X_{\alpha+1}=X_{\alpha}$.

We may call $X$ the T-algebra reflexion sequence for $(A, \alpha, B)$. It is at once seen to coincide with the sequence of Koubek and Reiterman [13], $\$ 10.3$, modulo the difference between our T/A and their GPA.

\section{Existence theorems for the reflexion of $T / A$ into $T-A 1 g$}

25.1. An analysis of the argument used in the proof of Proposition 15.5 gives:

LEMMA 25.1. Consider an $\alpha$-chain in T/A given by $X, Y: \alpha \rightarrow A$ and $x: T X \rightarrow Y$, and a cone $\left(t_{\beta}, s_{\beta}\right):\left(X_{B}, x_{B}, Y_{\beta}\right) \rightarrow\left(A^{\prime}, a^{\prime}, B^{\prime}\right)$ over it. If the cone $\left(t_{\beta}, s_{\beta}\right)$ in $T / A$ is E-tight, so is the cone $\left(t_{\beta}\right)$ in $A$. If the cones $\left(t_{\beta}\right)$ and $\left(T t_{\beta}\right)$ in $A$ are both E-tight, the cone $\left(t_{\beta}, s_{\beta}\right)$ in T/A is E-tight if and only if the cone $\left(s_{\beta}\right)$ in $A$ is so.

THEOREM 25.2. Let $T$ preserve, for some regular $\alpha$, the E-tightness of $\left(M^{\prime}, \alpha\right)$-cones. Then if $A$ is $E$ - and $E^{\prime}$-cowellpowered, T-Alg is constructively reflective in T/A. Even without the cowellpoweredness, it is stizl reflective if $M^{\prime}=M$ and $(E, M)$ is proper.

Proof. We deal first with the latter assertion, which is easier. By Proposition 15.5, $S$ preserves the E-tightness of $(M, \alpha)$-cones since $T$ does; so then does $L$ by $\$ 2.4$ since $\phi: S \rightarrow L$ lies in $E$ (for the regular epimorphism $q$ of (24.5) lies in any proper $E$ ); and the result follows by Theorem 7.5 . 
Turning to the first assertion, we apply Proposition 4.2 with Remark 4.3 to $T$, and to the sequence $X$ of Theorem 24.2 , to obtain a limitordinal $\delta$ such that, for each $\gamma \geq \delta$, if the cone $\left(X_{B}^{\gamma}\right)_{B<\delta}$ is $E$-tight, so are the cones $\left(T X_{\beta}^{\gamma}\right)_{\beta<\delta}$ and $\left(T^{2} X_{\beta}^{\gamma}\right)_{\beta<\delta}$. We then conclude the proof by applying Proposition 6.1, with $S$ and $\alpha$ replaced by $L$ and $\delta$; so it remains to show that $L$ and $\delta$ satisfy the hypotheses of Proposition 6.1. Writing $\hat{L}$ for $\hat{L}(A, a, B)=\left(X, x, X^{+}\right)$and "tight" for "E-tight", suppose then that $\left(\hat{L}_{B}^{\gamma}\right)_{B<\delta}$ is tight. By Lemma 25.1, $\left(X X_{B}^{\gamma}\right)_{B<\delta}$ is tight; then, by our choice of $\delta,\left(T X_{\beta}^{\gamma}\right)_{\beta<\delta}$ is tight; so that by Lemma 25.1 again, $\left(X_{B+1}^{\gamma+1}\right)_{\beta<\delta}$ is tight; and by the choice of $\delta$ again, $\left(T X_{B+1}^{\gamma+1}\right)_{B<\delta}$ is tight. Moreover, by our choice of $\delta,\left(T^{2} X_{B}^{\gamma}\right)_{B<\delta}$ is tight.

Now $X_{\beta+2}$ is given by the coequalizer $(24.7)$, and $X_{\gamma+2}$ by a similar one. Hence $X_{\beta+2}^{\gamma+2}$ is the colimit of a natural transformation between these diagrams, whose various components are $T^{2} X_{\beta}^{\gamma}$, $T X_{\beta}^{\gamma}$, and $T X_{\beta+1}^{\gamma+1}$. Thus by $\S 2.4$ the cone $\left(X_{\beta+2}^{\gamma+2}\right)_{\beta<\delta}$, as a colimit of tight cones, is tight. Since, by $(24.4)$ and $(24.7), \underset{L}{L} \hat{L}=\left(X^{+}, x^{+}, X^{++}\right)$, it now follows from Lemma 25.1 that $\left(L \hat{L}_{\beta}^{\gamma}\right)_{B<\delta}$ is tight, as required.

REMARK 25.3. The author does not see how to prove the reflectivity without $E^{\prime}$-cowellpoweredness when $E$ is the isomorphisms - contrast Theorem 15.6. The first part of Theorem 25.2 is essentially Theorem 10.5 of Koubek and Reiterman [13].

As in $\$ 14.1$, we have as an immediate corollary:

THEOREM 25.4. Under the hypotheses of Theorem 25.2, T-Alg is cocomplete. Moreover any $\alpha^{\dagger}: \mathrm{T}-\mathrm{Alg} \rightarrow \mathrm{T}^{\prime}-\mathrm{Alg}$ induced by a monad-map $\alpha: T^{\prime} \rightarrow T$ has a left adjoint. 
The cocompleteness of T-Alg was proved by Schubert [16] when $T$ preserves the colimits of $\alpha$-chains. Barr deals in Theorem 3.3 of [2] with the case where $T$ preserves the colimits of $(M, \alpha)$-chains; but his proof seems faulty to the present author, who sees no easy way to set it right.

25.2. The results of T-Alg are especially simple in the special case where $T E \subset E$. Of course the free T-algebra on $B$ always exists, being $(T B, \mu B)$. But in fact it exists constructively; for when, in accordance with Proposition 14.3, we apply $\hat{L}$ to $(B, 1, T B)$ to get $\left(X, x, X^{+}\right)$as in Theorem 24.2, $X$ converges already at $\alpha=1$; since $x_{1}$, as the coequalizer of $T \tau B \cdot \mu B$ and 1 , is $\mu B$.

We can now imitate the argument of Theorem 16.5. $(A, a, B)$ has a reflexion (respectively, constructive reflexion) into T-Alg if $L(A, a, B)$ does. But, by $(24.4), L(A, a, B)=(B, c, D)$, where $c$ from (24.2) is a regular epimorphism and hence in the $E$ of any proper $(E, M)$; so that $(1, C):(B, I, T B) \rightarrow(B, C, D)$ is in $E$. Moreover $L E \subset E$, since $S E \subset E$ by Proposition 16.1 and since $\phi: S \rightarrow L$ is in $E$ (as we saw in the proof of Theorem 25.2). Hence Proposition 8.3 gives:

THEOREM 25.5. Let $T E \subset E$ where $(E, M)$ is proper. Then T-AIg is reflective in $T / A$, and constmuctively so when $A$ is E-cowellpowered. Consequently T-Alg is cocomplete, and algebraic functors $\alpha^{\dagger}: \mathrm{T}_{-\mathrm{Alg}} \rightarrow \mathrm{T}^{\prime}-\mathrm{Alg}$ have left adjoints.

\section{COLIMITS OF MONADS AND MONOIDS}

\section{Algebraic colimits of monads}

Denote by Monad $A$ the category of monads on $A$ and monad-maps, and consider a functor $T: K \rightarrow$ Monad $A$ with $K$ small; writing $T_{k}=\left(T_{k}, \tau_{k}, \mu_{k}\right)$ for the value of $T$ at the object $k$, and $T_{\phi}$ for its value at the map $\phi: k \rightarrow k^{\prime}$.

Even when $A=$ Set,$T$ may admit no colimit; as is shown by Proposition 6.10 of Barr [2]. What he does show, in his Theorem 6.6, is the cocompleteness of the full subcategory of Monad Set given by the monads with rank. However, as Blackwell pointed out in his thesis [4], 
this does not suffice for practical applications; one wants a (colim T) algebra to be an $A$ with compatible $T_{k}$-actions, and for this colim $T$ must be the colimit in the whole category Monad A. Blackwell [4] established the existence of the colimit in this "algebra-related" sense when each $T_{k}$ has a rank and $A$ is complete as well as cocomplete, with small hom-sets. We now take this somewhat further.

We define a T-algebra to be an object $A \in A$ together with, for each $k \in K$, a $T_{k}$-action $a_{k}: T_{k} A \rightarrow A$, subject to the compatibility requirements $a_{k^{\prime}} \cdot T_{\phi}=a_{k}$ for $\phi: k \rightarrow k^{\prime}$. A map $f: A \rightarrow A^{\prime}$ of T-algebras is to be a map of $T_{k}$-algebras for each $k$. In other words, $\mathrm{T}-\mathrm{Alg}$ is the limit of the functor $K \rightarrow$ Cat sending $k$ to $\mathrm{T}_{k}-\mathrm{Alg}$ and sending $\phi$ to $T_{\phi}^{\dagger}$; we may write $Q_{k}:$ T-Alg $\rightarrow T_{k}-$ Alg for the projection functors.

Any cone $r=\left(r_{k}: T_{k} \rightarrow P\right)$ over $T$ in Monad $A$ induces a functor $r^{\dagger}:$ P-Alg $\rightarrow$ T-Alg which commutes with the forgetful functors to A; namely the functor with components $Q_{k} r^{\dagger}=r_{k}^{\dagger}$. It is moreover immediate from Proposition 22.1 that:

PROPOSITION 26.1. Every functor P-Alg $\rightarrow$ T-Alg commuting with the underlying functors is $r^{\dagger}$ for a unique cone $r$ as above.

We now say that the monad $P$, with the cone $r$, is the algebraic colimit of $T: K \rightarrow$ Monad $A$ if $r^{\dagger}$ is an isomorphism of categories. From Propositions 26.1 and 22.1 we conclude that

PROPOSITION 26.2. When the algebraic colimit of $T$ exists, it is the colimit in the ordinary sense of $T: K \rightarrow$ Monad A.

The proof of Proposition 22.4 adapts at once to give:

PROPOSITION 26.3. If $\mathrm{A}$ is complete and has small hom-sets, the colimit of $T$ is algebraic whenever it exists.

It is immediate that the forgetful functor $U: T-A l g \rightarrow A$ creates limits and U-absolute colimits; whence 
PROPOSITION 26.4. The algebraic colimit of $T$ exists if and only if the forgetful functor $U: \mathrm{T}_{-\mathrm{Al}} \rightarrow \mathrm{A}$ has a left adjoint $F$; and then it is the monad $P$ arising from the adjunction $F \rightarrow U$.

\section{An existence theorem for algebraic colimits of monads}

27.1. Given $T: K \rightarrow$ Monad $A$ as above, we do not change $T$-Alg if we extend $K$ and $T$ by adding to $K$ a new initial object 0 , by setting $T_{0}$ equal to the identity monad 1 , and by taking $T_{0} \rightarrow T_{k}$ to be the monad-map $\tau: 1 \rightarrow T_{K}$. We suppose this done, and now use $K$ and $T$ in this new meaning.

Write $T$, with $r_{k}: T_{k} \rightarrow T$, for the colimit of $T$ seen as a functor $K \rightarrow$ End $A$. Then $T$ is a pointed endofunctor $(T, \tau)$, where $\tau=r_{0}: 1 \rightarrow T$, and the $r_{k}$ are pointed-endofunctor maps. We write as usual $T-A l g$ for $(T, \tau)-A l g$, and similarly $T_{k}-\mathrm{Alg}$ for $\left(T_{k}, \tau_{k}\right)-\mathrm{Alg}$. To give a map $a: T A \rightarrow A$ is just to give component-maps $a_{k}=a r_{k}: T_{k} A \rightarrow A$ for each $k$, subject to the compatibility condition $a_{k}=a_{k} \cdot T_{\phi} A$; and the unit-axiom $a \cdot \tau_{A}=1$ for $a$ is just $a_{0}=1$, and hence equivalent to $\alpha_{k} \cdot \tau_{k} A=1$ for each $k$. It follows that $\mathrm{T}-\mathrm{Alg}$ is the full subcategory of $T$-Alg given by those $(A, a)$ such that, for each $k$, the $T_{k}$-algebra $r_{k}^{\#}(A, a)=\left(A, a_{k}\right)$ is in fact a $T_{k}$-algebra. We have the full inclusions

$$
T-A l g \subset T-A l g \subset T / A \text {, }
$$

and we shall have shown free $T$-algebras to exist if we prove $T-A l g$ reflective in $T / A$.

Given $(A, a, B)$ in $T / A$ let $c: T B \rightarrow D$ be the joint coequalizer, for all $k \in K$, of the parallel pairs

$(27.1)$

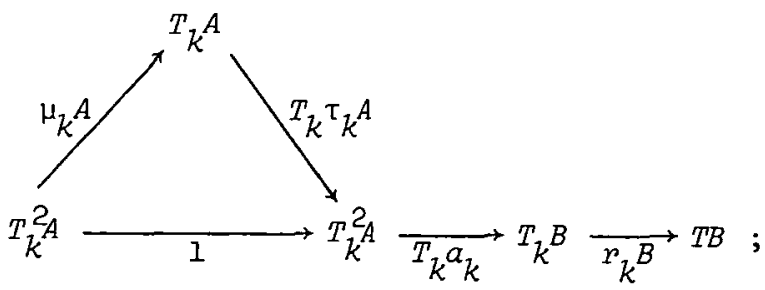


where here too $a_{k}$ stands for $a r_{k}$. Define an endofunctor $L$ of $T / A$ by

$$
L(A, \alpha, B)=(B, C, D),
$$

making it functorial in the obvious way; $c$. $\$ 24.1$. As there, we compare $L$ with the endofunctor $S$ of $\$ 17.1$.

The (three) squares in the diagram

$(27 \cdot 3)$

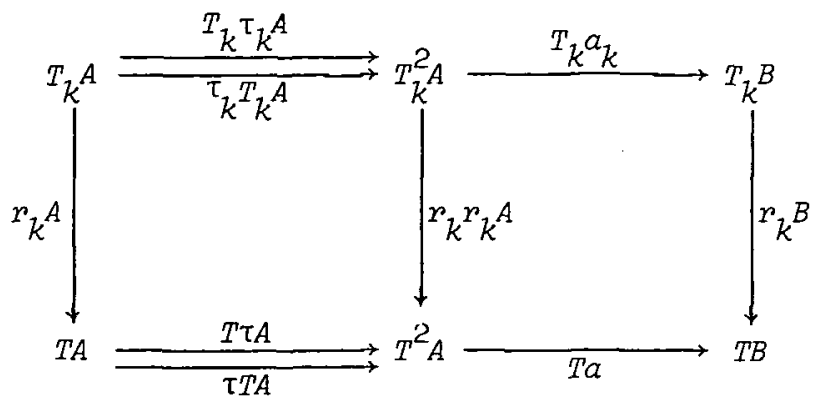

clearly commute. The map $C: T B \rightarrow D$ equalizes the two maps in the top leg of (27.3), this leg being the composite of (27.1) with $\tau_{k} T_{k} A$.

Therefore $c$ equalizes the two maps in the bottom leg; and since the $r_{k} A$ are jointly epimorphic, it equalizes $T a \cdot T \tau A$ and $T a \cdot \tau T A$. It therefore factorizes through the coequalizer $b$ in (17.1) as $c=q b$ for a regular epimorphism.$q$. Thus as in $\$ 24.1$ we have an epimorphic $\phi: S \rightarrow L$ with $(A, a, B)$-component $(1, q) ;$ and $(L, \lambda)$ becomes a well-pointed endofunctor when we set $\lambda=\phi \sigma$.

Arguing as in the proof of Theorem 24.1 - for an S-algebra, that is a $T$-algebra, $B=A$ and $b=a$, and then $\phi$ is an isomorphism if and only if $a$ equalizes all the pairs (27.1), which means exactly that each $a_{k}$ is a $\mathrm{T}_{k}$-action - we have the formally-identical conclusion that $L-\mathrm{Alg}$ is the repletion of T-Alg. We then have Theorem 24.2, except that in the description of $(X, x)$ we replace (24.7) by the analogue using (27.1) in place of $(24.2)$.

THEOREM 27.1. For some regular $\alpha$, let each $T_{k}$ preserve the E-tightness of $\left(M^{\prime}, \alpha\right)$-cones. Then the algebraic colimit of $T$ exists if $A$ is $E_{-}$and $E^{\prime}$-cowellpowered, or if $M^{\prime}=M$ and $(E, M)$ is proper. 
Proof. The second case is dealt with exactly as in the proof of Theorem 25.2; for the first case we modify that proof appropriately.

We begin by observing that the improvement of Proposition 4.1 given in Remark 4.3, to deal with two sequences instead of one, applies equally well to any small set of sequences. By applying it to the sequences $X$ and $T_{k} X$ for $k \in K$, we get under our present hypotheses an improved version of Proposition 4.2: there is a limit-ordinal $\delta$ such that, if $\left(X_{B}^{\gamma}\right)_{B<\delta}$ is tight (=E-tight) for some $\gamma \geq \delta$, so are each $\left\{T_{k} X_{\beta}^{\gamma}\right\}_{\beta<\delta}$ and each $\left(T_{k}^{2} X_{B}^{\gamma}\right)_{B<\delta}$

The proof now proceeds as that of Theorem 25.2, by showing that $L$ and $\delta$ satisfy the hypotheses of Proposition 6.1. From the tightness of $\left(\hat{L}_{\beta}^{\gamma}\right)_{\beta<\delta}$ we get as there that of $\left(X_{B}^{\gamma}\right)_{\beta<\delta}$; hence of $\left(T_{k} X_{\beta}^{\gamma}\right)_{\beta<\delta}$ and of $\left(T_{k}^{2} X_{\beta}^{\gamma}\right)_{\beta<\delta}$; also that of $\left(X_{\beta+1}^{\gamma+1}\right)_{\beta<\delta}$ and hence of $\left(T_{k} X_{\beta+1}^{\gamma+1}\right)_{\beta<\delta}$. We get the tightness of $\left(T X_{B+1}^{\gamma+1}\right)_{\beta<\delta}$ as the colimit of the tight cones $\left(T_{k} X_{B+1}^{\gamma+1}\right)_{\beta<\delta}$; and then the tightness of $\left(X_{\beta+2}^{\gamma+2}\right)_{\beta<\delta}$ as the colimit of tight cones in the (27.1)-analogue of (24.7). This gives the required tightness of $\left(L \hat{L}_{\beta}^{\gamma}\right)_{\beta<\delta}$.

27.2. In some contexts the question arises whether, under the hypotheses of Theorem 27.1, the algebraic colimit $P$ of $T$ inherits the property of preserving the E-tightness of $\left(M^{\prime}, \alpha\right)$-cones. The author does not see how to show this unless $M^{\prime}=$ all maps.

THEOREM 27.2. Let $A$ be E-cowe Ilpowered, and let each $T_{k}$ preserve the E-tightness of all $\alpha$-cones. Then $P$ too preserves the E-tightness of all $\alpha$-cones, where $P$ is the algebraic colimit of $T$.

Proof. If we write (*) for the property of preserving the $E_{-}$ tightness of all $\alpha$-cones, (*) is satisfied by each $T_{k}$ and hence by each 
$T_{k}^{2}$, and also by the colimit $T$ of the $T_{k}$. By Lemma 25.1 therefore, an a-cone in $T / A$ is $E$-tight if and only if each of its components in $A$ is so.

It follows that $L$ satisfies (*). For if $L$ sends a tight a-cone $(s, t)$ to $(u, v)$, we have $u=s$ by (27.2), while $v$ by (27.1) is a colimit of tight cones and hence itself tight.

It then follows inductively that the transfinite powers $L^{\beta}$ of $L$ satisfy $(*)$, and in particular $L^{\infty}$; which is the composite $J R$ of the inclusion $J:$ T-Alg $\rightarrow T / A$ with the reflexion $R: T / A \rightarrow T-A l g$. The forgetful $U: T_{-A l g} \rightarrow A$ is $V J$, where $V: T / A \rightarrow A$ sends $(A, a, B)$ to $A$ and has the left adjoint $G$ sending $C$ to $(C, 1, T C):$ and the left adjoint $F$ of $U$ is $R G$.

By Proposition 26.4, $P=U F=V J R G=V L^{\infty} G$. But $G$ preserves colimits, and sends $E$ to $E$ by $\$ 15.1$, so that $G$ satisfies (*); $L^{\infty}$ satisfies (*) by the above; and $V$ satisfies (*) by Lemma 25.1 . Hence $P$ satisfies $(*)$.

\section{Algebraic colimits of monoids}

Suppose now that $A$ is a monoidal category, and that we change notation as we did in $\S 23$. Denote by Mon $A$ the category of monoids in $A$, and now consider a functor $T: K \rightarrow$ Mon $A$ with $K$ small, sending $K$ to the monoid $T_{k}$. We define a T-algebra to be an $A$ with compatible $T_{k}$-actions $a_{k}: T_{k} \otimes A \rightarrow A ;$ that is, a $(T \otimes-)$-algebra in the sense of §26. Again a cone $\left(r_{k}: T_{k} \rightarrow P\right)$ over $T$, this time in Mon $A$, induces $r^{\dagger}: P_{-A l g} \rightarrow T_{-A l g}$, namely the $(r \otimes I)^{\dagger}$ of $\S 26$.

We say that the monoid $P$, with the cone $r$, is the algebraic colimit of $T$ if $r^{\dagger}$ is an isomorphism of categories; that is, if the monad $P \otimes-$ is the algebraic colimit of $T \otimes-$. By a proof exactly analogous to that of Theorem 23.1, but using Propositions 26.1 and 26.4 in place of Proposition 22.1 and Theorem 22.3, we get:

THEOBEM 28.1. The algebraic colimit of $T$ exists if and only if there is a T-algebra $(P, p)$ and a map $\pi: I \rightarrow P$ such that, for each 
$A \in A, P \otimes A$ with the unit $\pi \otimes A$ is the free T-algebra on $A$. When it does exist, $P$ is the colimit of $\mathbf{T}$, with $r_{k}: T_{k} \rightarrow P$ the composite

$$
T_{k} \underset{T_{k} \otimes \pi}{\longrightarrow} T_{k} \otimes P \underset{p_{k}}{\longrightarrow} P
$$

Then a proof exactly analogous to that of Proposition 23.2 gives:

PROPOSITION 28.2. If $\mathrm{A}$ is right-closed, the algebraic colimit of $T$ exists if and only if the free T-algebra on $I$ exists. If moreover $A$ has pulzbacks, the colimit of $T$, if it exists, is the algebraic colimit.

Finally, as in Theorem 23.3, but appealing now to Theorems 27.1 and 27.2 , we get:

THEOREM 28.3. If each - $\otimes A$ preserves colimits, and if for some reguzar a each $T_{k} \otimes$ - preserves the E-tightness of $\left(M^{\prime}, \alpha\right)$-sones, where $A$ is $E$ - and $E^{\prime}$-cowellpowered, the algebraic colimit $P$ of exists. When $M^{\prime}=$ all maps, $P$ too preserves the E-tightness of $\alpha$-cones.

\section{A comment on polyads}

It is clear that, in their formal aspects, the descriptions of T-Alg in the sense of $\S 26$, of T-Alg for a single monad $T$, of $T-A l g$, and of $H-A . g$, follow a pattern and admit of a common abstract generalization. This was given by Blackwell in his thesis [4].

By a polyad Blackwell means a strict monoidal functor $T$ from a small strict monoidal category $V$ to the strict monoidal category End A; A $T$-aZgebra then consists of an $A \in A$ with compatible actions $a_{x}: T_{x} A \rightarrow A$ for $x \in V$, related to the tensor products (written as juxtaposition) by the requirement that $a_{x y}$ be the composite

$$
T_{x y} A=T_{x} T_{y} A \underset{T_{x} a_{y}}{\longrightarrow} T_{x} A \underset{a_{x}}{\longrightarrow} A
$$

and that $a_{1}: T_{1} A=A \rightarrow A$ be the identity. When $A$ is (not only cocomplete but) complete and with small hom-sets, we may regard the monad $\{A, A\}$ of Proposition 22.4 as a (non-strict) monoidal functor $1 \rightarrow$ End $A$, or again as the composite constant monoidal functor 


$$
V \rightarrow 1 \underset{\{A A\}}{\longrightarrow} \text { End } A \text {. }
$$

Then a T-action on $A$ corresponds to a monoidal natural transformation $T \rightarrow\{A, A\}$, or a "monoidal cone" over $T$ with vertex $\{A, A\} . \mathrm{A}$ universal such mononoidal cone over $T$, with vertex the monad $P$ say what we might call the "monoidal colimit" of $T$ - exists precisely when the forgetful functor $T-A l g \rightarrow A$ has a left adjoint. Blackwell proves its existence, in these circumstances, if each $T_{x}$ has some rank.

In the various situations we have considered, $V$ is in effect presented by generators and relations, while $T$ is given on the generators and subjected to axioms corresponding to the relations. We have given existence theorems under hypotheses weaker than having rank, namely that the generating $T_{x}$ should preserve the E-tightness of $\left(M^{\prime}, \alpha\right)$-cones. It is because this is not inherited by the composite ${ }^{T} x^{T} y$, unless $T_{y} M^{\prime} \subset M^{\prime}$, that we have been forced to more subtle arguments, such as those using the Koubek and Reiterman reduction of $\$ 4$ above. At the same time the individual situations we have considered present various particular features, sometimes allowing stronger results than are available for general polyads, or leading to side-results without meaning in the general case.

Blackwell's thesis goes further, and considers 2-polyads on a 2-category, where new phenomena appear which are of importance in the study of algebraic structures borne by a category. Some of these aspects will be pursued in forthcoming articles by him and by the present author.

\section{References}

[1] Jirí Adámek, "Colimits of algebras revisited", Bull. Austral. Math. Soc. 17 (1977), 433-450.

[2] Michael Barr, "Coequalizers and free triples", Math. Z. 116 (1970), $307-322$.

[3] Michael Barr, "Coequalizers and free triples, II" (Manuscript, McGill University, Montreal, 1979). 
[4] R. Blackwell, "Some existence theorems in the theory of doctrines" (PhD thesis, University of New South Wales, Kensington, 1976).

[5] A.K. Bousfield, "Construction of factorization systems in categories", J. Pure Appl. Algebra 9 (1977), 207-220.

[6] Eduardo J. Dubuc, "Free monoids", J. Algebra 29 (1974), 208-228.

[7] P.J. Freyd and G.M. Kelly, "Categories of continuous functors, I", J. Pure Appl. AZgebra 2 (1972), 169-191; Erratum, J. Pure Appl. Algebra 4 (1974), 121.

[8] Peter Gabriel, Friedrich UImer, Lokal präsentierbare Kategorien (Lecture Notes in Mathematics, 221. Springer-Verlag, Berlin, Heidelberg, New York, 1971).

[9] G. Max Kelly, "Quelques observations sur les démonstrations par récurrence transfinie en algèbre catégorique", Cahiers Topologie Géom. Différentiezze 16 (1975), 259-263.

[10] G.M. Kelly and Ross Street, "Review of the elements of 2-categories", Category Seminar, 75-103 (Proc. Sydney Category Theory Seminar, 1972/1973. Lecture Notes in Mathematics, 420. Springer-Verlag, Berlin, Heidelberg, New York, 1974).

[11] Václav Koubek, "Constructions of continuous functors" (Manuscript, Charles University, Prague, 1978).

[12] Václav Koubek, Jan Reiterman, "Automata and categories - input processes", Mathematical foundations of computer science, 280-286 (4th Symposium, Mariánské Laźnè, 1975. Lecture Notes in Computer Science, 32. Springer-Verlag, Berlin, Heidelberg, New York, 1975).

[13] Václav Koubek and Jan Reiterman, "Categorical constructions of free algebras, colimits, and completions of partial algebras", $J$. Pure App Z. AZgebra 14 (1979), 195-231.

[14] Vĕra Kurková-Pohlová, Václav Koubek, "When a generalized algebraic category is monadic", Comment. Math. Univ. Carolin. 15 (1974), $577-587$.

[15] Jan.Reiterman, "A left adjoint construction related to free triples", J. Pure Appl. Algebra 10 (1977), 57-71. 
[16] Horst Schubert, Categories (translated by Eva Gray. Springer-Verlag, Berlin, Heidelberg, New York, 1972).

[17] Harvey Wolff, "Free monads and the orthogonal subcategory problem", J. Pure Appl. Algebra 13 (1978), 233-242.

Department of Pure Mathematics, University of Sydney, Sydney, New South Wales 2006, Australia. 